ESAIM: M2AN 48 (2014) 553-581

DOI: $10.1051 / \mathrm{m} 2 \mathrm{an} / 2013104$
ESAIM: Mathematical Modelling and Numerical Analysis

www.esaim-m2an.org

\title{
ANALYSIS OF COMPATIBLE DISCRETE OPERATOR SCHEMES FOR ELLIPTIC PROBLEMS ON POLYHEDRAL MESHES
}

\author{
JÉrôme Bonelle ${ }^{1}$ And AleXandre ERn²
}

\begin{abstract}
Compatible schemes localize degrees of freedom according to the physical nature of the underlying fields and operate a clear distinction between topological laws and closure relations. For elliptic problems, the cornerstone in the scheme design is the discrete Hodge operator linking gradients to fluxes by means of a dual mesh, while a structure-preserving discretization is employed for the gradient and divergence operators. The discrete Hodge operator is sparse, symmetric positive definite and is assembled cellwise from local operators. We analyze two schemes depending on whether the potential degrees of freedom are attached to the vertices or to the cells of the primal mesh. We derive new functional analysis results on the discrete gradient that are the counterpart of the Sobolev embeddings. Then, we identify the two design properties of the local discrete Hodge operators yielding optimal discrete energy error estimates. Additionally, we show how these operators can be built from local nonconforming gradient reconstructions using a dual barycentric mesh. In this case, we also prove an optimal $L^{2}$-error estimate for the potential for smooth solutions. Links with existing schemes (finite elements, finite volumes, mimetic finite differences) are discussed. Numerical results are presented on three-dimensional polyhedral meshes.
\end{abstract}

Mathematics Subject Classification. 65N12, 65N08, 65N30.

Received November 12, 2012. Revised July 16, 2013.

Published online January 20, 2014.

\section{INTRODUCTION}

Engineering applications on complex geometries can involve polyhedral meshes with various element shapes. Our goal is to analyze a class of discretization schemes on such meshes for the model elliptic problem

$$
-\operatorname{div}(\underline{\underline{\operatorname{grad}}} p)=s,
$$

posed on a bounded polyhedral domain $\Omega \subset \mathbb{R}^{3}$ with source $s \in L^{2}(\Omega)$. For simplicity, we focus on homogeneous Dirichlet boundary conditions; nonhomogeneous Dirichlet/Neumann boundary conditions for (1.1) can be considered as well. We introduce the gradient and flux of the exact solution such that

$$
\underline{g}=\underline{\operatorname{grad}} p, \quad \underline{\phi}=-\underline{\underline{\lambda}} \underline{\underline{g}}, \quad \operatorname{div} \underline{\phi}=s .
$$

Keywords and phrases. Compatible schemes, mimetic discretization, Hodge operator, error analysis, elliptic problems, polyhedral meshes.

1 EDF R\&D, 6, quai Watier, BP 49, 78401 Chatou cedex, France. jerome.bonelle@edf.fr

2 Université Paris-Est, CERMICS (ENPC), 77455 Marne la Vallée Cedex 2, France. ern@cermics.enpc.fr 
In what follows, $p$ is termed the potential. The conductivity $\underline{\underline{\lambda}}$ can be tensor-valued, and its eigenvalues are uniformly bounded from above and from below away from zero.

Following the seminal ideas of Tonti [33] and Bossavit [6,7], compatible (or mimetic, or structure-preserving) schemes aim at preserving basic properties of the continuous model problem at the discrete level. In such schemes, the localization of the degrees of freedom results from the physical nature of the fields: potentials are measured at points, gradients along lines, fluxes across surfaces, and sources in volumes (in the language of differential geometry, a potential is a (straight) 0-form, a gradient a (straight) 1-form, a flux a (twisted) 2-form, and a source a (twisted) 3-form). Moreover, compatible schemes operate a clear distinction between topological relations (such as $g=\operatorname{grad} p$ and $\operatorname{div} \phi=s$ in (1.2)) and closure relations (such as $\phi=-\underline{\lambda} g$ in (1.2)). The localization of degrees of freedom makes it possible to build discrete differential operators preserving the structural properties of their continuous counterpart. Thus, the only source of error in the scheme stems from the discretization of the closure relation. This step relies on a so-called discrete Hodge operator whose design is the cornerstone of the construction and analysis of the scheme (see, e.g., Tarhassari et al. [32], Hiptmair [26], and more recently Gillette [25]). For the numerical analysis of compatible schemes, we refer to the early work of Dodziuk [18] (extending ideas of Whitney [35]) and Hyman and Scovel [28], and to more recent overview papers by Mattiussi [30], Bochev and Hyman [5], Arnold et al. [3], and Christiansen et al. [12].

Although it is not always made explicit, an important notion in compatible schemes is the concept of orientation; see Bossavit [6], number one, and Kreeft et al. [29]. For instance, measuring a gradient requires to assign an inner orientation to the line (indicating how to circulate along it), while measuring a flux requires to assign an outer orientation to the surface (indicating how to cross it). Discrete differential operators act on entities with the same type of orientation (either inner or outer), while the discrete Hodge operator links entities with a different type of orientation. Therefore, in addition to the primal mesh discretizing the domain $\Omega$, a dual mesh is also introduced to realize a one-to-one pairing between edges and faces along with the transfer of orientation. For instance, outer-oriented dual faces are attached to inner-oriented primal edges, and so on. The primal and dual mesh do not play symmetric roles. The primal mesh is the one produced by the mesh generator and is the only mesh that needs to be seen by the end user. This mesh carries the information on the domain geometry, boundary conditions, and material properties. The dual mesh is used only in the intimate construction of the scheme, and, in general, there are several possibilities to build it.

The Section 2 introduces the classical ingredients of the discrete setting, namely the de Rham (or reduction) maps defining the degrees of freedom and the discrete differential operators along with their key structural properties (commuting with de Rham maps, adjunction of gradient and divergence). Then, following Bossavit [7], number five, and Perot and Subramanian [31], we introduce two families of schemes depending on the positioning of the potential degrees of freedom. Choosing a positioning on primal vertices leads to vertex-based schemes, while choosing a positioning on dual vertices (which are in a one-to-one pairing with primal cells) leads to cell-based schemes (the terminology is chosen to emphasize the salient role of the primal mesh). Both the vertex- and cell-based schemes admit two realizations (yielding, in general, distinct discrete solutions), one involving a Symmetric Positive Definite (SPD) system and the other a saddle-point system. Each of these four systems involves a specific discrete Hodge operator. Except in some particular cases (orthogonal meshes and isotropic conductivity) where a diagonal discrete Hodge operator can be built in the spirit of Covolume (Hu and Nicolaides [27]) and, more recently, Discrete Exterior Calculus (Desbrun et al. [16]) schemes, the discrete Hodge operator is, in general, sparse and SPD. A natural way to build this operator is through a cellwise assembly of local operators. In what follows, we focus on the two cases where the assembly is performed on primal cells, namely the vertex-based scheme in SPD form and the cell-based scheme in saddle-point form.

Sections 3 and 4 are devoted to the analysis of the vertex-based scheme in SPD form. The Section 3 develops an algebraic viewpoint. Firstly, we recall the basic error estimate in terms of the consistency error related to the lack of commuting property between the discrete Hodge operator and the de Rham maps. Then, we state the two design conditions on the local discrete Hodge operators, namely, on each primal cell, a stability condition and a $\mathbb{P}_{0}$-consistency condition. Then, under some mesh regularity assumptions, we establish new discrete functional analysis results, namely the discrete counterpart of the well-known Sobolev embeddings. 
While only the discrete Poincaré inequality is used herein (providing stability for the discrete problem), the more general result is important in view of nonlinear problems. Finally, we complete the analysis by establishing a first-order error estimate in discrete energy and complementary energy norms for smooth solutions in Sobolev spaces. A similar error estimate has been derived recently by Codecasa and Trevisan [15] under the stronger, piecewise Lipschitz assumption on the exact gradient and flux. We close Section 3 by showing that the present vertex-based scheme fits into the general framework of nodal Mimetic Finite Difference (MFD) schemes analyzed by Brezzi et al. [8].

The Section 4 adopts a more specific viewpoint to design the local discrete Hodge operator on polyhedral meshes with planar primal faces using a dual barycentric mesh. The idea is to introduce local reconstruction functions to reconstruct a gradient on each primal cell. Examples include Whitney forms on tetrahedral meshes using edge finite element functions and the Discrete Geometric Approach of Codecasa et al. [13,14] using piecewise constant functions on a simplicial submesh. An important observation is that we allow for nonconforming local gradient reconstructions, while existing literature has mainly focused on conforming reconstructions (see, e.g., Arnold et al. [3], Back [4], Buffa and Christiansen [10], Christiansen [11], Gillette [25], and Kreeft et al. [29]). We first state the design conditions on the local reconstruction functions, following the ideas of Codecasa and Trevisan [15], and show that the local discrete Hodge operator then satisfies the algebraic design conditions of Section 3. This yields first-order error estimates on the reconstructed gradient and flux for smooth solutions. We also show that the present scheme fits into the theoretical framework of Approximate Gradient Schemes introduced by Eymard et al. [23]. Finally, we prove a second-order $L^{2}$-error estimate for the potential; to our knowledge, this is the first result of this type for vertex-based schemes on polyhedral meshes.

The Section 5 deals with the design and analysis of cell-based schemes, first under an algebraic viewpoint and then using local flux reconstruction on a dual barycentric mesh assuming planar primal faces. Our theoretical results are similar to those derived in Sections 3 and 4 for vertex-based schemes. The cell-based schemes fit the unified analysis framework derived by Droniou et al. [21], so that they constitute a specific instance of mixed Finite Volume (see Droniou and Eymard [20]) and cell-based Mimetic Finite Difference (see Brezzi et al. [9]) schemes. Finally, we present numerical results in Section 6 and draw some conclusions in Section 7. Appendices A and B contain the proof of some technical results.

\section{Discrete Setting}

\subsection{Meshes and degrees of freedom}

The starting point is a discretization of the geometric domain $\Omega \subset \mathbb{R}^{3}$ in the form of a primal mesh $\mathrm{M}=$ $\{\mathrm{V}, \mathrm{E}, \mathrm{F}, \mathrm{C}\}$, where $\mathrm{V}$ collects primal vertices, $\mathrm{E}$ primal edges, $\mathrm{F}$ primal (possibly nonplanar) faces, and $\mathrm{C}$ primal cells. The mesh $\mathrm{M}$ has the structure of a cellular complex; see, e.g., Christiansen [11]. A generic element $\mathfrak{m}$ of $\mathrm{M}$ is a closed subset of $\Omega$ which is homeomorphic by a bi-Lipschitz map to the closed unit ball of $\mathbb{R}^{k}$ for some $0 \leq k \leq 3$ ( $k=0$ for vertices, $k=1$ for edges, $k=2$ for faces, and $k=3$ for cells). Its boundary, denoted $\partial \mathfrak{m}$, is the image of the unit sphere of $\mathbb{R}^{k}$ by the chosen homeomorphism, and its interior is $\mathfrak{m} \backslash \partial \mathfrak{m}$. The main properties of the cellular complex are as follows: elements of $\mathrm{M}$ have disjoint interiors; the intersection of two elements of $\mathrm{M}$ is a union of elements of $\mathrm{M}$; the boundary of any element of $\mathrm{M}$ is a union of elements of $\mathrm{M}$. For $\mathrm{A}$ any of the sets $\mathrm{V}, \mathrm{E}, \mathrm{F}, \mathrm{C}$ and an element $\mathfrak{m}$ of $\mathrm{M}$, we define the subset $A_{\mathfrak{m}}:=\{a \in A ; a \subset \partial \mathfrak{m}\}$ if $\mathfrak{m}$ has dimension larger than that of the elements of $\mathrm{A}$ and $A_{\mathfrak{m}}:=\{a \in A ; \mathfrak{m} \subset \partial a\}$ otherwise. For instance, $\mathrm{E}_{c}:=\{e \in \mathrm{E} ; e \subset \partial c\}$ and $\mathrm{C}_{e}:=\{c \in \mathrm{C} ; e \subset \partial c\}$, and so on.

In addition to the primal mesh, we consider a dual mesh $\widetilde{M}=\{\widetilde{V}, \widetilde{E}, \widetilde{F}, \widetilde{C}\}$. The precise way the dual mesh is built is only relevant in Sections 4 and 5.2. We only require that there is a one-to-one correspondence between primal vertices and dual cells, primal edges and dual faces, primal faces and dual edges, and primal cells and dual vertices. This correspondence is denoted $\tilde{c}(v), \tilde{f}(e)$, and so on; see Figure 1 . In what follows, we assume for simplicity that the conductivity is piecewise constant on the primal mesh (our results extend to piecewise 

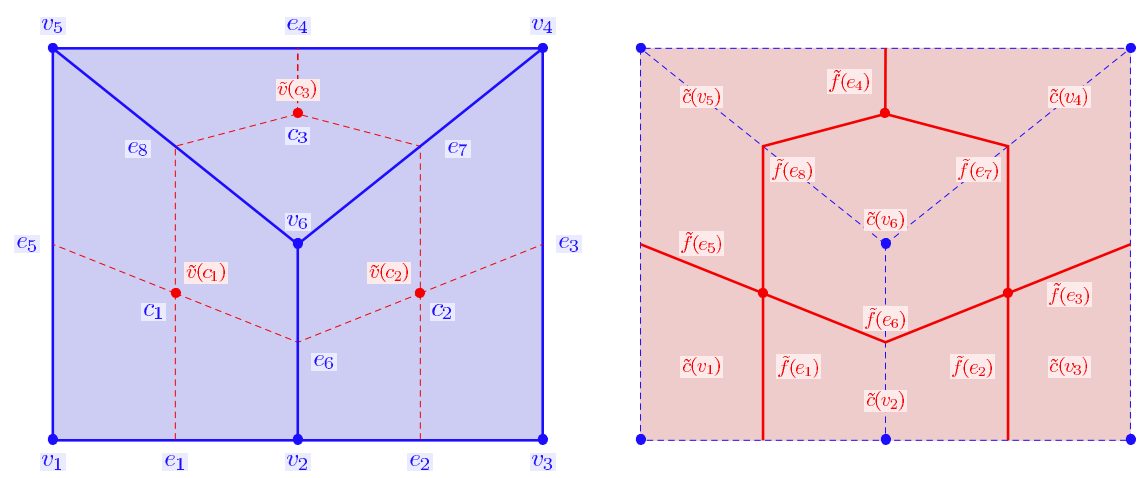

Figure 1. Example of primal (blue) and dual (red) meshes together with their corresponding geometric entities. Left: primal mesh in solid lines and dual mesh in dashed lines. Right: primal mesh in dashed lines and dual mesh in solid lines.

Lipschitz conductivity). We denote, respectively, $\lambda_{b, c}$ and $\lambda_{\sharp, c}$ the minimal and maximal eigenvalue of $\underline{\underline{\lambda}}$ on each primal cell $c \in \mathrm{C}$; the corresponding bounds on $\Omega$ are denoted $\lambda_{b}$ and $\lambda_{\sharp}$, respectively.

Recalling that the localization of the degrees of freedom reflects the physical nature of the fields, the degrees of freedom of the potential are attached to vertices, those of the gradient to edges, those of the flux to faces, and those of the source to cells. The first choice is whether the potential degrees of freedom are attached to primal or dual vertices. Once this choice is made, the localization of all the remaining degrees of freedom is fixed. Schemes in which the potential degrees of freedom are assigned to primal vertices are called vertex-based schemes. In such schemes, the degrees of freedom of the gradient are attached to primal edges, those of the flux to dual faces, and those of the source to dual cells. Primal edges are assigned a fixed inner orientation, and, for all $e \in \mathrm{E}$, the inner orientation of $e$ confers an outer orientation to its dual face $\tilde{f}(e)$. Alternatively, schemes in which the potential degrees of freedom are assigned to dual vertices (which are in one-to-one pairing with primal cells) are called cell-based schemes. In such schemes, the degrees of freedom of the gradient are attached to dual edges, those of the flux to primal faces, and those of the source to primal cells. Primal faces are assigned a fixed outer orientation, and, for all $f \in \mathrm{F}$, the outer orientation of $f$ confers an inner orientation to its dual edge $\tilde{e}(f)$ (the mechanism of orientation transfer emphasizes the salient role of the primal mesh).

\subsection{Vertex-based schemes}

In such schemes, the degrees of freedom of the potential, gradient, flux, and source live in finite-dimensional spaces denoted $\{\mathcal{V}, \mathcal{E}, \widetilde{\mathcal{F}}, \widetilde{\mathcal{C}}\}$, respectively. The notation for these spaces recalls the corresponding geometric entity, and their elements are often called cochains. The spaces $\mathcal{V}$ and $\widetilde{\mathcal{C}}$ have dimension \#V (the number of primal vertices), while the spaces $\mathcal{E}$ and $\widetilde{\mathcal{F}}$ have dimension \#E (the number of primal edges). Degrees of freedom are assigned through de Rham maps $\mathrm{R}_{\mathcal{A}}: S_{\mathcal{A}}(\Omega) \rightarrow \mathcal{A}$ with $\mathcal{A} \in\{\mathcal{V}, \mathcal{E}, \widetilde{\mathcal{F}}, \widetilde{\mathcal{C}}\}$ with

$$
\begin{array}{lll}
\forall v \in \mathrm{V}, & \left(\mathrm{R}_{\mathcal{V}}(p)\right)_{v}:=p(v), & \left(\mathrm{R}_{\tilde{\mathcal{C}}}(s)\right)_{\tilde{c}(v)}:=\int_{\tilde{c}(v)} s, \\
\forall e \in \mathrm{E}, \quad\left(\mathrm{R}_{\mathcal{E}}(\underline{g})\right)_{e}:=\int_{e} \underline{g} \cdot \underline{\tau}_{e}, \quad\left(\mathrm{R}_{\tilde{\mathcal{F}}}(\underline{\phi})\right)_{\tilde{f}(e)}:=\int_{\tilde{f}(e)} \underline{\phi} \cdot \underline{\nu_{\tilde{f}}(e)},
\end{array}
$$

where $\underline{\tau}_{e}$ is the unit tangent vector to $e$ fixing its inner orientation and $\underline{\nu}_{\tilde{f}(e)}$ is the unit normal vector to $\tilde{f}(e)$ whose orientation is given by $\underline{\tau}_{e}$. The domain $S_{\mathcal{A}}(\Omega)$ of the de Rham map $\mathrm{R}_{\mathcal{A}}$ can be specified using (broken) Sobolev spaces. To avoid technicalities, we leave them undefined and simple assume that the de Rham maps act on smooth enough functions; see, e.g., Zaglmayr [36]. 
In vertex-based schemes, the homogeneous Dirichlet boundary condition can be strongly enforced by discarding the potential degrees of freedom attached to boundary vertices and the gradient degrees of freedom attached to edges included in the boundary; the corresponding degrees of freedom attached to dual faces and dual cells are also discarded. For simplicity, we keep the same notation $\mathcal{V}, \mathcal{E}, \widetilde{\mathcal{F}}$, and $\widetilde{\mathcal{C}}$ in what follows. The relevant discrete differential operators are

$$
\text { GRAD }: \mathcal{V} \rightarrow \mathcal{E}, \quad \widetilde{\text { DIV }}: \widetilde{\mathcal{F}} \rightarrow \widetilde{\mathcal{C}},
$$

acting as follows:

$$
(\operatorname{GRAD}(\mathbf{p}))_{e}=\sum_{v \in \mathrm{V}_{e}} \iota_{v, e} \mathbf{p}_{v}, \quad(\widetilde{\operatorname{DIV}}(\phi))_{\tilde{c}}=\sum_{\tilde{f} \in \tilde{\mathrm{F}}_{\tilde{c}}} \iota_{\tilde{f}, \tilde{c}} \phi_{\tilde{f}}
$$

where $\iota_{v, e}=1$ if $\underline{\tau}_{e}$ points towards $v$ and $\iota_{v, e}=-1$ otherwise, $\iota_{\tilde{f}, \tilde{c}}=1$ if $\underline{\nu}_{\tilde{f}}$ points outwards $\tilde{c}$ and $\iota_{\tilde{f}, \tilde{c}}=-1$ otherwise, $\mathrm{V}_{e}=\{v \in \mathrm{V} ; v \subset \partial e\}$, and $\widetilde{\mathrm{F}}_{\tilde{c}}=\{\tilde{f} \in \widetilde{\mathrm{F}} ; \tilde{f} \subset \partial \tilde{c}\}$ (as per the above notation on sets of geometric entities). Owing to (2.4), the matrices representing the operators GRAD and $\widetilde{\text { IIV }}$ are incidence matrices (their entries are in $\{0, \pm 1\})$. Moreover, the discrete gradient and divergence operators GRAD and $\widetilde{\text { DIV }}$ satisfy the following classical properties, which are essential tools in the analysis of Compatible Discrete Operator schemes.

Lemma 2.1 (Commuting with de Rham map). There holds

$$
\operatorname{GRAD}\left(\mathrm{R}_{\mathcal{V}}\right)=\mathrm{R}_{\mathcal{E}}(\underline{\operatorname{grad}}), \quad \widetilde{\mathrm{DIV}}\left(\mathrm{R}_{\tilde{\mathcal{F}}}\right)=\mathrm{R}_{\widetilde{\mathcal{C}}}(\operatorname{div}) .
$$

Lemma 2.2 (Adjunction). Defining the discrete duality products

$$
\begin{array}{ll}
\forall \mathbf{g} \in \mathcal{E}, \forall \phi \in \widetilde{\mathcal{F}}, & \llbracket \mathbf{g}, \phi \rrbracket_{\mathcal{E} \tilde{\mathcal{F}}}:=\sum_{e \in \mathrm{E}} \mathbf{g}_{e} \phi_{\tilde{f}(e)}, \\
\forall \mathbf{p} \in \mathcal{V}, \forall \mathbf{s} \in \widetilde{\mathcal{C}}, \quad \llbracket \mathbf{p}, \mathbf{s} \rrbracket_{\mathcal{V} \tilde{\mathcal{C}}}:=\sum_{v \in \mathrm{V}} \mathbf{p}_{v} \mathbf{s}_{\tilde{\mathcal{c}}(v)},
\end{array}
$$

there holds

$$
\forall \mathbf{p} \in \mathcal{V}, \forall \phi \in \widetilde{\mathcal{F}}, \quad \llbracket \mathbf{p}, \widetilde{\operatorname{DIV}}(\phi) \rrbracket_{\mathcal{V} \tilde{\mathcal{C}}}=-\llbracket \operatorname{GRAD}(\mathbf{p}), \phi \rrbracket_{\mathcal{E} \tilde{\mathcal{F}}}
$$

Two approaches can be pursued for the discrete Hodge operator, either building $\mathrm{H}_{\lambda}^{\mathcal{E} \tilde{\mathcal{F}}}: \mathcal{E} \rightarrow \widetilde{\mathcal{F}}$, which leads to the SPD system: Find $\mathbf{p} \in \mathcal{V}$ such that

$$
-\widetilde{\mathrm{DIV}} \cdot \mathrm{H}_{\lambda}^{\varepsilon \tilde{\mathcal{F}}} \cdot \operatorname{GRAD}(\mathbf{p})=\mathrm{R}_{\widetilde{\mathcal{C}}}(s),
$$

or building $\mathrm{H}_{\lambda^{-1}}^{\tilde{\mathcal{F}}}: \widetilde{\mathcal{F}} \rightarrow \mathcal{E}$, which leads to the saddle-point problem: Find $(\mathbf{p}, \phi) \in \mathcal{V} \times \widetilde{\mathcal{F}}$ such that

$$
\left\{\begin{array}{l}
\mathrm{H}_{\lambda^{-} \mathcal{E}}^{\tilde{\mathcal{E}}}(\boldsymbol{\phi})+\mathrm{GRAD}(\mathbf{p})=0, \\
\widehat{\operatorname{DIV}}(\phi)=\mathrm{R}_{\widetilde{\mathcal{C}}}(s)
\end{array}\right.
$$

Since in general $\mathrm{H}_{\lambda}^{\mathcal{E}} \neq\left(\mathrm{H}_{\lambda^{-1}}^{\tilde{\mathcal{F}}}\right)^{-1}$, problems (2.9) and (2.10) lead to different discrete solutions (see, e.g., Gillette [25]). We also observe that (2.9) is a finite volume scheme on the dual mesh (also called vertex-centered finite volume scheme), since there is one unknown per dual cell and the mass balance is expressed on dual cells. Similarly, (2.10) is a mixed finite volume scheme on the dual mesh. For simplicity, we neglect quadrature errors on the source term $s$ and take $\mathrm{R}_{\widetilde{\mathcal{C}}}(s)$ on the right-hand side of (2.9) and (2.10). 


\subsection{Cell-based schemes}

In such schemes, the degrees of freedom of the potential, gradient, flux, and source live in finite-dimensional spaces denoted $\{\widetilde{\mathcal{V}}, \widetilde{\mathcal{E}}, \mathcal{F}, \mathcal{C}\}$, respectively. The spaces $\widetilde{\mathcal{V}}$ and $\mathcal{C}$ have dimension $\# \mathrm{C}$ (the number of primal cells), while the spaces $\widetilde{\mathcal{E}}$ and $\mathcal{F}$ have dimension \#F (the number of primal faces). Degrees of freedom are assigned through de Rham maps $\mathrm{R}_{\mathcal{A}}: S_{\mathcal{A}}(\Omega) \rightarrow \mathcal{A}$ with $\mathcal{A} \in\{\widetilde{\mathcal{V}}, \widetilde{\mathcal{E}}, \mathcal{F}, \mathcal{C}\}$ acting similarly to $(2.1)-(2.2)$. The relevant discrete differential operators are

$$
\widetilde{\mathrm{GRAD}}: \widetilde{\mathcal{V}} \rightarrow \widetilde{\mathcal{E}}, \quad \text { DIV }: \mathcal{F} \rightarrow \mathcal{C},
$$

acting similarly to (2.4). Enforcing the homogeneous Dirichlet boundary condition does not entail discarding degrees of freedom, but the discrete gradient operator explicitly accounts for the boundary condition on dual edges touching the boundary. Moreover, the discrete gradient and divergence operators $\widehat{\text { GRAD }}$ and DIV satisfy commuting and adjunction properties similar to those stated in Lemmata 2.1 and 2.2.

As for vertex-based schemes, two approaches can be pursued for the discrete Hodge operator, either building $\mathrm{H}_{\lambda}^{\tilde{\mathcal{F}} \mathcal{F}}: \widetilde{\mathcal{E}} \rightarrow \mathcal{F}$, which leads to the SPD system: Find $\mathbf{p} \in \widetilde{\mathcal{V}}$ such that

$$
-\mathrm{DIV} \cdot \mathrm{H}_{\lambda}^{\tilde{\tilde{F}}} \cdot \widetilde{\operatorname{GRAD}}(\mathbf{p})=\mathrm{R}_{\mathcal{C}}(s),
$$

or building $\mathrm{H}_{\lambda^{\mathcal{E}}-1}^{\mathcal{E}}: \mathcal{F} \rightarrow \widetilde{\mathcal{E}}$, which leads to the saddle-point problem: Find $(\mathbf{p}, \phi) \in \widetilde{\mathcal{V}} \times \mathcal{F}$ such that

$$
\left\{\begin{array}{l}
\mathrm{H}_{\lambda}^{\mathcal{F} \tilde{\mathcal{E}}}(\boldsymbol{\phi})+\widetilde{\operatorname{GRAD}}(\mathbf{p})=0, \\
\operatorname{DIV}(\phi)=\mathrm{R}_{\mathcal{C}}(s) .
\end{array}\right.
$$

Since in general $\mathrm{H}_{\lambda}^{\tilde{\mathcal{F}} \mathcal{F}} \neq\left(\mathrm{H}_{\lambda}^{\mathcal{F}} \tilde{\mathcal{E}}^{-1}\right)^{-1}$, problems (2.12) and (2.13) lead to different discrete solutions. We also observe that (2.12) is a finite volume scheme on the primal mesh (also called cell-centered finite volume scheme), since there is one unknown per primal cell and the mass balance is expressed on primal cells. Similarly, (2.13) is a mixed finite volume scheme on the primal mesh. As above, we neglect quadrature errors on the source term $s$ and take $\mathrm{R}_{\mathcal{C}}(s)$ on the right-hand side of (2.12) and (2.13).

\subsection{Designing the discrete Hodge operator}

In both vertex- and cell-based schemes, the key point is the design of the discrete Hodge operator, each choice leading to a specific scheme. In some particular situations, such as orthogonal meshes and isotropic conductivity, a diagonal discrete Hodge operator can be built for all schemes (2.9) to (2.13). For instance, for the vertex-based scheme (2.9), a natural choice is

$$
\left(\mathrm{H}_{\lambda}^{\tilde{\mathcal{F}}}\right)_{\tilde{f}(e), e}=\frac{1}{|e|} \sum_{c \in \mathrm{C}_{e}}|\tilde{f}(e) \cap c| \lambda_{c}, \quad \forall e \in \mathrm{E},
$$

(with an arithmetic average of the conductivity), while for the cell-based scheme (2.13), a natural choice is

$$
\left(\mathrm{H}_{\lambda^{-1}}^{\mathcal{F} \tilde{\tilde{\varepsilon}}}\right)_{\tilde{e}(f), f}=\frac{1}{|f|} \sum_{c \in \mathrm{C}_{f}} \frac{|\tilde{e}(f) \cap c|}{\lambda_{c}}, \quad \forall f \in \mathrm{F},
$$

(with an harmonic average of the conductivity). Moreover, a simple choice is $\mathrm{H}_{\lambda^{-1}}^{\tilde{\mathcal{E}}}=\left(\mathbf{H}_{\lambda}^{\mathcal{E} \tilde{\mathcal{F}}}\right)^{-1}$ for the vertexbased scheme (2.10) and $\mathbf{H}_{\lambda}^{\tilde{\mathcal{F}}}=\left(\mathbf{H}_{\lambda^{-1}}^{\mathcal{F}}\right)^{-1}$ for the cell-based scheme (2.12).

In general situations (non-orthogonal meshes or anisotropic conductivity), it is possible to design discrete Hodge operators that are sparse and SPD. A general way to proceed is to assemble local discrete Hodge operators defined at the cell level. It turns out that the assembly of the operators $\mathbf{H}_{\lambda}^{\mathcal{E}}$ and $\mathbf{H}_{\lambda}^{\mathcal{F} \tilde{\mathcal{E}}}$ is to be performed on primal cells, while that of $\mathrm{H}_{\lambda^{-1} \mathcal{E}}^{\tilde{\mathcal{F}}}$ and $\mathrm{H}_{\lambda}^{\tilde{\mathcal{E}} \mathcal{F}}$ on dual cells. In what follows, we focus on assembly on primal cells (on which the conductivity is constant, or, more generally, smooth), that is, on the vertex-based scheme (2.9) and the cell-based scheme (2.13). We now devise design conditions on the local discrete Hodge operators to analyze the two resulting schemes. 


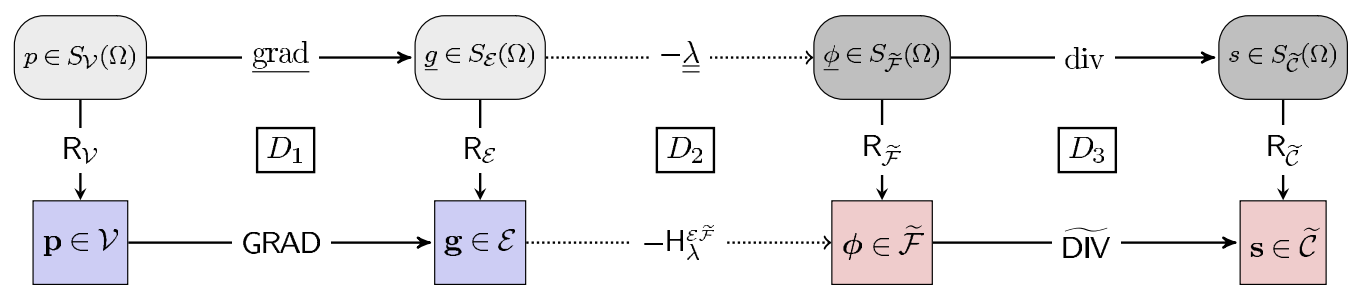

Figure 2. Tonti diagrams for the exact problem and the vertex-based scheme (2.9).

\section{AnAlysis of VerteX-BASED SCHEMES: ALGEBRAIC VIEWPoint}

In this section, we focus on the analysis of the vertex-based scheme (2.9). Recall that the relevant discrete differential operators are GRAD $: \mathcal{V} \rightarrow \mathcal{E}$ and $\widetilde{\mathrm{DIV}}: \widetilde{\mathcal{F}} \rightarrow \widetilde{\mathcal{C}}$, while the relevant discrete Hodge operator is $\mathrm{H}_{\lambda}^{\varepsilon \tilde{\mathcal{F}}}: \mathcal{E} \rightarrow \widetilde{\mathcal{F}}$. Since the operator $\mathrm{H}_{\lambda}^{\mathcal{E} \tilde{\mathcal{F}}}$ is, by construction, SPD, it is meaningful to consider the following discrete norms: For all $\mathbf{g} \in \mathcal{E}$ and all $\phi \in \widetilde{\mathcal{F}}$, recalling the definition (2.6) of the duality product $\llbracket \cdot, \cdot \rrbracket_{\mathcal{E} \tilde{\mathcal{F}}}$,

$$
\|\mathbf{g}\|_{\lambda}^{2}:=\llbracket \mathbf{g}, \mathrm{H}_{\lambda}^{\mathcal{E} \tilde{\mathcal{F}}}(\mathrm{g}) \rrbracket_{\mathcal{E} \tilde{\mathcal{F}}}, \quad\|\phi\|_{\lambda^{-1}}^{2}:=\llbracket\left(\mathrm{H}_{\lambda}^{\mathcal{E}}\right)^{-1}(\phi), \phi \rrbracket_{\mathcal{E} \tilde{\mathcal{F}}} .
$$

Owing to the Cauchy-Schwarz inequality, we infer

$$
\llbracket \mathrm{g}, \phi \rrbracket_{\mathcal{E} \tilde{\mathcal{F}}} \leq\|\mathrm{g}\|\left\|_{\lambda}\right\| \phi \|_{\lambda^{-1}} .
$$

The norms defined in (3.1) are discrete counterparts of the so-called energy and complementary energy norms, defined, for all $\underline{g}, \underline{\phi} \in\left[L^{2}(\Omega)\right]^{3}$, by $\|\underline{g}\|_{\lambda}^{2}:=\int_{\Omega} \underline{g} \cdot \underline{\underline{\lambda}} \underline{g}$ and $\|\underline{\phi}\|_{\lambda^{-1}}^{2}:=\int_{\Omega} \underline{\underline{\lambda}}^{-1} \underline{\phi} \cdot \underline{\phi}$.

\subsection{Consistency error}

The Tonti diagrams for the exact problem and the discrete scheme are linked through the de Rham maps as shown in Figure 2. Owing to Lemma 2.1, the diagrams $D_{1}$ and $D_{3}$ are commutative, but this is (in general) not the case for $D_{2}$. This lack of commutation produces a consistency error. This fact has been recognized in the seminal work of Bossavit [7], no. 3, and Hiptmair [26]; see also Codecasa and Trevisan [15].

Lemma 3.1 (Basic error bound). Let $g$ be the exact gradient and $\phi$ be the exact flux. Let $\mathbf{g}$ be the discrete gradient and $\phi$ the discrete flux resulting from (2.9). Then, provided $\overline{\mathrm{R}}_{\mathcal{E}}(\underline{g})$ and $\mathrm{R}_{\tilde{\mathcal{F}}}(\underline{\phi})$ are well-defined,

$$
\max \left(\left\|\mathrm{R}_{\mathcal{E}}(\underline{g})-\mathbf{g}\right\|_{\lambda},\left\|\mathrm{R}_{\tilde{\mathcal{F}}}(\underline{\phi})-\phi\right\|_{\lambda^{-1}}\right) \leq\|\lfloor\lambda, \mathrm{R}\rceil(\underline{g})\|_{\lambda^{-1}},
$$

where $\lfloor\lambda, \mathrm{R}\rceil:=\mathrm{H}_{\lambda}^{\varepsilon \tilde{\mathcal{F}}} \mathrm{R}_{\mathcal{E}}-\mathrm{R}_{\tilde{\mathcal{F}}} \underline{\lambda}$ is the commuting operator for diagram $D_{2}$ of Figure 2.

Proof. For completeness, we sketch the proof. Owing to the commuting properties of Lemma 2.1,

$$
\begin{aligned}
\mathrm{R}_{\mathcal{E}}(\underline{g})-\mathbf{g} & =\operatorname{GRAD}\left(\mathrm{R}_{\mathcal{V}}(p)\right)-\operatorname{GRAD}(\mathbf{p})=\operatorname{GRAD}\left(\mathrm{R}_{\mathcal{V}}(p)-\mathbf{p}\right), \\
\widetilde{\operatorname{DIV}}\left(\mathrm{R}_{\widetilde{\mathcal{F}}}(\underline{\phi})-\phi\right) & =\mathrm{R}_{\widetilde{\mathcal{C}}}(\operatorname{div} \underline{\phi})-\mathrm{R}_{\widetilde{\mathcal{C}}}(s)=0,
\end{aligned}
$$

where $p$ and $\mathbf{p}$, respectively, stand for the exact and discrete potentials. The adjunction of gradient and divergence of Lemma 2.2 then yields

$$
\llbracket \mathrm{R}_{\mathcal{E}}(\underline{g})-\mathbf{g}, \mathrm{R}_{\tilde{\mathcal{F}}}(\underline{\phi})-\phi \rrbracket_{\mathcal{E} \tilde{\mathcal{F}}}=0 .
$$

Moreover, a direct calculation shows that

$$
\mathrm{R}_{\tilde{\mathcal{F}}}(\underline{\phi})-\phi=-\mathrm{R}_{\tilde{\mathcal{F}}}(\underline{\lambda} \underline{g})+\mathrm{H}_{\lambda}^{\mathcal{E} \tilde{\mathcal{F}}}(\mathrm{g})=\lfloor\lambda, \mathrm{R}\rceil(\underline{g})-\mathrm{H}_{\lambda}^{\mathcal{E} \tilde{\mathcal{F}}}\left(\mathrm{R}_{\mathcal{E}}(\underline{g})-\mathrm{g}\right) .
$$

Applying the discrete duality product $\left[\mathrm{R}_{\mathcal{E}}(\underline{g})-\mathbf{g}, \cdot \rrbracket_{\mathcal{E} \tilde{\mathcal{F}}}\right.$ to $(3.5)$, using the definition of the discrete norm $\|\cdot\|_{\lambda}$ and (3.4), we obtain $\left\|\mathrm{R}_{\mathcal{E}}(\underline{g})-\mathbf{g}\right\|_{\lambda}^{2}=\llbracket \mathrm{R}_{\mathcal{E}}(\underline{g})-\mathbf{g},\lfloor\lambda, \mathrm{R}\rceil(\underline{g}) \rrbracket_{\mathcal{E} \tilde{\mathcal{F}}}$. Using the Cauchy-Schwarz inequality (3.2) yields the bound on the discrete energy error. The other bound is obtained similarly. 


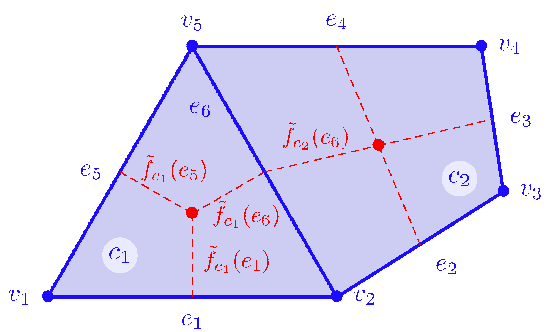

Figure 3. Geometric entities for local discrete Hodge operator.

\subsection{Local discrete Hodge operators and their properties}

As discussed in Section 2.4, the discrete Hodge operator $\mathrm{H}_{\lambda}^{\mathcal{E} \tilde{\mathcal{F}}}$ can be built by a cellwise assembly of local discrete Hodge operators attached to primal cells. Let $c \in \mathrm{C}$. Letting $\mathrm{E}_{c}:=\{e \in \mathrm{E} ; e \subset \partial c\}$, we define, for all $e \in \mathrm{E}_{c}$, the dual subface

$$
\tilde{f}_{c}(e):=\tilde{f}(e) \cap c,
$$

and consider the set $\widetilde{\mathrm{F}}_{c}:=\left\{\tilde{f}_{c}(e) ; e \in \mathrm{E}_{c}\right\}$; see Figure 3. The local de Rham maps $\mathrm{R}_{\mathcal{E}_{c}}: S_{\mathcal{E}}(c) \rightarrow \mathcal{E}_{c}$ and $\mathrm{R}_{\widetilde{\mathcal{F}}_{c}}: S_{\widetilde{\mathcal{F}}}(c) \rightarrow \widetilde{\mathcal{F}}_{c}$, defined for smooth enough functions in $c$, are such that, for all $e \in \mathrm{E}_{c}$,

$$
\left(\mathrm{R}_{\mathcal{E}_{c}}(\underline{g})\right)_{e}:=\int_{e} \underline{g} \cdot \underline{\tau}_{e}, \quad\left(\mathrm{R}_{\tilde{\mathcal{F}}_{c}}(\underline{\phi})\right)_{\tilde{f}_{c}(e)}:=\int_{\tilde{f}_{c}(e)} \underline{\phi} \cdot \underline{\nu}_{\tilde{f}_{c}(e)},
$$

where the unit normal $\underline{\nu}_{\tilde{f}_{c}(e)}$ is consistently oriented by $\underline{\tau}_{e}$. Local degrees of freedom in $c$ for the gradient and the flux belong to the spaces $\mathcal{E}_{c}$ and $\widetilde{\mathcal{F}}_{c}$, respectively, both of dimension $\# \mathrm{E}_{c}$. We introduce the local duality product

$$
\forall \mathbf{g} \in \mathcal{E}_{c}, \forall \phi \in \widetilde{\mathcal{F}}_{c}, \quad \llbracket \mathrm{g}, \phi \rrbracket_{\mathcal{E}_{c} \tilde{\mathcal{F}}_{c}}:=\sum_{e \in \mathrm{E}_{c}} \mathrm{~g}_{e} \phi_{\tilde{f}_{c}(e)} .
$$

The assembly of the operator $\mathrm{H}_{\lambda}^{\mathcal{E}}$ takes the form

$$
\mathrm{H}_{\lambda}^{\mathcal{E} \tilde{\mathcal{F}}}=\sum_{c \in \mathrm{C}} \mathrm{P}_{\mathcal{E}, c}^{*} \cdot \mathrm{H}_{\lambda}^{\mathcal{E}_{c} \tilde{\mathcal{F}}_{c}} \cdot \mathrm{P}_{\mathcal{E}, c}
$$

with local discrete Hodge operators $\mathrm{H}_{\lambda}^{\mathcal{E}_{c} \tilde{\mathcal{F}}_{c}}: \mathcal{E}_{c} \rightarrow \widetilde{\mathcal{F}}_{c}$, while $\mathrm{P}_{\mathcal{E}, c}: \mathcal{E} \ni \mathbf{g} \mapsto \mathbf{g}_{c}:=\mathrm{P}_{\mathcal{E}, c}(\mathbf{g}) \in \mathcal{E}_{c}$ is the (full-rank) map from global to local degrees of freedom on edges and $\mathrm{P}_{\mathcal{E}, c}^{*}$ its adjoint. We observe that the summation in (3.9) accounts for the fact that dual faces are shared by various primal cells. The two properties to be satisfied by the local operators $\mathrm{H}_{\lambda}^{\mathcal{E}_{c} \tilde{\mathcal{F}}_{c}}$ are the following: For all $c \in \mathrm{C}$,

(A1v) [Stability]. $\mathrm{H}_{\lambda}^{\mathcal{E}_{c} \tilde{\mathcal{F}}_{c}}$ is symmetric positive definite, and there is $\eta>0$ such that, for all $\mathbf{g} \in \mathcal{E}_{c}$,

$$
\eta \lambda_{b, c}\|\mathbf{g}\|_{2, \mathcal{E}_{c}}^{2} \leq \llbracket \mathbf{g}, \mathrm{H}_{\lambda}^{\varepsilon_{c} \tilde{\mathcal{F}}_{c}}(\mathbf{g}) \rrbracket_{\mathcal{E}_{c} \tilde{\mathcal{F}}_{c}} \leq \eta^{-1} \lambda_{\sharp, c}\|\mathbf{g}\|_{2, \mathcal{E}_{c}}^{2}, \quad\|\mathbf{g}\|_{2, \mathcal{E}_{c}}^{2}:=\sum_{e \in \mathrm{E}_{c}}\left|\tilde{f}_{c}(e) \| e\right|\left(\frac{\mathbf{g}_{e}}{|e|}\right)^{2} .
$$

(A2v) $\left[\mathbb{P}_{0}\right.$-consistency]. Introducing the local commuting operator $\lfloor\lambda, \mathrm{R}]_{c}:=\mathrm{H}_{\lambda}^{\mathcal{E}_{c} \tilde{\mathcal{F}}_{c}} \mathrm{R}_{\mathcal{E}_{c}}-\mathrm{R}_{\widetilde{\mathcal{F}}_{c}} \underline{\underline{\lambda}}$, there holds, for all $\underline{G}$ constant in $c,\lfloor\lambda, \mathrm{R}\rceil_{c}(\underline{G})=0$.

Since (3.9) yields $\llbracket \mathbf{g}, \mathrm{H}_{\lambda}^{\mathcal{E}}(\mathbf{g}) \rrbracket_{\mathcal{E} \tilde{\mathcal{F}}}=\sum_{c \in \mathrm{C}} \llbracket \mathrm{P}_{\mathcal{E}, c}(\mathbf{g}), \mathrm{H}_{\lambda}^{\mathcal{E}_{c} \tilde{\mathcal{F}}_{c}} \mathrm{P}_{\mathcal{E}, c}(\mathbf{g}) \rrbracket_{\mathcal{E}_{c} \tilde{\mathcal{F}}_{c}}$ for all $\mathbf{g} \in \mathcal{E}$, summing (A1v) over mesh cells and recalling the definition (3.1) of the discrete energy norm, we infer

$$
\eta \lambda_{b}\|\mathbf{g}\|_{2, \mathcal{E}}^{2} \leq\|\mathbf{g}\|_{\lambda}^{2} \leq \eta^{-1} \lambda_{\sharp}\|\mathbf{g}\|_{2, \mathcal{E}}^{2}, \quad\|\mathbf{g}\|_{2, \mathcal{E}}^{2}:=\sum_{c \in \mathrm{C}}\|\mathbf{g}\|_{2, \mathcal{E}_{c}}^{2} .
$$

Moreover, (A2v) transforms the result of Lemma 3.1 into the following tighter error bound. 
Lemma 3.2 (Tighter error bound). Let $\left[\mathbb{P}_{0}(\mathrm{C})\right]^{3}$ be spanned by piecewise constant functions on the primal mesh. Then, provided $\mathrm{R}_{\mathcal{E}}(\underline{g})$ and $\mathrm{R}_{\tilde{\mathcal{F}}}(\underline{\phi})$ are well-defined,

$$
\max \left(\left\|\mathrm{R}_{\mathcal{E}}(\underline{g})-\mathrm{g}\right\|\left\|_{\lambda},\right\| \mathrm{R}_{\tilde{\mathcal{F}}}(\underline{\phi})-\phi \|_{\lambda^{-1}}\right) \leq \inf _{\underline{G} \in\left[\mathbb{P}_{0}(\mathrm{C})\right]^{3}}\left\{\sum_{c \in \mathrm{C}}\left\|\lfloor\lambda, \mathrm{R}\rceil_{c}\left(\left.(\underline{g}-\underline{G})\right|_{c}\right)\right\|_{\lambda^{-1}, c}^{2}\right\}^{1 / 2}
$$

with the local norm $\left.\|\phi\|_{\lambda^{-1}, c}^{2}:=\llbracket\left(\mathrm{H}_{\lambda}^{\mathcal{E}_{c} \tilde{\mathcal{F}}_{c}}\right)^{-1}(\phi), \phi\right]_{\mathcal{E}_{c} \tilde{\mathcal{F}}_{c}}$ for all $\phi \in \widetilde{\mathcal{F}}_{c}$.

Proof. Owing to the algebraic result of Codecasa and Trevisan (see Theorem 9 in[15]) there holds, for all $\phi \in \widetilde{\mathcal{F}}$,

$$
\|\phi\|_{\lambda^{-1}}^{2}=\llbracket\left(\mathrm{H}_{\lambda}^{\mathcal{E} \tilde{\mathcal{F}}}\right)^{-1}(\phi), \phi \rrbracket_{\mathcal{E} \tilde{\mathcal{F}}} \leq \sum_{c \in \mathrm{C}} \llbracket\left(\mathrm{H}_{\lambda}^{\mathcal{E}_{c} \tilde{\mathcal{F}}_{c}}\right)^{-1}\left(\mathrm{P}_{\widetilde{\mathcal{F}}, c}(\phi)\right), \mathrm{P}_{\widetilde{\mathcal{F}}, c}(\phi) \rrbracket_{\mathcal{E}_{c} \tilde{\mathcal{F}}_{c}}=\sum_{c \in \mathrm{C}}\left\|\mathrm{P}_{\widetilde{\mathcal{F}}, c}(\phi)\right\|_{\lambda^{-1}, c}^{2}
$$

where $\mathrm{P}_{\widetilde{\mathcal{F}}, c}$ acts as $\mathrm{P}_{\mathcal{E}, c}$ mapping global to local degrees of freedom on dual faces. Applying this result to $\phi=\lfloor\lambda, \mathbf{R}\rceil \underline{g})$ and since $\mathrm{P}_{\widetilde{\mathcal{F}}, c}(\lfloor\lambda, \mathrm{R}\rceil(\underline{g}))=\lfloor\lambda, \mathrm{R}\rceil_{c}\left(\left.g\right|_{c}\right)$, we infer

$$
\|\lfloor\lambda, \mathrm{R}\rceil(\underline{g})\|_{\lambda^{-1}}^{2} \leq \sum_{c \in \mathrm{C}}\left\|\lfloor\lambda, \mathrm{R}\rceil_{c}\left(\left.g\right|_{c}\right)\right\|_{\lambda^{-1}, c}^{2} .
$$

We conclude using the result of Lemma 3.1 together with $(\mathbf{A 2 v})$.

Remark 3.3 (Exact solution for piecewise affine functions). Assuming that the exact solution turns out to be piecewise affine on the primal mesh, it is clear from (3.12) that the solution of the scheme is the image by the de Rham map of the exact solution. Indeed, since $\underline{g} \in\left[\mathbb{P}_{0}(\mathrm{C})\right]^{3}$, the infimum in (3.12) is zero so that $\mathrm{g}=\mathrm{R}_{\mathcal{E}}(\underline{g})$ and $\phi=\mathrm{R}_{\widetilde{\mathcal{F}}}(\underline{\phi})$; moreover, owing to the Poincaré inequality (3.19) below, $\mathbf{p}=\mathrm{R}_{\mathcal{V}}(p)$. This property can be used by practitioners to verify the scheme on coarse meshes, see, e.g., Eymard et al. [23], page 277.

\subsection{Discrete functional analysis and discrete stability of the scheme}

\subsubsection{Assumptions on mesh regularity}

When working with polyhedral meshes, a rather general way to formulate a sufficient condition on mesh regularity is the following:

(M1) There exists a matching simplicial submesh that is shape-regular in the usual finite element sense and that is a common refinement of both primal and dual meshes with each primal and dual cell containing a uniformly bounded number of subsimplices.

The simplicial submesh in Assumption (M1), which appears naturally if a dual barycentric mesh is used (see Section 4.1), is convenient in the convergence analysis to exploit classical techniques of finite element approximation theory. Note that within Assumption (M1), primal faces which are nonplanar are necessarily composed of triangles. For the discrete functional analysis results derived in Section 3.3.2, Assumption (M1) can be avoided, the only mesh regularity requirement being the existence of a positive real number $\gamma$ such that, for all $v \in \mathrm{V}$,

$$
\gamma \sum_{e \in \mathrm{E}_{v}}|e||\tilde{f}(e)| \leq|\tilde{c}(v)|
$$

Furthermore, Assumption (M1) can also be avoided in the convergence analysis under somewhat stronger regularity assumptions on the exact solution, see Remark 3.10. 


\subsubsection{Discrete functional analysis}

The discrete functional analysis results presented in this section follow the lines of Eymard et al. [22] for finite volume schemes (see also Di Pietro and Ern [17] for discontinuous Galerkin schemes). To allow for more generality, we consider in this section an arbitrary dimension $d>1$. For a real number $1 \leq p<+\infty$, we define the following discrete norms: for all $\mathbf{p} \in \mathcal{V}$ and all $\mathbf{g} \in \mathcal{E}$,

$$
\|\mathbf{p}\|_{p, \mathcal{V}}^{p}:=\sum_{v \in \mathrm{V}}\left|\tilde { c } ( v ) \| \mathbf { p } _ { v } | ^ { p } , \quad \| \mathbf { g } \left\|_{p, \mathcal{E}}^{p}:=\sum_{c \in \mathrm{C}} \sum_{e \in \mathrm{E}_{c}}\left|\tilde{f}_{c}(e) \| e\right|\left(\frac{\left|\mathbf{g}_{e}\right|}{|e|}\right)^{p} .\right.\right.
$$

Assuming there is a smooth field $p$ such that $\mathbf{p}=\mathrm{R}_{\mathcal{V}}(p)$ and $\mathbf{g}=\operatorname{GRAD}(\mathbf{p})$, the above norms are discrete counterparts of the continuous norms $\|p\|_{L^{p}(\Omega)}$ and $\|\underline{\operatorname{grad}} p\|_{L^{p}(\Omega)^{d}}$. Owing to the Hölder inequality and since (3.13) yields $\sum_{e \in \mathrm{E}}|\tilde{f}(e)||e| \leq(2 \gamma)^{-1}|\Omega|$, we infer, for all real numbers $1 \leq q \leq r$,

$$
\|\mathbf{p}\|_{q, \mathcal{V}} \leq|\Omega|^{\frac{r-q}{r q}}\|\mathbf{p}\|_{r, \mathcal{V}}, \quad\|\mathbf{g}\|_{q, \mathcal{E}} \leq\left((2 \gamma)^{-1}|\Omega|\right)^{\frac{r-q}{r q}}\|\mathbf{g}\|_{r, \mathcal{E}}
$$

For all $\mathbf{p} \in \mathcal{V}, \mathbf{L}_{\mathcal{V}}^{0}(\mathbf{p})$ is defined as the piecewise constant function on the dual mesh such that, for all $v \in \mathrm{V}$, $\left.\mathrm{L}_{\mathcal{V}}^{0}(\mathbf{p})\right|_{\tilde{c}(v)}:=\mathbf{p}_{v}$, and extended by zero on $\mathbb{R}^{d} \backslash \cup_{v \in \mathrm{V}} \tilde{c}(v)$. It is clear that $\mathrm{L}_{\mathcal{V}}^{0}(\mathbf{p}) \in L^{1}\left(\mathbb{R}^{d}\right)$. For a function $v \in L^{1}\left(\mathbb{R}^{d}\right)$, its $\|\cdot\|_{\mathrm{BV}}$-norm is defined as

$$
\|v\|_{\mathrm{BV}}:=\sup _{\underline{\psi} \in C_{0}^{\infty}\left(\mathbb{R}^{d}, \mathbb{R}^{d}\right),\|\underline{\psi}\|_{L^{\infty}\left(\mathbb{R}^{d}\right)} \leq 1} \int_{\mathbb{R}^{d}} v \operatorname{div}(\underline{\psi}) .
$$

Recall that the discrete operator GRAD explicitly accounts for the homogeneous Dirichlet boundary condition on primal edges touching the boundary.

Theorem 3.4 (Bound on $\|\cdot\|_{\mathrm{BV}}$-norm). For all $\mathbf{p} \in \mathcal{V}$, there holds

$$
\left\|L_{\mathcal{V}}^{0}(\mathbf{p})\right\|_{B V} \leq \sqrt{d}\|\operatorname{GRAD}(\mathbf{p})\|_{1, \mathcal{E}}
$$

Proof. Owing to the adjunction property of Lemma 2.2 and the commuting property of Lemma 2.1 on the DIV operator, we infer

$$
\int_{\mathbb{R}^{d}} \mathrm{~L}_{\mathcal{V}}^{0}(\mathbf{p}) \operatorname{div}(\underline{\psi})=\llbracket \mathbf{p}, \mathrm{R}_{\tilde{\mathcal{C}}}(\operatorname{div}(\underline{\psi})) \rrbracket_{\mathcal{V} \tilde{\mathcal{C}}}=\llbracket \mathbf{p}, \widetilde{\operatorname{DIV}}\left(\mathrm{R}_{\tilde{\mathcal{F}}}(\underline{\psi})\right) \rrbracket_{\mathcal{V} \tilde{\mathcal{C}}}=-\llbracket \operatorname{GRAD}(\mathbf{p}), \mathrm{R}_{\tilde{\mathcal{F}}}(\underline{\psi}) \rrbracket_{\mathcal{E} \tilde{\mathcal{F}}} .
$$

Since all the components of $\underline{\psi}$ are bounded by 1 , we infer, for all $e \in \mathrm{E},\left|\mathrm{R}_{\tilde{\mathcal{F}}}(\underline{\psi})_{\tilde{f}(e)}\right| \leq \sqrt{d}|\tilde{f}(e)|$, so that

$$
\left|\llbracket \operatorname{GRAD}(\mathbf{p}), \mathrm{R}_{\tilde{\mathcal{F}}}(\underline{\psi}) \rrbracket_{\mathcal{E} \tilde{\mathcal{F}}}\right| \leq \sqrt{d} \sum_{e \in \mathrm{E}}\left|(\operatorname{GRAD}(\mathbf{p}))_{e}\right||\tilde{f}(e)|=\sqrt{d} \sum_{e \in \mathrm{E}} \sum_{c \in \mathrm{C}_{e}}\left|(\operatorname{GRAD}(\mathbf{p}))_{e}\right|\left|\tilde{f}_{c}(e)\right|
$$

whence we infer (3.17) by inverting the order of the summations.

An important consequence of Theorem 3.4 is the following discrete Sobolev embedding. Herein, we only state the result, postponing the proof to Appendix A.

Theorem 3.5 (Discrete Sobolev embedding). Let $d>1$. Let $1 \leq p<+\infty$. Assume (3.13). Then, for all $q$ satisfying

- $1 \leq q \leq p^{*}:=\frac{p d}{d-p}$ if $1 \leq p<d$,

- $1 \leq q<\infty$ if $d \leq p<\infty$, 
there is $C_{p, q}$ such that, for all $\mathbf{p} \in \mathcal{V}$,

$$
\|\mathbf{p}\|_{q, \mathcal{V}} \leq C_{p, q}\|\operatorname{GRAD}(\mathbf{p})\|_{p, \mathcal{E}} .
$$

In particular, the choice $p=q=2$ leads to the discrete Poincaré inequality

$$
\|\mathbf{p}\|_{2, \mathcal{V}} \leq C_{2,2}\|\operatorname{GRAD}(\mathbf{p})\|_{2, \mathcal{E}} .
$$

A simple consequence of the discrete Poincaré inequality is the stability of the vertex-based scheme.

Corollary 3.6 (Discrete stability). Let $\mathbf{p} \in \mathcal{V}$ solve (2.9). There holds, with $C=\left(\eta \lambda_{b}\right)^{-1} C_{2,2}$,

$$
\|\operatorname{GRAD}(\mathbf{p})\|_{2, \mathcal{E}} \leq C\|s\|_{L^{2}(\Omega)} .
$$

Proof. Let $\mathbf{p} \in \mathcal{V}$ solve $(2.9)$. Setting $\phi=-\mathrm{H}_{\lambda}^{\varepsilon \tilde{\mathcal{F}}} \operatorname{GRAD}(\mathbf{p})$, there holds $\widetilde{\operatorname{DIV}}(\phi)=\mathrm{R}_{\widetilde{\mathcal{C}}}(s)$. Hence, using the adjunction property of Lemma 2.2 and the lower bound in (3.11),

$$
\llbracket \mathbf{p}, R_{\widetilde{\mathcal{C}}}(s) \rrbracket_{\mathcal{V} \tilde{\mathcal{C}}}=\llbracket \mathbf{p}, \widetilde{\operatorname{DIV}}(\phi) \rrbracket_{\mathcal{V} \tilde{\mathcal{C}}}=\llbracket \operatorname{GRAD}(\mathbf{p}), \mathrm{H}_{\lambda}^{\mathcal{E} \tilde{\mathcal{F}}} \operatorname{GRAD}(\mathbf{p}) \rrbracket_{\mathcal{\mathcal { F }} \tilde{\mathcal{F}}}=\|\operatorname{GRAD}(\mathbf{p})\|_{\lambda}^{2} \geq \eta \lambda_{b}\|\operatorname{GRAD}(\mathbf{p})\|_{2, \mathcal{E}}^{2} .
$$

Moreover, using two times the Cauchy-Schwarz inequality yields

$$
\llbracket \mathbf{p}, \mathrm{R}_{\tilde{\mathcal{C}}}(s) \rrbracket_{\mathcal{V} \tilde{\mathcal{C}}} \leq\|\mathbf{p}\|_{2, \mathcal{V}}\left(\sum_{v \in \mathrm{V}}|\tilde{c}(v)|^{-1}\left(\int_{\tilde{c}(v)} s\right)^{2}\right)^{1 / 2} \leq\|\mathbf{p}\|_{2, \mathcal{V}}\|s\|_{L^{2}(\Omega)} .
$$

We conclude using the discrete Poincaré inequality (3.19).

\subsection{Convergence rate for smooth solutions}

We now derive from the result of Lemma 3.2 a first-order convergence result for the discrete energy and complementary energy errors in the case of smooth solutions. In what follows, we often abbreviate $A \lesssim B$ the inequality $A \leq c B$ with positive $c$ whose value can change at each occurrence and is independent of any mesh size (but can depend on mesh regularity parameters and the stability parameter $\eta$ ).

Theorem 3.7 (Convergence rate for smooth solutions). Let $\underline{g}$ be the exact gradient and $\underline{\phi}$ the exact flux. Let $\mathrm{g}$ be the discrete gradient and $\boldsymbol{\phi}$ the discrete flux resulting from $(2.9)$. Let $H^{1}(\mathrm{C})$ be spanned by piecewise $H^{1}$ functions on the primal mesh. Assume $\underline{g}, \underline{\phi} \in\left[H^{1}(\mathrm{C})\right]^{3}$. Assume the meshes satisfy (M1). Let $h_{\mathrm{M}}$ denote the maximal diameter of primal cells. Then,

$$
\max \left(\left\|\mathrm{R}_{\mathcal{E}}(\underline{g})-\mathrm{g}\right\|\left\|_{\lambda},\right\| \mathrm{R}_{\widetilde{\mathcal{F}}}(\underline{\phi})-\phi \|_{\lambda^{-1}}\right) \lesssim h_{\mathrm{M}}\left(\lambda_{\sharp}^{1 / 2}\|\underline{g}\|_{H^{1}(\mathrm{C})^{3}}+\lambda_{\mathrm{b}}^{-1 / 2}\|\underline{\phi}\|_{H^{1}(\mathrm{C})^{3}}\right) .
$$

Proof. We preliminarily observe that $\mathrm{R}_{\mathcal{E}}(\underline{g})$ and $\mathrm{R}_{\tilde{\mathcal{F}}}(\underline{\phi})$ are well-defined since $\underline{g}, \underline{\phi} \in\left[H^{1}(\mathrm{C})\right]^{3}, \underline{g}$ is curl-free, and $\operatorname{div} \phi=s \in L^{2}(\Omega)$. We estimate the upper bound in (3.12) where we choose $\underline{G}$ as the $L^{2}$-orthogonal projection of the exact gradient $\underline{g}$ onto $\left[\mathbb{P}_{0}(\mathrm{C})\right]^{3}$. Since $\underline{\underline{\lambda}}$ is piecewise constant, $\underline{\Phi}:=-\underline{\underline{\lambda}} \underline{G}$ is the $L^{2}$-orthogonal projection of $\underline{\phi}$ onto $\left[\mathbb{P}_{0}(\mathrm{C})\right]^{3}$. Owing to the triangle inequality, we infer, for all $c \in \mathrm{C}$,

$$
\begin{aligned}
\|\|\lfloor, \mathrm{R}]_{c}\left(\left.(\underline{g}-\underline{G})\right|_{c}\right) \|_{\lambda^{-1}, c} \leq & \llbracket \mathrm{R}_{\mathcal{E}_{c}}\left(\left.(\underline{g}-\underline{G})\right|_{c}\right), \mathrm{H}_{\lambda}^{\mathcal{E}_{\varepsilon^{*}} \tilde{\mathcal{F}}_{c}}\left(\mathrm{R}_{\mathcal{E}_{c}}\left(\left.(\underline{g}-\underline{G})\right|_{c}\right)\right) \rrbracket_{\mathcal{E}_{c} \tilde{\mathcal{F}}_{c}} \\
& +\llbracket\left(\mathrm{H}_{\lambda}^{\mathcal{E}_{c} \tilde{\mathcal{F}}_{c}}\right)^{-1}\left(\mathrm{R}_{\widetilde{\mathcal{F}}_{c}}\left(\left.(\underline{\phi}-\underline{\Phi})\right|_{c}\right)\right), \mathrm{R}_{\widetilde{\mathcal{F}}_{c}}\left(\left.(\underline{\phi}-\underline{\Phi})\right|_{c}\right) \rrbracket_{\mathcal{E}_{c} \tilde{\mathcal{F}}_{c}} \\
\lesssim & \lambda_{\sharp, c} h_{c} \sum_{e \in \mathrm{E}_{c}}\left|T_{1, e}\right|^{2}+\lambda_{b, c}^{-1} h_{c}^{-1} \sum_{e \in \mathrm{E}_{c}}\left|T_{2, \tilde{f}_{c}(e)}\right|^{2},
\end{aligned}
$$


where we have used both bounds in (A1v) together with mesh regularity, and we have set

$$
T_{1, e}:=\left.\int_{e}(\underline{g}-\underline{G})\right|_{c} \cdot \underline{\tau}_{e}, \quad T_{2, \tilde{f}_{c}(e)}:=\left.\int_{\tilde{f}_{c}(e)}(\underline{\phi}-\underline{\Phi})\right|_{c} \cdot \underline{\nu}_{\tilde{f}_{c}(e)} .
$$

These two terms are estimated using classical finite element analysis tools applied on the simplicial submesh of each primal cell. Consider first $T_{1, e}$. Pick a (subsimplex) face $f$ of $c$ to which $e$ belongs. Since $g \in\left[H^{1}(c)\right]^{3}$, $g \in\left[L^{4}(f)\right]^{3}$. Using the result of Amrouche et al. [1], Lemma 4.7, let $\varphi_{e}$ result from the extension by zero to $\partial f$ of the characteristic function on $e$ followed by a lifting into $W^{\frac{1}{4}, \frac{4}{3}}(f)$. Observing that $\underline{g}-\underline{G}$ is curl-free in $c$, we infer, owing to mesh regularity,

$$
\left|T_{1, e}\right| \lesssim\left\|\left.(\underline{g}-\underline{G})\right|_{c} \times \underline{\nu}_{f}\right\|_{L^{4}(f)^{2}}\left\|\varphi_{e}\right\|_{W^{\frac{1}{4}, \frac{4}{3}(f)}} \lesssim h_{c}^{\frac{1}{2}}\left\|\left.(\underline{g}-\underline{G})\right|_{c}\right\|_{L^{4}(f)^{3}} \lesssim h_{c}^{\frac{1}{2}} \| \underline{g}_{H^{1}(c)^{3}} .
$$

The bound on $T_{2, \tilde{f}_{c}(e)}$ is simpler since mesh regularity yields

$$
\left|T_{2, \tilde{f}_{c}(e)}\right| \leq\left|\tilde{f}_{c}(e)\right|^{\frac{1}{2}}\|\underline{\phi}-\underline{\Phi}\|_{L^{2}\left(\tilde{f}_{c}(e)\right)^{3}} \lesssim\left|\tilde{f}_{c}(e)\right|^{\frac{1}{2}} h_{c}^{\frac{1}{2}}\|\underline{\phi}\|_{H^{1}(c)^{3}} \lesssim h_{c}^{\frac{3}{2}}\|\underline{\phi}\|_{H^{1}(c)^{3}} .
$$

The conclusion is straightforward.

Remark 3.8 (Estimate on the potential). Using the discrete Poincaré inequality (3.19), the lower bound in (3.11), and the commuting property of Lemma 2.1, we infer

$$
\left(C_{2,2}\right)^{-1}\left\|\mathrm{R}_{\mathcal{V}}(p)-\mathbf{p}\right\|_{2, \mathcal{V}} \leq\left\|\operatorname{GRAD}\left(\mathrm{R}_{\mathcal{V}}(p)-\mathbf{p}\right)\right\|_{2, \mathcal{E}} \leq\left(\eta \lambda_{b}\right)^{-1 / 2}\left\|\operatorname{GRAD}\left(\operatorname{R}_{\mathcal{V}}(p)-\mathbf{p}\right)\right\|_{\lambda}=\left(\eta \lambda_{b}\right)^{-1 / 2}\left\|\operatorname{R}_{\mathcal{E}}(\underline{g})-\mathbf{g}\right\|_{\lambda} .
$$

Hence, the right-hand side of (3.21) also bounds $\left(\eta \lambda_{b}\right)^{1 / 2}\left(C_{2,2}\right)^{-1}\left\|R_{\mathcal{V}}(p)-\mathbf{p}\right\|_{2, \mathcal{V}}$.

Remark 3.9 (Verifying the regularity assumption). The regularity assumption $g, \phi \in\left[H^{1}(\mathrm{C})\right]^{3}$ is satisfied if, for instance, the exact potential sits in $H^{2}(\Omega)$ and, more generally, if the potential is in $S_{\mathcal{V}}(\Omega)$ and is piecewise $H^{2}$ on a partition of $\Omega$ to which the primal mesh is conforming.

Remark 3.10 (Stronger regularity assumption). Simpler arguments can be deployed whenever $\underline{g}$ and $\underline{\phi}$ are bounded and Lipschitz, see Codecasa and Trevisan [15]. By exploiting this stronger regularity assumption, regularity can be formulated in terms of geometric requirements without resorting to the simplicial submesh. Indeed, the terms $T_{1, e}$ and $T_{2, \tilde{f}_{c}(e)}$ in the above proof can be readily estimated as $\left|T_{1, e}\right| \lesssim|e| h_{c} \operatorname{Lip}(\underline{g})$ and $\left|T_{2, \tilde{f}_{c}(e)}\right| \lesssim\left|\tilde{f}_{c}(e)\right| h_{c} \operatorname{Lip}(\underline{\phi})$, yielding $\left\|\mathrm{R}_{\mathcal{E}}(\underline{g}-\underline{G})\right\|_{\lambda} \lesssim \eta^{-1 / 2} \lambda_{\sharp}^{1 / 2} h_{\mathrm{M}}|\Omega|^{\frac{1}{2}} \operatorname{Lip}(\underline{g})$ (using $\left.|e|^{2} h_{c} \lesssim|c|\right)$ and $\| \mathrm{R}_{\tilde{\mathcal{F}}}(\underline{\phi}-$ $\underline{\Phi)} \|_{\lambda^{-1}} \lesssim \eta^{-1 / 2} \lambda_{b}^{-1 / 2} h_{\mathrm{M}} \operatorname{Lip}(\underline{\phi})$ (using $\left.h_{c}^{-1}\left|\tilde{f}_{c}(e)\right|^{2} \lesssim|c|\right)$.

\subsection{Link with nodal mimetic finite difference schemes}

We show in this section that the vertex-based scheme (2.9) belongs to the family of nodal MFD schemes analyzed recently by Brezzi et al. [8]. We first observe that, owing to the adjunction property (2.8), our scheme (2.9) can be cast into the following discrete variational form: find $\mathbf{p} \in \mathcal{V}$ such that

$$
\llbracket \operatorname{GRAD}(\mathbf{p}), \mathrm{H}_{\lambda}^{\mathcal{E} \tilde{\mathcal{F}}} \operatorname{GRAD}(\mathbf{q}) \rrbracket_{\mathcal{E} \tilde{\mathcal{F}}}=\llbracket \mathbf{q}, \mathrm{R}_{\widetilde{\mathcal{C}}}(s) \rrbracket_{\mathcal{V} \tilde{\mathcal{c}}}, \quad \forall \mathbf{q} \in \mathcal{V} .
$$

Let $c \in \mathrm{C}$ and let $\mathrm{V}_{c}:=\{v \in \mathrm{V} ; v \subset \partial c\}$. The corresponding space of degrees of freedom is denoted $\mathcal{V}_{c}$. Similarly to (2.4), we consider the local discrete gradient operator $\operatorname{GRAD}_{c}: \mathcal{V}_{c} \rightarrow \mathcal{E}_{c}$. Letting $\mathrm{P}_{\mathcal{V}, c}: \mathcal{V} \rightarrow \mathcal{V}_{c}$ be the (full-rank) map of global to local degrees of freedom on vertices, it is easily seen that the commuting property $\mathrm{GRAD}_{c} \mathrm{P}_{\mathcal{V}, c}=\mathrm{P}_{\mathcal{E}, c} \mathrm{GRAD}$ holds on $\mathcal{V}$ for all $c \in \mathrm{C}$. Then, for all $\mathbf{p}, \mathbf{q} \in \mathcal{V}_{c}$, we define the bilinear form

$$
\llbracket \mathbf{p}, \mathbf{q} \rrbracket_{c}^{\mathrm{MFD}}:=\left[\operatorname{GRAD}_{c}(\mathbf{p}), \mathrm{H}_{\lambda}^{\mathcal{E}_{c} \tilde{\mathcal{F}}_{c}} \operatorname{GRAD}_{c}(\mathbf{q}) \rrbracket_{\mathcal{E}_{c} \tilde{\mathcal{F}}_{c}},\right.
$$


which readily inherits the symmetry property of $\mathrm{H}_{\lambda}^{\varepsilon_{c} \tilde{\mathcal{F}}_{c}}$. Owing to the cellwise assembly of the discrete Hodge operator and the above commuting property, the left-hand side of (3.22) can be rewritten as

$$
\llbracket \operatorname{GRAD}(\mathbf{p}), \mathrm{H}_{\lambda}^{\varepsilon \tilde{\mathcal{F}}} \operatorname{GRAD}(\mathbf{q}) \rrbracket_{\mathcal{E} \tilde{\mathcal{F}}}=\sum_{c \in \mathrm{C}} \llbracket \mathrm{P}_{\mathcal{E}, c} \operatorname{GRAD}(\mathbf{p}), \mathrm{H}_{\lambda}^{\mathcal{E}_{c} \tilde{\mathcal{F}}_{c}} \mathrm{P}_{\mathcal{E}, c} \operatorname{GRAD}(\mathbf{q}) \rrbracket_{\mathcal{E}_{c} \tilde{\mathcal{F}}_{c}}=\sum_{c \in \mathrm{C}} \llbracket \mathrm{P}_{\mathcal{V}, c}(\mathbf{p}), \mathrm{P}_{\mathcal{V}, c}(\mathbf{q}) \rrbracket_{c}^{\mathrm{MFD}},
$$

so that the vertex-based scheme (3.22) fits the general form of nodal MFD schemes. We now verify that the bilinear form (3.23) verifies the two abstract properties identified in [8] for the convergence of nodal MFD schemes.

Proposition 3.11. Let $\mathrm{H}_{\lambda}^{\varepsilon_{c} \tilde{\mathcal{F}}_{c}}$ satisfy, for all $c \in \mathrm{C}$, the design properties $(\mathbf{A 1 v})$ and $(\mathbf{A 2 v})$. Then, the two properties stated in equations (5.14)-(5.15) in [8] hold for the bilinear form $\llbracket \cdot, \cdot \rrbracket_{c}^{\mathrm{MFD}}$.

Proof. Equation (5.14) in [8] is a direct consequence of (A1v). To verify equation (5.15) in [8], let $c \in \mathrm{C}$ and let $r \in \mathbb{P}_{1}(c)$. Then, for all $\mathbf{q} \in \mathcal{V}_{c}$, letting $\mathbf{R}_{\mathcal{V}_{c}}(r)$ collect the degrees of freedom of $r$ at local vertices, we obtain

$$
\begin{aligned}
\llbracket \mathbf{q}, \mathrm{R}_{\mathcal{V}_{c}}(r) \rrbracket_{c}^{\mathrm{MFD}} & =\llbracket \operatorname{GRAD}_{c}(\mathbf{q}), \mathrm{H}_{\lambda}^{\varepsilon_{c} \tilde{\mathcal{F}}_{c}} \operatorname{GRAD}_{c}\left(\operatorname{R}_{\mathcal{V}_{c}}(r)\right) \rrbracket_{\mathcal{E}_{c} \tilde{\mathcal{F}}_{c}}=\llbracket \operatorname{GRAD}_{c}(\mathbf{q}), \mathrm{H}_{\lambda}^{\varepsilon_{c} \tilde{\mathcal{F}}_{c}} \mathrm{R}_{\mathcal{E}_{c}}(\underline{\operatorname{grad}} r) \rrbracket_{\mathcal{E}_{c} \tilde{\mathcal{F}}_{c}} \\
& =\llbracket \operatorname{GRAD}_{c}(\mathbf{q}), \mathrm{R}_{\widetilde{\mathcal{F}}_{c}}(\underline{\underline{\operatorname{grad}}} r) \rrbracket_{\mathcal{E}_{c} \tilde{\mathcal{F}}_{c}}=\sum_{e \in \mathrm{E}_{c}} \sum_{v \in \mathrm{V}_{e}} \iota_{v, e} \mathbf{q}_{v}(\underline{\underline{\lambda}} \underline{\operatorname{grad}} r) \cdot \underline{\tilde{f}}_{c}(e) \\
& =\sum_{v \in \mathrm{V}_{c}} \mathbf{q}_{v}(\underline{\underline{\lambda}} \underline{\operatorname{grad}} r) \cdot\left(\sum_{e \in \mathrm{E}_{v} \cap \mathrm{E}_{c}} \iota_{v, e} \underline{\tilde{f}}_{c}(e)\right)
\end{aligned}
$$

where we have used (A2v) (since $\underline{\underline{\lambda}} \underline{\operatorname{grad}} r$ is constant in $c$ ) and the definition of $\mathrm{R}_{\widetilde{\mathcal{F}}_{c}}$. For each $v \in \mathrm{V}_{c}$, the set of faces of the polyhedron $c \cap \tilde{c}(v)$ consists of the dual faces $\tilde{f}_{c}(e)$ for all $e \in \mathrm{E}_{v} \cap \mathrm{E}_{c}$ and of subfaces of those faces of $c$ containing $v$. Let $\mathrm{F}_{v, c}$ collect such subfaces. For each $\hat{f} \in \mathrm{F}_{v, c}$, let $\underline{\hat{n}}_{\hat{f}, c}$ be the unit normal to $\hat{f}$ pointing outward $c$. Set $\underline{\hat{f}}:=\int_{\hat{f}} \underline{\hat{n}}_{\hat{f}, c}$. Then, it is easily verified that $\sum_{e \in \mathrm{E}_{v} \cap \mathrm{E}_{c}} \iota_{v, e} \underline{\tilde{f}}_{c}(e)=\sum_{\hat{f} \in \mathrm{F}_{v, c}} \underline{\hat{f}}$. As a result,

$$
\llbracket \mathbf{q}, \mathrm{R}_{\mathcal{V}_{c}}(r) \rrbracket_{c}^{\mathrm{MFD}}=\sum_{v \in \mathrm{V}_{c}} \sum_{\hat{f} \in \mathrm{F}_{v, c}} \mathbf{q}_{v}(\underline{\underline{\lambda}} \underline{\operatorname{grad}} r) \cdot \underline{\hat{f}},
$$

which is a specific form of equation (5.15) in [8] for a suitable integration formula on the faces of $c$.

\section{Analysis of VerteX-BASED schemes Using GRAdiEnt RECONSTRUCtion}

\subsection{Construction of a dual barycentric mesh}

A dual barycentric mesh is built from a barycentric subdivision of the primal mesh defined as follows. Let $\underline{x}_{e}$ and $\underline{x}_{f}$ denote, respectively, the barycenter of $e \in \mathrm{E}$ and $f \in \mathrm{F}$. We assume that the primal faces are planar and that they are star-shaped with respect to $\underline{x}_{f}$. Let $\underline{x}_{c}$ denote an arbitrary point in $c \in \mathrm{C}$ such that $c$ is star-shaped with respect to $\underline{x}_{c}$. For all $1 \leq k \leq 3$, given $(k+1)$ points $\left\{\underline{x}_{0}, \ldots, \underline{x}_{k}\right\}, \mathfrak{s}\left(\underline{x}_{0}, \ldots, \underline{x}_{k}\right)$ denotes the convex hull of these points (yielding, up to degenerate cases, a segment for $k=1$, a triangle for $k=2$, and a tetrahedron for $k=3$ ). For all $c \in \mathrm{C}$, a barycentric subdivision of $c$ consists of $4\left(\# \mathrm{E}_{c}\right)$ elementary subsimplices defined, for all $e \in \mathrm{E}_{c}$, as $\mathfrak{s}\left(\underline{x}_{v_{ \pm}}, \underline{x}_{e}, \underline{x}_{f_{ \pm}}, \underline{x}_{c}\right)$ for $v_{ \pm} \in \mathrm{V}_{e}$ and $f_{ \pm} \in \mathrm{F}_{e} \cap \mathrm{F}_{c}$; see Figure 4 left. The choice of the point $\underline{x}_{c}$ is the only free parameter in a barycentric subdivision; when $\underline{x}_{c}$ is the barycenter of $c$, the subdivision is called fully barycentric. Once a barycentric subdivision of the primal mesh is chosen, the resulting dual barycentric mesh is such that $\tilde{v}(c):=\underline{x}_{c}, \tilde{e}(f):=\cup_{c \in \mathrm{C}_{f}} \mathfrak{s}\left(\underline{x}_{f}, \underline{x}_{c}\right), \tilde{f}(e):=\cup_{f \in \mathrm{F}_{e}} \cup_{c \in \mathrm{C}_{f}} \mathfrak{s}\left(\underline{x}_{e}, \underline{x}_{f}, \underline{x}_{c}\right)$, and $\tilde{c}(v):=\cup_{e \in \mathrm{E}_{v}} \cup_{f \in \mathrm{F}_{e}} \cup_{c \in \mathrm{C}_{f}} \mathfrak{s}\left(\underline{x}_{v}, \underline{x}_{e}, \underline{x}_{f}, \underline{x}_{c}\right)$. With this construction, $\tilde{f}_{c}(e):=\cup_{f \in \mathrm{F}_{e} \cap \mathrm{F}_{c}} \mathfrak{s}\left(\underline{x}_{e}, \underline{x}_{f}, \underline{x}_{c}\right)$ is the union

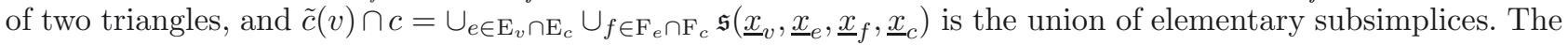



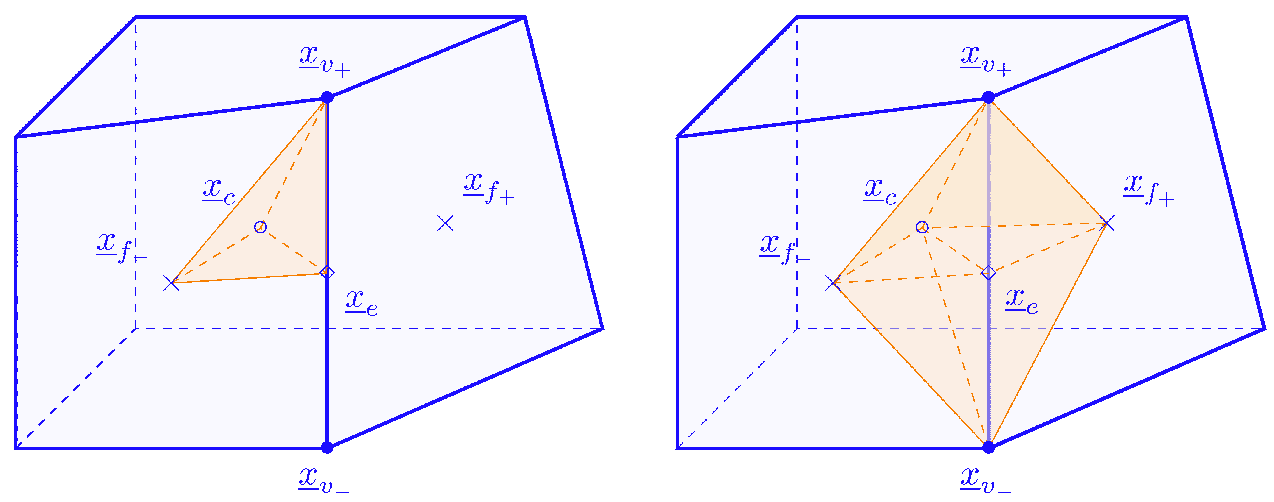

FiguRE 4. Left: barycentric subdivision of a primal cell with an elementary subsimplex highlighted $\left(\underline{x}_{c}\right.$ is not necessarily the barycenter of $\left.c\right)$; Right: elementary subvolume considered for the gradient reconstruction in the Discrete Geometric Approach.

elementary subsimplices allow one to build the simplicial submesh considered in assumption (M1). Following Codecasa et al. $[13,14]$, since primal faces are planar, a dual barycentric mesh satisfies, for all $c \in \mathrm{C}$,

$$
\sum_{e \in \mathrm{E}_{c}} \underline{\tilde{f}}_{c}(e) \otimes \underline{e}=\sum_{e \in \mathrm{E}_{c}} \underline{e} \otimes \underline{\tilde{f}}_{c}(e)=|c| \underline{\underline{\mathrm{Id}}},
$$

where

$$
\underline{e}:=\int_{e} \underline{\tau}_{e}, \quad \underline{\tilde{f}}_{c}(e):=\int_{\tilde{f}_{c}(e)} \underline{\nu}_{\tilde{f}(e)} .
$$

\subsection{Local gradient reconstruction and local discrete Hodge operator}

For all primal cell $c \in \mathrm{C}$, we consider a family of $\# \mathrm{E}_{c}$ linearly independent functions $\underline{\ell}_{e, c}: c \rightarrow \mathbb{R}^{3}$ spanning a finite-dimensional space of vector-valued functions denoted $P_{\mathcal{E}}(c) \subset\left[L^{2}(c)\right]^{3}$. We introduce the local lifting operator

$$
\underline{\mathrm{L}}_{\mathcal{E}_{c}}: \mathcal{E}_{c} \ni \mathbf{g} \longmapsto \underline{\mathrm{L}}_{\mathcal{E}_{c}}(\mathrm{~g}):=\sum_{e \in \mathrm{E}_{c}} \mathrm{~g}_{e} \underline{\ell}_{e, c}(\underline{x}) \in P_{\mathcal{E}}(c) .
$$

Functions in $P_{\mathcal{E}}(c)$ are assumed to be sufficiently smooth so that the local reduction operator $\mathrm{R}_{\mathcal{E}_{c}}$ can act on them. Typically, functions in $P_{\mathcal{E}}(c)$ are piecewise polynomials on a submesh of $c$ composed of elementary subsimplices or aggregations thereof. We stress that the tangential component of these functions is not necessarily continuous on the edges of this submesh lying inside $c$, so that, in general, $P_{\mathcal{E}}(c) \not \subset H(\underline{\text { curl }} ; c)$. The requirements on the local reconstruction functions $\left\{\underline{\ell}_{e, c}\right\}_{e \in \mathrm{E}_{c}}$ are (see Codecasa and Trevisan [15] except for (R2v)):

(R1v) [Unisolvence]. For all $e, e^{\prime} \in \mathrm{E}_{c}, \int_{e^{\prime}} \underline{\ell}_{e, c}(\underline{x}) \cdot \underline{\tau}_{e^{\prime}}=\delta_{e, e^{\prime}}$.

(R2v) [Stability]. There is $\eta>0$ such that, for all $\mathbf{g} \in \mathcal{E}_{c}, \eta\|\mathbf{g}\|_{2, \mathcal{E}_{c}}^{2} \leq\left\|\underline{\mathbf{L}}_{\mathcal{E}_{c}}(\mathbf{g})\right\|_{L^{2}(c)^{3}}^{2} \leq \eta^{-1}\|\mathbf{g}\|_{2, \mathcal{E}_{c}}^{2}$.

(R3v) [Partition of unity]. For all $\underline{x} \in c, \sum_{e \in \mathrm{E}_{c}} \underline{\ell}_{e, c}(\underline{x}) \otimes \underline{e}=\underline{\underline{\mathrm{Id}}}$.

(R4v) [Geometric consistency]. For all $e \in \mathrm{E}_{c}, \int_{c} \underline{\ell}_{e, c}(\underline{x})=\underline{\tilde{f}}_{c}(e)$.

As shown by Codecasa et al. $[13,14]$, the unisolvence property $(\mathbf{R} 1 \mathbf{v})$ is equivalent to

$$
\mathrm{R}_{\mathcal{E}_{c}} \underline{\underline{L}}_{\mathcal{E}_{c}}=\mathrm{Id}_{\mathcal{E}_{c}} .
$$

Moreover, the partition of unity property (R3v) is equivalent to the fact that, for all $\underline{G}$ constant in $c$,

$$
\underline{\mathrm{L}}_{\mathcal{E}_{c}} \mathrm{R}_{\mathcal{E}_{c}}(\underline{G})=\underline{G} .
$$

Finally, we observe that (4.1) is a necessary condition for (R3v) and (R4v) to hold simultaneously. 
Definition 4.1. The local discrete Hodge operator is specified by its entries such that, for all $e, e^{\prime} \in \mathrm{E}_{c}$,

$$
\left(\mathrm{H}_{\lambda}^{\varepsilon_{c} \tilde{\mathcal{F}}_{c}}\right)_{e, e^{\prime}}:=\int_{c} \underline{\ell}_{e, c}(\underline{x}) \cdot \underline{\underline{\lambda}} \underline{e}_{e^{\prime}, c}(\underline{x})
$$

Owing to this definition, it is easily seen that

$$
\forall \mathbf{g}_{1}, \mathbf{g}_{2} \in \mathcal{E}_{c}, \quad \llbracket \mathbf{g}_{1}, \mathrm{H}_{\lambda}^{\mathcal{E}_{c} \tilde{\mathcal{F}}_{c}} \mathbf{g}_{2} \rrbracket_{\mathcal{E}_{c} \tilde{\mathcal{F}}_{c}}=\int_{c} \underline{\mathrm{L}}_{\mathcal{E}_{c}}\left(\mathbf{g}_{1}\right) \cdot \underline{\underline{\lambda}} \underline{\underline{\mathrm{L}}} \underline{\mathcal{E}}_{c}\left(\mathbf{g}_{2}\right) .
$$

Proposition 4.2. Let $\mathbf{H}_{\lambda}^{\varepsilon_{c} \tilde{\mathcal{F}}_{c}}$ be defined by (4.6). Assume (R1v)-(R4v). Then, (A1v) and (A2v) hold.

Proof. It is readily verified that $\mathrm{H}_{\lambda}^{\mathcal{E}_{c} \tilde{\mathcal{F}}_{c}}$ is SPD. The stability property in (A1v) results from (R2v) and (4.7). Finally, let $\underline{G}$ be constant in $c$. Then, we infer, for all $e \in \mathrm{E}_{c}$,

$$
\left(\mathrm{H}_{\lambda}^{\mathcal{E}_{c} \tilde{\mathcal{F}}_{c}} \mathrm{R}_{\mathcal{E}_{c}}(\underline{G})\right)_{e}=\int_{c} \underline{\ell}_{e, c}(\underline{x}) \cdot \underline{\underline{\lambda}} \underline{\underline{E}}_{\mathcal{E}_{c}} \mathrm{R}_{\mathcal{E}_{c}}(\underline{G})=\int_{c} \underline{\ell}_{e, c}(\underline{x}) \cdot \underline{\underline{\lambda}} \underline{G}=\underline{\tilde{f}}_{c}(e) \cdot \underline{\underline{\lambda}} \underline{G}=\left(\mathrm{R}_{\tilde{\mathcal{F}}_{c}}(\underline{\underline{\lambda}} \underline{G})\right)_{e},
$$

using (4.5) and (R4v) and the fact that $\underline{\underline{\lambda}}$ is constant in $c$. This yields $(\mathbf{A 2 \mathbf { v }})$.

\subsection{Examples}

\subsubsection{Diagonal discrete Hodge operator on Cartesian meshes}

Consider a Cartesian mesh. Let $c \in \mathrm{C}$. Consider a fully barycentric subdivision of $c$; see Section 4.1. For all

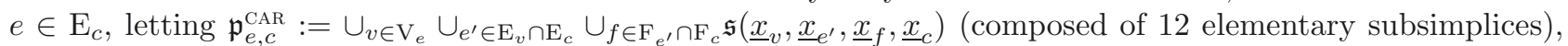
we set $\underline{\ell}_{e, c}^{\mathrm{CAR}}(\underline{x}):=|e|^{-1} \underline{\tau}_{e}$ if $\underline{x} \in \mathfrak{p}_{e, c}^{\mathrm{CAR}}$ and $\underline{\ell}_{e, c}^{\mathrm{CAR}}(\underline{x}):=\underline{0}$ otherwise. Then, properties (R1v)-(R4v) are easily verified. Moreover, for isotropic conductivity, the local discrete Hodge operator defined by (4.6) is diagonal and corresponds to $(2.14)$.

\subsubsection{Whitney forms (edge finite elements) on tetrahedral meshes}

Consider a tetrahedral mesh. Let $c \in \mathrm{C}$. For all $e \in \mathrm{E}_{c}$, set $\underline{\ell}_{e, c}^{\mathrm{WHI}}(\underline{x}):=\left.\underline{\varphi}_{e}^{\mathrm{NED}}\right|_{c}(\underline{x})$, where $\underline{\varphi}_{e}^{\mathrm{NED}}$ is the lowestorder Nédélec edge finite element function attached to $e$. Then, properties $(\overline{\mathbf{R}} \mathbf{1} \mathbf{v})-(\mathbf{R} 4 \mathbf{v})$ are easily verified. The vertex-based scheme (2.9) then coincides with the classical first-order Lagrange finite element approximation on the primal mesh (up to a quadrature on the source term); see Bossavit [7], no. 5.

\subsubsection{Discrete Geometric Approach on polyhedral meshes}

Consider a polyhedral mesh. Let $c \in \mathrm{C}$. Consider a barycentric subdivision of $c$. For all $e \in \mathrm{E}_{c}$, letting $\mathfrak{p}_{e, c}^{\mathrm{DGA}}:=\cup_{v \in \mathrm{V}_{e}} \cup_{f \in \mathrm{F}_{e} \cap \mathrm{F}_{c}} \mathfrak{s}\left(\underline{x}_{v}, \underline{x}_{e}, \underline{x}_{f}, \underline{x}_{c}\right)$ (composed of 4 elementary subsimplices, see Fig. 4 right), we set, following Codecasa et al. $[13,14]$,

$$
\forall e^{\prime} \in \mathrm{E}_{c},\left.\quad \underline{\ell}_{e, c}^{\mathrm{DGA}}\right|_{\mathfrak{p}_{e^{\prime}, c}^{\mathrm{DGA}}}:=\frac{\underline{\tilde{f}}_{c}(e)}{\underline{\tilde{f}}_{c}(e) \cdot \underline{e}} \delta_{e, e^{\prime}}+\left(\underline{\underline{\mathrm{Id}}}-\frac{\underline{\tilde{f}}_{c}\left(e^{\prime}\right) \otimes \underline{e}^{\prime}}{\underline{\tilde{f}}_{c}\left(e^{\prime}\right) \cdot \underline{e}^{\prime}}\right) \frac{\underline{\tilde{f}}_{c}(e)}{|c|},
$$

so that $\underline{\ell}_{e, c}^{\mathrm{DGA}}$ is piecewise constant on the submesh $\left\{\mathfrak{p}_{e^{\prime}, c}^{\mathrm{DGA}}\right\}_{e^{\prime} \in \mathrm{E}_{c}}$ of $c$. Properties (R1v), (R3v), and (R4v) are shown in [15], while property $(\mathbf{R 2 v})$ is readily verified using mesh regularity (in particular, $\left.|e|\left|\tilde{f}_{c}(e)\right| \lesssim \underline{e} \cdot \underline{\tilde{f}}_{c}(e)\right)$.

\subsection{Global gradient reconstruction and discrete Hodge operator}

The global gradient reconstruction operator $\underline{\underline{L}}_{\mathcal{E}}: \mathcal{E} \rightarrow P_{\mathcal{E}}(\mathrm{C})$ is defined such that, for all $\mathbf{g} \in \mathcal{E}$,

$$
\forall c \in \mathrm{C},\left.\quad \underline{\mathrm{L}}_{\mathcal{E}}(\mathrm{g})\right|_{c}:=\underline{\mathrm{L}}_{\mathcal{E}_{c}}\left(\mathrm{P}_{\mathcal{E}, c}(\mathrm{~g})\right) .
$$

This operator maps onto the broken polynomial space $P_{\mathcal{E}}(\mathrm{C}):=\left\{\underline{g} \in\left[L^{2}(c)\right]^{3} ; \forall c \in \mathrm{C},\left.\underline{g}\right|_{c} \in P_{\mathcal{E}}(c)\right\}$ which is, in general, nonconforming, that is, $P_{\mathcal{E}}(\mathrm{C}) \not \subset H(\underline{\text { curl }}, \Omega)$. Owing to $(\overline{\mathbf{R} 1 \mathbf{v})})$, the tangential component of $\underline{\mathrm{L}}_{\mathcal{E}}(\mathrm{g})$ is 
single-valued on all primal edges $e \in \mathrm{E}$, so that $\mathrm{R}_{\mathcal{E}}$ can act on $\underline{\mathrm{L}}_{\mathcal{E}}(\mathrm{g})$, and, as a consequence of $(4.4), \mathrm{R}_{\mathcal{E}} \underline{\mathrm{L}}_{\mathcal{E}}=\mathrm{Id}_{\mathcal{E}}$. Moreover, as a consequence of $(\mathbf{R} 2 \mathbf{v})$, we infer, for all $\mathbf{g} \in \mathcal{E}$,

$$
\eta\|\mathbf{g}\|_{2, \mathcal{E}}^{2} \leq\left\|\underline{\underline{L}}_{\mathcal{E}}(\mathbf{g})\right\|_{L^{2}(\Omega)^{3}}^{2} \leq \eta^{-1}\|\mathbf{g}\|_{2, \mathcal{E}}^{2}
$$

A consequence of (4.7) and the cellwise assembly of the discrete Hodge operator is

$$
\forall \mathbf{g}_{1}, \mathbf{g}_{2} \in \mathcal{E}, \quad \llbracket \mathbf{g}_{1}, \mathrm{H}_{\lambda}^{\mathcal{E} \tilde{\mathcal{F}}} \mathbf{g}_{2} \rrbracket_{\mathcal{E} \tilde{\mathcal{F}}}=\int_{\Omega} \underline{\underline{\mathrm{L}}}\left(\mathbf{g}_{1}\right) \cdot \underline{\underline{\lambda}} \underline{\underline{\mathcal{E}}}\left(\mathbf{g}_{2}\right)
$$

The vertex-based scheme (3.22) can then be recast into the functional form: Find $\mathbf{p} \in \mathcal{V}$ such that

$$
\int_{\Omega} \underline{\mathrm{L}}_{\mathcal{E}}(\operatorname{GRAD}(\mathbf{p})) \cdot \underline{\underline{\lambda}} \underline{\mathrm{L}}_{\mathcal{E}}(\operatorname{GRAD}(\mathbf{q}))=\int_{\Omega} s \mathrm{~L}_{\mathcal{V}}^{0}(\mathbf{q}), \quad \forall \mathbf{q} \in \mathcal{V}
$$

recalling the operator $L_{\mathcal{V}}^{0}$ defined in Section 3.3.2.

Proposition 4.3. The nonconforming approximation map $\underline{\mathrm{A}}_{\mathcal{E}}:=\underline{\mathrm{L}}_{\mathcal{E}} \mathrm{R}_{\mathcal{E}}: S_{\mathcal{E}}(\Omega) \rightarrow P_{\mathcal{E}}(\mathrm{C})$ is a projector, it satisfies, for all piecewise constant $\underline{G}$ on the primal mesh,

$$
\underline{\mathrm{A}}_{\mathcal{E}}(\underline{G})=\underline{G},
$$

and, for all $\underline{g} \in\left[H^{1}(\mathrm{C})\right]^{3} \cap S_{\mathcal{E}}(\Omega)$ which is curl-free, assuming that the barycentric subdivision satisfies (M1),

$$
\left\|\underline{g}-\underline{\mathrm{A}}_{\mathcal{E}}(\underline{g})\right\|_{L^{2}(\Omega)^{3}} \lesssim h_{\mathrm{M}}\|\underline{g}\|_{H^{1}(\mathrm{C})^{3}} .
$$

Proof. The map $\underline{\mathrm{A}}_{\mathcal{E}}$ is a projector since $\underline{\mathrm{A}}_{\mathcal{E}} \underline{\mathrm{A}}_{\mathcal{E}}=\underline{\mathrm{L}}_{\mathcal{E}}\left(\mathrm{R}_{\mathcal{E}} \underline{\mathrm{L}}_{\mathcal{E}}\right) \mathrm{R}_{\mathcal{E}}=\underline{\mathrm{L}}_{\mathcal{E}} \mathrm{R}_{\mathcal{E}}=\underline{\mathrm{A}}_{\mathcal{E}}$. Additionally, property (4.13) stems from (4.5). Finally, let $\underline{g} \in\left[H^{1}(\mathrm{C})\right]^{3} \cap S_{\mathcal{E}}(\Omega)$ be curl-free. Let $\underline{G}$ be the $L^{2}$-orthogonal projection of $\underline{g}$ onto $\left[\mathbb{P}_{0}(\mathrm{C})\right]^{3}$. Using the triangle inequality and (4.13), we obtain

$$
\left\|\underline{g}-\underline{\mathrm{A}}_{\mathcal{E}}(\underline{g})\right\|_{L^{2}(\Omega)^{3}} \leq\|\underline{g}-\underline{G}\|_{L^{2}(\Omega)^{3}}+\|\underline{\mathrm{A}} \mathcal{E}(\underline{g}-\underline{G})\|_{L^{2}(\Omega)^{3}} .
$$

Clearly, $\|\underline{g}-\underline{G}\|_{L^{2}(\Omega)^{3}} \lesssim h_{\mathrm{M}}\|\underline{g}\|_{H^{1}(\mathrm{C})^{3}}$. Moreover, owing to the upper bound in (4.10), we infer $\left\|\underline{\mathrm{A}}_{\mathcal{E}}(\underline{g}-\underline{G})\right\|_{L^{2}(\Omega)^{3}}^{2} \lesssim$ $\sum_{c \in \mathrm{C}} \sum_{e \in \mathrm{E}_{c}} \frac{\left|\tilde{f}_{c}(e)\right|}{|e|}\left|T_{1, e}\right|^{2}$, where $T_{1, e}=\int_{e}(\underline{g}-\underline{G}) \cdot \underline{\tau}_{e}$ has been bounded in the proof of Theorem 3.7.

Corollary 4.4 (Error estimate on reconstructed gradient and flux). Let g be the exact gradient and $\phi$ the exact

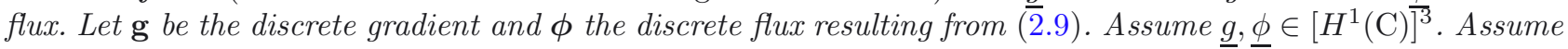
that the barycentric subdivision satisfies $(\mathrm{M} 1)$. Then, letting $\underline{\mathrm{L}}_{\widetilde{\mathcal{F}}}(\phi):=-\underline{\underline{\lambda}} \underline{\mathrm{L}}_{\mathcal{E}}(\mathrm{g})$,

$$
\left\|\underline{g}-\underline{\mathrm{L}}_{\mathcal{E}}(\mathrm{g})\right\|_{\lambda}=\left\|\underline{\phi}-\underline{\mathrm{L}}_{\widetilde{\mathcal{F}}}(\phi)\right\|_{\lambda^{-1}} \lesssim h_{\mathrm{M}}\left(\lambda_{\sharp}^{1 / 2}\|\underline{g}\|_{H^{1}(\mathrm{C})^{3}}+\lambda_{b}^{-1 / 2}\|\underline{\phi}\|_{H^{1}(\mathrm{C})^{3}}\right) .
$$

Proof. Since $\underline{g}-\underline{\mathrm{L}}_{\mathcal{E}}(\mathrm{g})=\left(\underline{g}-\underline{\mathrm{A}}_{\mathcal{E}}(\underline{g})\right)+\underline{\mathrm{L}}_{\mathcal{E}}\left(\mathrm{R}_{\mathcal{E}}(\underline{g})-\mathrm{g}\right)$, we infer using (4.11),

$$
\left\|\underline{g}-\underline{\mathrm{L}}_{\mathcal{E}}(\mathrm{g})\right\|_{\lambda} \leq\left\|\underline{g}-\underline{\mathrm{A}}_{\mathcal{E}}(\underline{g})\right\|_{\lambda}+\left\|\mathrm{R}_{\mathcal{E}}(\underline{g})-\mathbf{g}\right\|_{\lambda} .
$$

The first term on the right-hand side is estimated using (4.14), while the second term has been estimated in Theorem 3.7. The estimate on the flux is straightforward owing to the definition of $\underline{L}_{\tilde{\mathcal{F}}}$. 


\subsection{Link with approximate gradient schemes}

Approximate Gradient Schemes have been introduced recently by Eymard et al. [23]. We first observe that (4.12) matches the general form of equation (1.2) in [23] with the potential reconstruction $\operatorname{L}_{\mathcal{V}}^{0}(\mathbf{p}) \in L^{2}(\Omega)$ and the gradient reconstruction $\underline{\mathrm{L}}_{\mathcal{E}}(\operatorname{GRAD}(\mathbf{p})) \in\left[L^{2}(\Omega)\right]^{3}$. The convergence analysis of Approximate Gradient Schemes hinges on three properties (we adopt the terminology of [23]): the coercivity, stating that, there is $C$ such that, uniformly in $h_{\mathrm{M}}$,

$$
\max _{\mathbf{q} \in \mathcal{V} \backslash\{0\}} \frac{\left\|\mathrm{L}_{\mathcal{V}}^{0}(\mathbf{q})\right\|_{L^{2}(\Omega)}}{\left\|\underline{\underline{L}}_{\mathcal{E}}(\operatorname{GRAD}(\mathbf{q}))\right\|_{L^{2}(\Omega)^{3}}} \leq C,
$$

the strong consistency, stating that, for all $\varphi \in H_{0}^{1}(\Omega)$,

$$
S_{\mathrm{M}}(\varphi):=\min _{\mathbf{q} \in \mathcal{V}}\left\{\left\|\mathrm{L}_{\mathcal{V}}^{0}(\mathbf{q})-\varphi\right\|_{L^{2}(\Omega)}+\left\|\underline{\mathrm{L}}_{\mathcal{E}}(\operatorname{GRAD}(\mathbf{q}))-\underline{\operatorname{grad}} \varphi\right\|_{L^{2}(\Omega)^{3}}\right\} \rightarrow 0 \quad \text { as } h_{\mathrm{M}} \rightarrow 0,
$$

and the dual consistency (or conformity), stating that, for all $\underline{\psi} \in H(\operatorname{div} ; \Omega)$,

$$
W_{\mathrm{M}}(\underline{\psi}):=\max _{\mathbf{q} \in \mathcal{V} \backslash\{0\}} \frac{1}{\left\|\underline{\underline{L}}_{\mathcal{E}}(\operatorname{GRAD}(\mathbf{q}))\right\|_{L^{2}(\Omega)^{3}}}\left|\int_{\Omega}\left\{\underline{\mathrm{L}}_{\mathcal{E}}(\operatorname{GRAD}(\mathbf{q})) \cdot \underline{\psi}+\mathrm{L}_{\mathcal{V}}^{0}(\mathbf{q}) \operatorname{div}(\underline{\psi})\right\}\right| \rightarrow 0 \quad \text { as } h_{\mathrm{M}} \rightarrow 0 .
$$

Proposition 4.5. Let $\mathbf{H}_{\lambda}^{\tilde{\mathcal{F}}}$ be defined by (4.11) with local reconstruction functions satisfying (R1v) $-(\mathbf{R 4 v})$. Then, (4.16), (4.17), and (4.18) hold with the first-order convergence rates $S_{\mathrm{M}}(\varphi) \lesssim h_{\mathrm{M}}\|\varphi\|_{H^{1}(\Omega)}$ and $W_{\mathrm{M}}(\underline{\psi}) \lesssim$ $\left(\lambda_{\sharp} / \lambda_{b}\right) h_{\mathrm{M}}\|\underline{\psi}\|_{H^{1}(\Omega)^{3}}$.

Proof. To prove (4.16), we use the discrete Poincaré inequality (3.19) and the lower bound in (4.10) yielding, for all $\mathbf{q} \in \mathcal{V}$,

$$
\left\|\mathrm{L}_{\mathcal{V}}^{0}(\mathbf{q})\right\|_{L^{2}(\Omega)}=\|\mathbf{q}\|_{2, \mathcal{V}} \leq C_{2,2}\|\operatorname{GRAD}(\mathbf{q})\|_{2, \mathcal{E}} \leq C_{2,2} \eta^{-1 / 2}\left\|\underline{\mathrm{L}}_{\mathcal{E}}(\operatorname{GRAD}(\mathbf{q}))\right\|_{L^{2}(\Omega)^{3}} .
$$

To prove (4.17), it suffices by density (see Lemma 2.5 in [23]) to consider $\varphi \in C_{0}^{\infty}(\Omega)$. We take $\mathbf{q}=\mathrm{R}_{\mathcal{V}}(\varphi)$ so that, owing to the commuting property of Lemma 2.1,

$$
\left.\underline{\mathrm{L}}_{\mathcal{E}}(\operatorname{GRAD}(\mathbf{q}))=\underline{\mathrm{L}}_{\mathcal{E}}(\operatorname{GRAD}(\operatorname{R} \mathcal{V}(\varphi)))=\underline{\mathrm{L}}_{\mathcal{E}} \operatorname{R}_{\mathcal{E}}(\underline{\operatorname{grad}} \varphi)=\underline{\mathrm{A}}_{\mathcal{E}} \underline{\operatorname{grad}} \varphi\right),
$$

whence we infer $\left\|\underline{L}_{\mathcal{E}}(\operatorname{GRAD}(\mathbf{q}))-\underline{\operatorname{grad}} \varphi\right\|_{L^{2}(\Omega)^{3}} \lesssim h_{\mathrm{M}}\|\varphi\|_{H^{1}(\Omega)}$ owing to Proposition 4.3. Moreover, the definition of $\mathrm{L}_{\mathcal{V}}^{0}$ yields $\left\|\mathrm{L}_{\mathcal{V}}^{0} \mathrm{R}_{\mathcal{V}}(\varphi)-\varphi\right\|_{L^{2}(\Omega)} \lesssim h_{\mathrm{M}}\|\varphi\|_{H^{1}(\Omega)}$, so that the same bound holds on $S_{\mathrm{M}}(\varphi)$. To prove (4.18), it suffices by density (see Lemma 2.5 in $[23]$ ) to consider $\underline{\psi} \in\left[C^{1}(\bar{\Omega})\right]^{3}$. We set $\underline{g}:=-\underline{\underline{\lambda}}^{-1} \underline{\psi}$. Then, for all $\mathbf{q} \in \mathcal{V}$, we first observe that, owing to the definition of $\mathbf{L}_{\mathcal{V}}^{0}$ and the commuting and adjunction properties of Lemmata 2.1 and 2.2 , respectively, there holds

$$
\int_{\Omega} \mathrm{L}_{\mathcal{V}}^{0}(\mathbf{q}) \operatorname{div}(\underline{\psi})=\llbracket \mathbf{q}, \mathrm{R}_{\widetilde{\mathcal{C}}}(\operatorname{div}(\underline{\psi})) \rrbracket_{\mathcal{V} \tilde{\mathcal{C}}}=\llbracket \mathbf{q}, \widetilde{\operatorname{DIV}}\left(\mathrm{R}_{\tilde{\mathcal{F}}}(\underline{\psi})\right) \rrbracket_{\mathcal{V} \tilde{\mathcal{C}}}=-\llbracket \operatorname{GRAD}(\mathbf{q}), \mathrm{R}_{\tilde{\mathcal{F}}}(\underline{\psi}) \rrbracket_{\mathcal{E} \tilde{\mathcal{F}}} .
$$

Hence,

$$
\begin{aligned}
\int_{\Omega}\left\{\underline{\mathrm{L}}_{\mathcal{E}}(\operatorname{GRAD}(\mathbf{q})) \cdot \underline{\psi}+\mathrm{L}_{\mathcal{V}}^{0}(\mathbf{q}) \operatorname{div}(\underline{\psi})\right\} & =\int_{\Omega} \underline{\mathrm{L}}_{\mathcal{E}}(\operatorname{GRAD}(\mathbf{q})) \cdot \underline{\psi}-\llbracket \operatorname{GRAD}(\mathbf{q}), \mathrm{R}_{\tilde{\mathcal{F}}}(\underline{\psi}) \rrbracket_{\mathcal{E} \tilde{\mathcal{F}}} \\
& =\int_{\Omega} \underline{\mathrm{L}}_{\mathcal{E}}(\operatorname{GRAD}(\mathbf{q})) \cdot \underline{\underline{\lambda}}(\underline{\mathrm{A}} \underline{\mathcal{E}}(\underline{g})-\underline{g})-\left[\operatorname{GRAD}(\mathbf{q}),\lfloor\lambda, \operatorname{R}\rceil(\underline{g}) \rrbracket_{\mathcal{E} \tilde{\mathcal{F}}}=: T_{1}+T_{2},\right.
\end{aligned}
$$

since

$$
\int_{\Omega} \underline{\mathrm{L}}_{\mathcal{E}}(\operatorname{GRAD}(\mathbf{q})) \cdot \underline{\underline{\lambda}} \underline{\mathrm{A}}_{\mathcal{E}}(\underline{g})=\int_{\Omega} \underline{\mathrm{L}}_{\mathcal{E}}(\operatorname{GRAD}(\mathbf{q})) \cdot \underline{\underline{\lambda}} \underline{\mathrm{L}}_{\mathcal{E}}\left(\mathrm{R}_{\mathcal{E}}(\underline{g})\right)=\llbracket \operatorname{GRAD}(\mathbf{q}), \mathrm{H}_{\lambda}^{\mathcal{E} \tilde{\mathcal{F}}} \mathrm{R}_{\mathcal{E}}(\underline{g}) \rrbracket_{\mathcal{E} \tilde{\mathcal{F}}}
$$

Then, $\left|T_{1}\right| /\left\|\underline{\mathrm{L}}_{\mathcal{E}}(\operatorname{GRAD}(\mathbf{q}))\right\|_{L^{2}(\Omega)^{3}} \leq \lambda_{\sharp}\left\|\underline{g}-\underline{\mathrm{A}}_{\mathcal{E}}(\underline{g})\right\|_{L^{2}(\Omega)^{3}} \lesssim \lambda_{\sharp} h_{\mathrm{M}}\|\underline{g}\|_{H^{1}(\mathrm{C})^{3}} \leq\left(\lambda_{\sharp} / \lambda_{b}\right) h_{\mathrm{M}}\|\underline{\psi}\|_{H^{1}(\Omega)^{3}}$ owing to Proposition 4.3. Moreover, since the upper bound in (3.11) combined with the lower bound in (4.10) implies $\|\mathbf{g}\|_{\lambda} \leq \lambda_{\sharp}^{1 / 2} \eta^{-1}\left\|\underline{\underline{L}}_{\mathcal{E}}(\mathbf{g})\right\|_{L^{2}(\Omega)^{3}}$ for all $\mathbf{g} \in \mathcal{E}$, we obtain $\left|T_{2}\right| \leq \lambda_{\sharp}^{1 / 2} \eta^{-1}\left\|_{\underline{\mathcal{E}}}(\operatorname{GRAD}(\mathbf{q}))\right\|_{L^{2}(\Omega)^{3}}\|\lfloor\lambda, \operatorname{R}\rceil(\underline{g})\|_{\lambda^{-1}}$, and the last factor has been estimated in Theorem 3.7, yielding the desired bound on $W_{\mathrm{M}}(\underline{\psi})$. 


\section{6. $L^{2}$-error estimate}

For simplicity, we assume in this section that $\underline{\underline{\lambda}}$ is the identity tensor. Moreover, we assume a fully barycentric subdivision. The proof of our $L^{2}$-error estimate hinges on the existence of a conforming potential reconstruction with suitable properties. The proof of Lemma 4.6 is postponed to Appendix B.

Lemma 4.6. Assume a fully barycentric subdivision satisfying (M1) and planar primal faces. Then, there exists a potential reconstruction operator $\mathbf{L}_{\mathcal{V}}^{\text {conf }}$ built from local operators $\mathbf{L}_{\mathcal{V}_{c}}^{\text {conf }}$ by setting $\left.\mathbf{L}_{\mathcal{V}}^{\text {conf }}\right|_{c}:=\operatorname{L}_{\mathcal{V}_{c}}^{\text {conf }}$ for all $c \in \mathrm{C}$ and satisfying the following properties:

(i) (Conformity) $\mathfrak{L}_{\mathcal{V}}^{\text {conf }}: \mathcal{V} \rightarrow H_{0}^{1}(\Omega)$;

(ii) (Unisolvence) $\mathrm{R}_{\mathcal{V}} \mathrm{L}_{\mathcal{V}}^{\text {conf }}=\mathrm{Id}_{\mathcal{V}}$;

(iii) (Stability) For all $c \in \mathrm{C}$ and all $\mathbf{p} \in \mathcal{V}_{c},\left\|\mathrm{~L}_{\mathcal{V}_{c}}(\mathbf{p})\right\|_{L^{2}(c)} \lesssim\|\mathbf{p}\|_{2, \mathcal{V}_{c}}\left(\right.$ where $\left.\|\mathbf{p}\|_{2, \mathcal{V}_{c}}^{2}=\sum_{v \in \mathrm{V}_{c}}\left|c \cap \tilde{c}(v) \| \mathbf{p}_{v}\right|^{2}\right)$ and $\left\|\operatorname{grad}\left(\operatorname{L}_{\mathcal{V}_{c}}^{\operatorname{conf}}(\mathbf{p})\right)\right\|_{L^{2}(c)^{3}} \lesssim\|\operatorname{GRAD}(\mathbf{p})\|_{2, \mathcal{E}_{c}} ;$

(iv) $\left(\mathbb{P}_{1}\right.$-consistency) For all $c \in \mathrm{C}$ and all $P \in \mathbb{P}_{1}(c)$, there holds $\mathrm{A}_{\mathcal{V}_{c}}^{\text {conf }}(P)=P$ with $A_{\mathcal{V}_{c}}^{\text {conf }}:=\mathrm{L}_{\mathcal{V}_{c}}^{\text {conf }} \mathrm{R}_{\mathcal{V}_{c}}$;

(v) (Geometric consistency) For all $\mathbf{p} \in \mathcal{V}, \mathbf{L}_{\mathcal{V}}^{\operatorname{conf}}(\mathbf{p})$ and $\mathbf{L}_{\mathcal{V}}^{0}(\mathbf{p})$ (defined in Section 3.3.2) have the same mean-value on primal cells, and the same property holds for $\underline{\operatorname{grad}}\left(\mathrm{L}_{\mathcal{V}}^{\operatorname{conf}}(\mathbf{p})\right)$ and $\underline{\mathrm{L}}_{\mathcal{E}}(\operatorname{GRAD}(\mathbf{p}))$.

Proposition 4.7. Assume a conforming potential reconstruction with properties of Lemma 4.6. Then, there holds, for all $\zeta \in H^{2}(\Omega),\left\|\underline{\operatorname{grad}}(\zeta)-\underline{\operatorname{grad}}\left(\mathrm{A}_{\mathcal{V}}^{\operatorname{conf}}(\zeta)\right)\right\|_{L^{2}(\Omega)^{3}} \lesssim h_{\mathrm{M}}\|\zeta\|_{H^{2}(\Omega)}$.

Proof. The estimate is proved locally on each primal cell using Lemma 4.6 (iii) and (iv). Adding and subtracting the gradient of the $L^{2}$-orthogonal projection of $\zeta$ on $\mathbb{P}_{1}(c)$, say $\pi_{1, c}(\zeta)$, using the triangle inequality and local stability yields $\left\|\underline{\operatorname{grad}}(\zeta)-\underline{\operatorname{grad}}\left(\mathrm{A}_{\mathcal{V}}^{\operatorname{conf}}(\zeta)\right)\right\|_{L^{2}(c)^{3}} \lesssim h_{c}\|\underline{\operatorname{grad}}(\zeta)\|_{H^{1}(c)^{3}}+\left\|\mathrm{R}_{\mathcal{E}_{c}}\left(\underline{\operatorname{grad}}\left(\zeta-\pi_{1, c}(\zeta)\right)\right)\right\|_{2, \mathcal{E}_{c}}$ and the last term is bounded by $h_{c}\|\underline{\operatorname{grad}(\zeta)}\|_{H^{1}(c)^{3}}$ as in the proof of Theorem 3.7.

We can now turn to the main result of this section.

Theorem 4.8. Assume that the model problem (1.1) with $\underline{\underline{\lambda}}=\underline{\underline{\mathrm{Id}}}$ has elliptic regularity. Assume $s \in H^{1}(\Omega)$. Let $p$ be the exact potential and let $\mathbf{p}$ be the discrete potential resulting from (2.9). Assume a fully barycentric subdivision satisfying (M1) and planar primal faces. Then,

$$
\left\|p-\mathrm{L}_{\mathcal{V}}^{\text {conf }}(\mathbf{p})\right\|_{L^{2}(\Omega)} \lesssim h_{\mathrm{M}}^{2} .
$$

Proof. Let $\zeta \in H^{2}(\Omega) \cap H_{0}^{1}(\Omega)$ solve the model problem (1.1) with source $p-\mathrm{L}_{\mathcal{V}}^{\text {conf }}(\mathbf{p})$. Let $\underline{g}$ be the exact gradient, $\mathbf{g}$ the discrete gradient, and $\underline{g}^{\zeta}$ the gradient of $\zeta$. Integrating by parts yields $\left\|p-\mathrm{L}_{\mathcal{V}}^{\underline{\operatorname{conf}}}(\mathbf{p})\right\|_{L^{2}(\Omega)}^{2}=$ $\int_{\Omega}\left(\underline{g}-\underline{\operatorname{grad}}\left(\mathrm{L}_{\mathcal{V}}^{\operatorname{conf}}(\mathbf{p})\right)\right) \cdot \underline{g}^{\zeta}$ so that (recalling the approximation operator $\left.\underline{\mathrm{A}}_{\mathcal{E}}=\underline{\mathrm{L}}_{\mathcal{E}} \mathrm{R}_{\mathcal{E}}\right)$

$$
\begin{aligned}
\left\|p-\mathrm{L}_{\mathcal{V}}^{\operatorname{conf}}(\mathbf{p})\right\|_{L^{2}(\Omega)}^{2}= & \int_{\Omega}\left(\underline{g}-\underline{\mathrm{L}}_{\mathcal{E}}(\mathrm{g})\right) \cdot\left(\underline{g}^{\zeta}-\underline{\mathrm{A}}_{\mathcal{E}}\left(\underline{g}^{\zeta}\right)\right)+\int_{\Omega}\left(\underline{g}-\underline{\mathrm{L}}_{\mathcal{E}}(\mathbf{g})\right) \cdot \underline{\mathrm{A}}_{\mathcal{E}}\left(\underline{g}^{\zeta}\right) \\
& +\int_{\Omega}\left(\underline{\mathrm{L}}_{\mathcal{E}}(\mathrm{g})-\underline{\operatorname{grad}}\left(\mathrm{L}_{\mathcal{V}}^{\operatorname{conf}}(\mathbf{p})\right)\right) \cdot \underline{g}^{\zeta}:=T_{1}+T_{2}+T_{3} .
\end{aligned}
$$

The Cauchy-Schwarz inequality together with the bounds (4.14) and (4.15) yields $\left|T_{1}\right| \lesssim h_{\mathrm{M}}^{2}\|\underline{g}\|_{H^{1}(\Omega)^{3}}\left\|\underline{g}^{\zeta}\right\|_{H^{1}(\Omega)^{3}}$. Moreover, setting $\zeta=\operatorname{R}_{\mathcal{V}}(\zeta)$ and $\mathbf{g}^{\zeta}=\operatorname{GRAD}(\zeta)=\mathrm{R}_{\mathcal{E}}\left(\underline{g}^{\zeta}\right)$ so that $\underline{\mathrm{A}}_{\mathcal{E}}\left(\underline{g}^{\zeta}\right)=\underline{\mathrm{L}}_{\mathcal{E}}\left(\mathrm{g}^{\zeta}\right)$, we obtain

$$
T_{2}=\int_{\Omega} \underline{g} \cdot\left(\underline{\mathrm{L}}_{\mathcal{E}}\left(\mathrm{g}^{\zeta}\right)-\underline{\operatorname{grad}}\left(\mathrm{L}_{\mathcal{V}}^{\operatorname{conf}}(\boldsymbol{\zeta})\right)\right)+\int_{\Omega} \underline{g} \cdot \underline{\operatorname{grad}}\left(\mathrm{L}_{\mathcal{V}}^{\operatorname{conf}}(\boldsymbol{\zeta})\right)-\int_{\Omega} \underline{\mathrm{L}_{\mathcal{E}}}(\mathrm{g}) \cdot \underline{\mathrm{L}}_{\mathcal{E}}\left(\mathrm{g}^{\zeta}\right) .
$$

Using the variational form for the exact and discrete potentials and setting $A_{\mathcal{V}}^{0}:=L_{\mathcal{V}}^{0} R_{\mathcal{V}}$ while recalling that $A_{\mathcal{V}}^{\text {conf }}=L_{\mathcal{V}}^{\text {conf }} R_{\mathcal{V}}$, we infer

$$
T_{2}=\int_{\Omega} \underline{g} \cdot\left(\underline{\mathrm{L}_{\mathcal{E}}}\left(\mathrm{g}^{\zeta}\right)-\underline{\operatorname{grad}}\left(\mathrm{L}_{\mathcal{V}}^{\operatorname{conf}}(\boldsymbol{\zeta})\right)\right)+\int_{\Omega} s\left(\mathrm{~A}_{\mathcal{V}}^{\operatorname{conf}}(\zeta)-\mathrm{A}_{\mathcal{V}}^{0}(\zeta)\right):=T_{2,1}+T_{2,2} .
$$




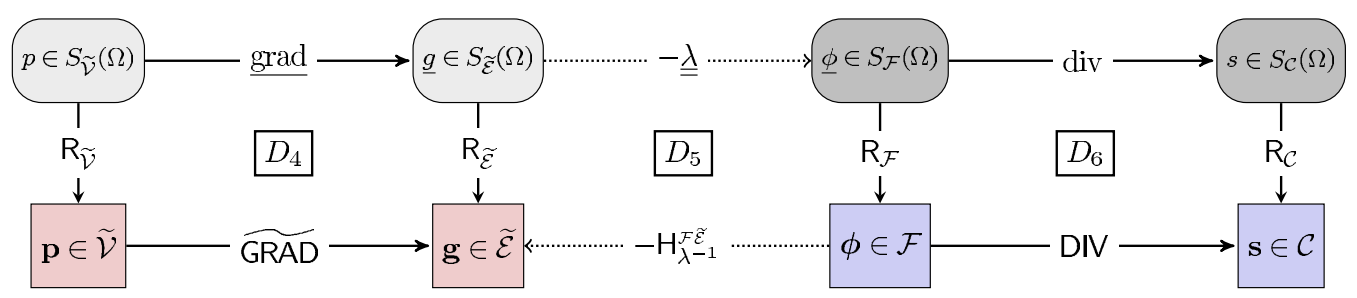

Figure 5. Tonti diagrams for the exact problem and the cell-based scheme (2.13).

To bound $T_{2,1}$, we use Lemma 4.6 (v) to subtract from $\underline{g}$ its mean-value on each primal cell yielding $\left|T_{2,1}\right| \lesssim$ $h_{\mathrm{M}}\|\underline{g}\|_{H^{1}(\Omega)^{3}}\left\|\underline{\mathbf{L}}_{\mathcal{E}}(\mathbf{g} \zeta)-\underline{\operatorname{grad}}\left(\mathrm{L}_{\mathcal{V}}^{\operatorname{conf}}(\boldsymbol{\zeta})\right)\right\|_{L^{2}(\Omega)^{3}}$, and the last factor is bounded as

$$
\begin{aligned}
\left\|\underline{\mathrm{L}}_{\mathcal{E}}\left(\mathbf{g}^{\zeta}\right)-\underline{\operatorname{grad}}\left(\mathrm{L}_{\mathcal{V}}^{\operatorname{conf}}(\boldsymbol{\zeta})\right)\right\|_{L^{2}(\Omega)^{3}} & \leq\left\|\underline{g}^{\zeta}-\underline{\mathrm{A}_{\mathcal{E}}}\left(\underline{g}^{\zeta}\right)\right\|_{L^{2}(\Omega)^{3}}+\left\|\underline{g}^{\zeta}-\underline{\operatorname{grad}}\left(\operatorname{L}_{\mathcal{V}}^{\operatorname{conf}}(\boldsymbol{\zeta})\right)\right\|_{L^{2}(\Omega)^{3}} \\
& =\left\|\underline{g}^{\zeta}-\underline{\mathrm{A}_{\mathcal{E}}}\left(\underline{g}^{\zeta}\right)\right\|_{L^{2}(\Omega)^{3}}+\left\|\underline{\operatorname{grad}}(\zeta)-\underline{\operatorname{grad}}\left(\mathrm{A}_{\mathcal{V}}^{\operatorname{conf}}(\zeta)\right)\right\|_{L^{2}(\Omega)^{3}} \lesssim h_{\mathrm{M}}\left\|\underline{g}^{\zeta}\right\|_{H^{1}(\Omega)^{3}},
\end{aligned}
$$

owing to the approximation properties of $\underline{A}_{\mathcal{E}}$ (Prop. 4.3) and $A_{\mathcal{V}}^{\text {conf }}$ (Prop. 4.7). The term $T_{2,2}$ is bounded similarly: owing to Lemma $4.6(\mathrm{v})$, the mean-value of $s$ can be subtracted from $s$ on each primal cell, so that using the regularity of $s$ yields $\left|T_{2,2}\right| \lesssim h_{\mathrm{M}}\|s\|_{H^{1}(\Omega)}\left\|\mathrm{A}_{\mathcal{V}}^{\operatorname{conf}}(\zeta)-\mathrm{A}_{\mathcal{V}}^{0}(\zeta)\right\|_{L^{2}(\Omega)}$. We add and subtract $\zeta$ in the last term on the right-hand side, use the triangle inequality together with the fact that $A_{\mathcal{V}}^{\operatorname{conf}}(\zeta)$ and $A_{\mathcal{V}}^{0}(\zeta)$ both approximate $\zeta$ to first-order in the $L^{2}$-norm to infer $\left|T_{2,2}\right| \lesssim h_{\mathrm{M}}^{2}\|s\|_{H^{1}(\Omega)}\|\zeta\|_{H^{2}(\Omega)}$. Turning to $T_{3}$, using again Lemma 4.6 (v) yields $\left|T_{3}\right| \lesssim h_{\mathrm{M}}\left\|\underline{g}^{\zeta}\right\|_{H^{1}(\Omega)^{3}}\left\|\underline{\mathrm{L}}_{\mathcal{E}}(\mathrm{g})-\underline{\operatorname{grad}}\left(\mathrm{L}_{\mathcal{V}}^{\text {conf }}(\mathbf{p})\right)\right\|_{L^{2}(\Omega)^{3}}$, and by the triangle inequality

$$
\begin{aligned}
\left\|\underline{\mathrm{L}}_{\mathcal{E}}(\mathbf{g})-\underline{\operatorname{grad}}\left(\mathrm{L}_{\mathcal{V}}^{\text {conf }}(\mathbf{p})\right)\right\|_{L^{2}(\Omega)^{3}} \leq & \left\|\underline{\mathrm{L}}_{\mathcal{E}}(\mathbf{g})-\underline{g}\right\|_{L^{2}(\Omega)^{3}}+\left\|\underline{\operatorname{grad}}(p)-\underline{\operatorname{grad}}\left(\mathrm{A}_{\mathcal{V}}^{\operatorname{conf}}(p)\right)\right\|_{L^{2}(\Omega)^{3}} \\
& +\left\|\underline{\operatorname{grad}}\left(\mathrm{L}_{\mathcal{V}}^{\operatorname{conf}}\left(\mathrm{R}_{\mathcal{V}}(p)-\mathbf{p}\right)\right)\right\|_{L^{2}(\Omega)^{3} .}
\end{aligned}
$$

The first term on the right-hand side is bounded using (4.15), the second using the approximation properties of $A_{\mathcal{V}}^{\text {conf }}$, and the third using Lemma 4.6 (iii) and Theorem 3.7. Finally, collecting the above bounds and using elliptic regularity so that $\|\zeta\|_{H^{2}(\Omega)}+\left\|\underline{g}^{\zeta}\right\|_{H^{1}(\Omega)^{3}} \lesssim\left\|p-\mathrm{L}_{\mathcal{V}}^{\text {conf }}(\mathbf{p})\right\|_{L^{2}(\Omega)}$ yields the desired $L^{2}$-error estimate.

Remark 4.9 (Conforming gradient reconstruction). It is possible to define a conforming gradient reconstruction of discrete gradients by setting $\underline{L_{\mathcal{E}}^{\text {conf }}}(\operatorname{GRAD}(\mathbf{p}))=\underline{\operatorname{grad}}\left(\mathrm{L}_{\mathcal{V}}^{\text {conf }}(\mathbf{p})\right)$ for all $\mathbf{p} \in \mathcal{V}$ (we only define this operator on the subspace $\operatorname{GRAD}(\mathcal{V})$ and not on the whole space $\mathcal{E})$. It is readily seen that $\underline{\underline{E}}_{\mathcal{E}}^{\text {conf }}: \operatorname{GRAD}(\mathcal{V}) \rightarrow H(\underline{\text { curl }} ; \Omega)$ with zero tangential component on the boundary (conformity), $\mathrm{R}_{\mathcal{E}} \underline{\underline{E}}_{\mathcal{E}}^{\text {conf }}=\mathrm{Id}_{\mathcal{E}}$ on $\operatorname{GRAD}(\mathcal{V})$ (unisolvence), $\left\|\underline{L}_{\mathcal{E}}^{\text {conf }}(\mathbf{g})\right\|_{L^{2}(\Omega)^{3}} \lesssim\|\mathbf{g}\|_{2, \mathcal{E}}$ for all $\mathbf{g}=\operatorname{GRAD}(\mathbf{p})$ (stability), $\underline{A}_{\mathcal{E}}^{\text {conf }}:=\underline{\underline{L}}_{\mathcal{E}}^{\text {conf }} \mathrm{R}_{\mathcal{E}}$ leaves invariant the gradients of piecewise affine functions on primal cells (restricted $\mathbb{P}_{0}$-consistency), and $\underline{L}_{\mathcal{E}}^{\text {conf }}(\mathrm{g})$ has the same mean-value on primal cells as $\underline{\mathrm{L}}_{\mathcal{E}}(\mathbf{g})$ for all $\mathbf{g}=\operatorname{GRAD}(\mathbf{p})$ (geometric consistency).

\section{Cell-Based schemes}

This section deals with the design and analysis of the cell-based scheme (2.13), first under an algebraic viewpoint and then using local flux reconstruction on a dual barycentric mesh. Our theoretical results are similar to those derived in Sections 3 and 4; most proofs are omitted since they hinge on an adaptation of the previous ones.

\subsection{Analysis: algebraic viewpoint}

\subsubsection{Consistency error}

For the cell-based scheme (2.13), the Tonti diagram is shown in Figure 5 . The diagrams $D_{4}$ and $D_{6}$ are commutative, but this is (in general) not the case for $D_{5}$. Since the operator $\mathrm{H}_{\lambda^{-1}-1}^{\mathcal{E}}$ is, by construction, $\mathrm{SPD}$, we 
can redefine the discrete energy and complementary energy norms such that, for all $\mathbf{g} \in \widetilde{\mathcal{E}}$ and all $\phi \in \mathcal{F}$,

$$
\|\mathbf{g}\|_{\lambda}^{2}:=\llbracket \mathbf{g},\left(\mathrm{H}_{\lambda^{\mathcal{F}}-1}\right)^{-1}(\mathbf{g}) \rrbracket_{\tilde{\mathcal{E}}_{\mathcal{F}}}, \quad\|\boldsymbol{\phi}\|_{\lambda^{-1}}^{2}:=\left[\mathrm{H}_{\lambda^{\mathcal{F}}-1}^{\tilde{\mathcal{E}}}(\boldsymbol{\phi}), \boldsymbol{\phi} \rrbracket_{\tilde{\mathcal{E}} \mathcal{F}},\right.
$$

and the basic error bound of Lemma 3.1 becomes

$$
\max \left(\left\|\mathrm{R}_{\widetilde{\mathcal{E}}}(\underline{g})-\mathrm{g}\right\|_{\lambda},\left\|\mathrm{R}_{\mathcal{F}}(\underline{\phi})-\phi\right\|_{\lambda^{-1}}\right) \leq\left\|\left\lfloor\lambda^{-1}, \mathrm{R}\right\rceil(\underline{\phi})\right\|_{\lambda},
$$

where $\left\lfloor\lambda^{-1}, \mathrm{R}\right\rceil:=\mathrm{H}_{\lambda^{-}-1}^{\mathcal{F}} \mathrm{R}_{\mathcal{F}}-\mathrm{R}_{\tilde{\mathcal{E}}} \underline{\underline{\lambda}}^{-1}$ is the commuting operator for diagram $D_{5}$ of Figure 5 .

\subsubsection{Local discrete Hodge operator}

The discrete Hodge operator $\mathrm{H}_{\lambda^{-1}}^{\mathcal{F}}$ is built by assembling local discrete Hodge operators attached to primal cells. Let $c \in \mathrm{C}$ and consider the sets $\mathrm{F}_{c}:=\{f \in \mathrm{F} ; f \subset \partial c\}$, collecting the primal faces of $c$, and $\widetilde{\mathrm{E}}_{c}:=$ $\left\{\tilde{e}_{c}(f):=\tilde{e}(f) \cap c ; f \in \mathrm{F}_{c}\right\}$, collecting the dual subedges of $c$. The local de Rham maps $\mathrm{R}_{\widetilde{\mathcal{E}}_{c}}: S_{\widetilde{\mathcal{E}}}(c) \rightarrow \widetilde{\mathcal{E}}_{c}$ and $\mathrm{R}_{\mathcal{F}_{c}}: S_{\mathcal{F}}(c) \rightarrow \mathcal{F}_{c}$ are such that, for all $f \in \mathrm{F}_{c},\left(\mathrm{R}_{\widetilde{\mathcal{E}}_{c}}(\underline{g})\right)_{\tilde{e}_{c}(f)}:=\int_{\tilde{e}_{c}(f)} \underline{g} \cdot \underline{\tau}_{\tilde{e}_{c}(f)}$ and $\left(\mathrm{R}_{\mathcal{F}_{c}}(\underline{\phi})\right)_{f}:=\int_{f} \underline{\phi} \cdot \underline{\nu}_{f}$ where $\underline{\tau}_{\tilde{e}_{c}(f)}$ is consistently oriented by the fixed unit normal $\underline{\nu}_{f}$ to $f$. The assembly of the operator $\mathrm{H}_{\lambda^{\mathcal{F}}-1}$ takes the form $\mathrm{H}_{\lambda-1}^{\mathcal{F} \tilde{\mathcal{E}}}=\sum_{c \in \mathrm{C}} \mathrm{P}_{\mathcal{F}, c}^{*} \mathrm{H}_{\lambda-1}^{\mathcal{F}_{\mathcal{c}} \tilde{\mathcal{E}}_{c}} \mathrm{P}_{\mathcal{F}, c}$, with local discrete Hodge operators $\mathrm{H}_{\lambda-1}^{\mathcal{F}_{c} \tilde{\mathcal{E}}_{c}}: \mathcal{F}_{c} \rightarrow \widetilde{\mathcal{E}}_{c}$, while $\mathrm{P}_{\mathcal{F}, c}: \mathcal{F} \rightarrow \mathcal{F}_{c}$ is the (full-rank) map from global to local degrees of freedom on faces and $\mathrm{P}_{\mathcal{F}, c}^{*}$ its adjoint. The two properties to be satisfied by the local discrete Hodge operators $\mathrm{H}_{\lambda^{-1}}^{\mathcal{F}_{c} \tilde{\mathcal{c}}_{c}}$ are the following: for all $c \in \mathrm{C}$,

(A1c) [Stability] $\mathrm{H}_{\lambda-1}^{\mathcal{F}_{\mathcal{c}} \tilde{\mathcal{E}}_{c}}$ is symmetric positive definite, and there is $\eta>0$ such that, for all $\phi \in \mathcal{F}_{c}$,

$$
\eta \lambda_{\sharp, c}^{-1}\|\phi\|_{2, \mathcal{F}_{c}}^{2} \leq \llbracket \mathrm{H}_{\lambda^{-1}}^{\mathcal{F}_{c} \tilde{\mathcal{E}}_{c}}(\boldsymbol{\phi}), \phi \rrbracket_{\tilde{\mathcal{E}}_{c} \mathcal{F}_{c}} \leq \eta^{-1} \lambda_{b, c}^{-1}\|\phi\|_{2, \mathcal{F}_{c}}^{2}, \quad\|\phi\|_{2, \mathcal{F}_{c}}^{2}:=\sum_{f \in \mathrm{F}_{c}}\left|f \| \tilde{e}_{c}(f)\right|\left(\frac{\phi_{f}}{|f|}\right)^{2} .
$$

(A2c) $\left[\mathbb{P}_{0}\right.$-consistency] Introducing the local commuting operator $\left\lfloor\lambda^{-1}, \mathrm{R}\right]_{c}:=\mathrm{H}_{\lambda^{-1}}^{\mathcal{F}_{c} \tilde{\mathcal{E}}_{c}} \mathrm{R}_{\mathcal{F}_{c}}-\mathrm{R}_{\widetilde{\mathcal{E}}_{c}} \underline{\underline{\lambda}}^{-1}$, there holds, for all $\underline{\Phi}$ constant in $c,\left\lfloor\lambda^{-1}, \mathrm{R}\right\rceil_{c}(\underline{\Phi})=0$.

The tighter error bound of Lemma 3.2 becomes

$$
\max \left(\left\|\mathrm{R}_{\widetilde{\mathcal{E}}}(\underline{g})-\mathbf{g}\right\|\left\|_{\lambda},\right\| \mathrm{R}_{\mathcal{F}}(\underline{\phi})-\phi \|_{\lambda^{-1}}\right) \leq \inf _{\underline{\Phi} \in\left[\mathbb{P}_{0}(\mathrm{C})\right]^{3}}\left\{\sum_{c \in \mathrm{C}}\left\|\left[\lambda^{-1}, \mathrm{R}\right]_{c}\left(\left.(\underline{\phi}-\underline{\Phi})\right|_{c}\right)\right\|_{\lambda, c}^{2}\right\}^{1 / 2} .
$$

As for the vertex-based scheme, if the exact solution turns out to be piecewise affine on the primal mesh, the solution of the cell-based scheme is the image by the de Rham map of the exact solution.

\subsubsection{Discrete functional analysis and discrete stability}

To allow for more generality, we consider in this section an arbitrary dimension $d>1$. For a real number $1 \leq p<+\infty$, we define the following discrete norms: for all $\mathbf{p} \in \widetilde{\mathcal{V}}$ and all $\mathbf{g} \in \widetilde{\mathcal{E}}$,

$$
\|\mathbf{p}\|_{p, \tilde{\mathcal{V}}}^{p}:=\sum_{c \in \mathrm{C}}|c|\left|\mathbf{p}_{\tilde{v}(c)}\right|^{p}, \quad\|\mathbf{g}\|_{p, \tilde{\mathcal{E}}}^{p}:=\sum_{c \in \mathrm{C}} \sum_{f \in \mathrm{F}_{c}}|f|\left|\tilde{e}_{c}(f)\right|\left(\frac{\left|\mathbf{g}_{\tilde{e}(f)}\right|}{|\tilde{e}(f)|}\right)^{p} .
$$

For all $\mathbf{p} \in \widetilde{\mathcal{V}}, \mathrm{L}_{\tilde{\mathcal{V}}}^{0}(\mathbf{p})$ is piecewise constant on the primal mesh with $\left.\mathrm{L}_{\tilde{\mathcal{V}}}^{0}(\mathbf{p})\right|_{c}=\mathbf{p}_{\tilde{v}(c)}$, and extended by zero outside $\Omega$. Then, similarly to Theorem 3.4, there holds $\left\|\mathrm{L}_{\widetilde{\mathcal{V}}}^{0}(\mathbf{p})\right\|_{\mathrm{BV}} \leq \sqrt{d}\|\widetilde{\mathrm{GRAD}}(\mathbf{p})\|_{1, \widetilde{\mathcal{E}}}$. The counterpart of Theorem 3.5 hinges, similarly to (3.13), on the existence of $\tilde{\gamma}>0$ such that, for all $\tilde{v} \in \widetilde{\mathrm{V}}$,

$$
\tilde{\gamma} \sum_{\tilde{e} \in \widetilde{\mathrm{E}}_{\tilde{v}}}|\tilde{e}||f(\tilde{e})| \leq|c(\tilde{v})| \text {. }
$$

Recall that the discrete operator $\widetilde{\text { GRAD }}$ explicitly accounts for the homogeneous Dirichlet boundary condition on dual edges touching the boundary. 
Theorem 5.1 (Discrete Sobolev embedding). Let $d>1$. Let $1 \leq p<+\infty$. Assume (5.6). Then, for all $q$ as in Theorem 3.5, there is $\tilde{C}_{p, q}$ such that, for all $\mathbf{p} \in \tilde{\mathcal{V}},\|\mathbf{p}\|_{q, \tilde{\mathcal{V}}} \leq \tilde{C}_{p, q}\|\widetilde{\operatorname{GRAD}}(\mathbf{p})\|_{p, \tilde{\mathcal{E}}}$. In particular, the choice $p=q=2$ leads to the discrete Poincaré inequality

$$
\|\mathbf{p}\|_{2, \tilde{\mathcal{V}}} \leq \tilde{C}_{2,2}\|\widetilde{\operatorname{GRAD}}(\mathbf{p})\|_{2, \widetilde{\mathcal{E}}}
$$

A simple consequence of the discrete Poincaré inequality is the stability of the cell-based scheme. Indeed, let $(\mathbf{p}, \phi) \in \widetilde{\mathcal{V}} \times \mathcal{F}$ solve $(2.13)$. Then, since $\|\widetilde{\operatorname{GRAD}}(\mathbf{p})\|_{2, \widetilde{\mathcal{E}}} \lesssim\left(\eta \lambda_{b}\right)^{-1 / 2}\|\phi\|_{\lambda^{-1}}$, there holds

$$
\|\phi\|_{\lambda^{-1}} \leq\left(\eta \lambda_{b}\right)^{-1 / 2} \tilde{C}_{2,2}\|s\|_{L^{2}(\Omega)}, \quad\|\mathbf{p}\|_{2, \tilde{\mathcal{V}}} \leq \eta \lambda_{b}\left(\tilde{C}_{2,2}\right)^{2}\|s\|_{L^{2}(\Omega)} .
$$

\subsubsection{Convergence rate for smooth solutions}

Theorem 5.2 (Convergence rate for smooth solutions). Let $\underline{g}$ be the exact gradient and $\phi$ the exact flux. Let $\mathrm{g}$ be the discrete gradient and $\phi$ the discrete flux resulting from (2.13). Assume $\underline{g}, \underline{\phi} \in\left[\bar{H}^{1}(\mathrm{C})\right]^{3}$. Assume the meshes satisfy (M1). Let $h_{\mathrm{M}}$ denote the maximal diameter of primal cells. Then,

$$
\max \left(\left\|\mathrm{R}_{\tilde{\mathcal{E}}}(\underline{g})-\mathbf{g}\right\|_{\lambda},\left\|\mathrm{R}_{\mathcal{F}}(\underline{\phi})-\phi\right\|_{\lambda^{-1}}\right) \lesssim h_{\mathrm{M}}\left(\lambda_{\sharp}^{1 / 2}\|\underline{g}\|_{H^{1}(\mathrm{C})^{3}}+\lambda_{\mathrm{b}}^{-1 / 2}\|\underline{\phi}\|_{H^{1}(\mathrm{C})^{3}}\right) .
$$

\subsection{Analysis based on flux reconstruction}

As in Section 4.1, we assume planar primal faces that are star-shaped with respect to their barycenter and consider a dual barycentric mesh. Letting $\underline{f}:=\int_{f} \underline{\nu}_{f}$ and $\underline{\tilde{e}}_{c}(f):=\int_{\tilde{e}_{c}(f)} \underline{\tau}_{\tilde{e}(f)}$, the counterpart of (4.1) is $\sum_{f \in \mathrm{F}_{c}} \underline{\tilde{e}}_{c}(f) \otimes \underline{f}=\sum_{f \in \mathrm{F}_{c}} \underline{f} \otimes \underline{\tilde{e}}_{c}(f)=|c| \underline{\underline{\mathrm{d}}}$.

\subsubsection{Local flux reconstruction and local discrete Hodge operator}

Let $c \in \mathrm{C}$. We consider a family of $\# \mathrm{~F}_{c}$ linearly independent functions $\underline{\ell}_{f, c}: c \rightarrow \mathbb{R}^{3}$ spanning a finitedimensional space of vector-valued functions denoted $P_{\mathcal{F}}(c) \subset\left[L^{2}(c)\right]^{3}$. We introduce the local lifting operator

$$
\underline{\mathrm{L}}_{\mathcal{F}_{c}}: \mathcal{F}_{c} \ni \phi \longmapsto \underline{\mathrm{L}}_{\mathcal{F}_{c}}(\phi):=\sum_{f \in \mathrm{F}_{c}} \phi_{f} \underline{\ell}_{f, c}(\underline{x}) \in P_{\mathcal{F}}(c)
$$

Functions in $P_{\mathcal{F}}(c)$ are assumed to be sufficiently smooth so that the local reduction operator $\mathrm{R}_{\mathcal{F}_{c}}$ can act on them. Typically, functions in $P_{\mathcal{F}}(c)$ are piecewise polynomials on a submesh of $c$ composed of elementary subsimplices or aggregations thereof; we stress that the normal component of these functions is not necessarily continuous across the faces of this submesh lying inside $c$, so that, in general, $P_{\mathcal{F}}(c) \not \subset H$ (div; $c$ ). The requirements on the local reconstruction functions $\left\{\underline{\ell}_{f, c}\right\}_{f \in \mathrm{F}_{c}}$ are:

(R1c) [Unisolvence]. For all $f, f^{\prime} \in \mathrm{F}_{c}, \int_{f^{\prime}} \underline{\ell}_{f, c}(\underline{x}) \cdot \underline{\nu}_{f^{\prime}}=\delta_{f, f^{\prime}}$.

(R2c) [Stability]. There is $\eta>0$ such that, for all $\phi \in \mathcal{F}_{c}, \eta\|\phi\|_{2, \mathcal{F}_{c}}^{2} \leq\left\|\underline{\mathrm{L}}_{\mathcal{F}_{c}}(\boldsymbol{\phi})\right\|_{L^{2}(c)^{3}}^{2} \leq \eta^{-1}\|\phi\|_{2, \mathcal{F}_{c}}^{2}$.

(R3c) [Partition of unity]. For all $\underline{x} \in c, \sum_{f \in \mathrm{F}_{c}} \underline{\ell}_{f, c}(\underline{x}) \otimes \underline{f}=\underline{\underline{\mathrm{Id}}}$.

(R4c) [Geometric consistency]. For all $f \in \mathrm{F}_{c}$, there holds $\int_{c} \underline{\ell}_{f, c}(\underline{x})=\underline{\tilde{e}}_{c}(f)$.

As shown by Codecasa et al. [13,14], (R1c) is equivalent to $\mathbf{R}_{\mathcal{F}_{c}} \underline{\underline{F}}_{\mathcal{F}_{c}}=\mathrm{Id}_{\mathcal{F}_{c}}$, and (R3c) to the fact that, for all $\underline{\Phi}$ constant in $c, \underline{\mathrm{L}}_{\mathcal{F}_{c}} \mathrm{R}_{\mathcal{F}_{c}}(\underline{\Phi})=\underline{\Phi}$.

Definition 5.3. The local discrete Hodge operator is defined by specifying its entries such that, for all $f, f^{\prime} \in \mathrm{F}_{c}$,

$$
\left(\mathrm{H}_{\lambda^{-1}}^{\mathcal{F}_{c} \tilde{\varepsilon}_{c}}\right)_{f, f^{\prime}}:=\int_{c} \underline{\underline{\lambda}}^{-1} \underline{\ell}_{f, c}(\underline{x}) \cdot \underline{\ell}_{f^{\prime}, c}(\underline{x})
$$



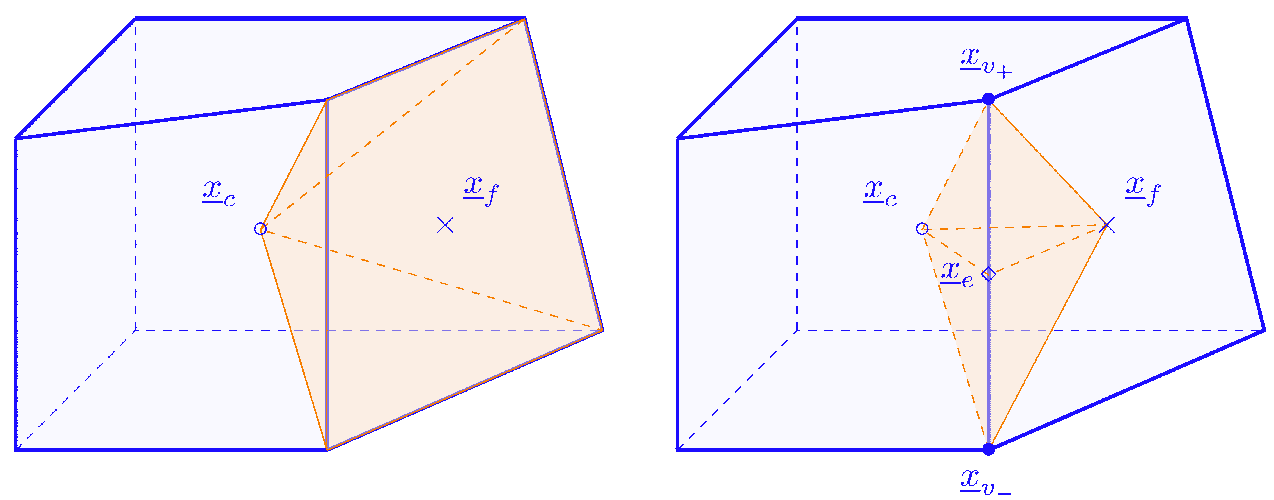

Figure 6. Left: elementary subvolume considered for the flux reconstruction in the Discrete Geometric Approach; Right: elementary subvolume considered for the conforming potential reconstruction in Section B.1.

Proposition 5.4. Let $\mathrm{H}_{\lambda^{-1}}^{\mathcal{F}_{c} \tilde{\mathcal{E}}_{c}}$ be defined by (5.11). Assume (R1c)-(R4c). Then, (A1c) and (A2c) hold.

\subsubsection{Examples}

On Cartesian meshes, one possibility is to set, for all $c \in \mathrm{C}$ (with fully barycentric subdivision) and all $f \in \mathrm{F}_{c}, \underline{\ell}_{f, c}^{\mathrm{CAR}}(x):=|f|^{-1} \underline{\nu}_{f}$ on $\mathfrak{p}_{f, c}^{\mathrm{CAR}}:=\cup_{v \in \mathrm{V}_{f}} \cup_{e \in \mathrm{E}_{v} \cap \mathrm{E}_{c}} \cup_{f^{\prime} \in \mathrm{F}_{e} \cap \mathrm{F}_{c}} \mathfrak{s}\left(\underline{x}_{v}, \underline{x}_{e}, \underline{x}_{f^{\prime}}, \underline{x}_{c}\right)$ and $\underline{\ell}_{f, c}^{\mathrm{CAR}}(x):=\underline{0}$ otherwise. Then, properties (R1c)-(R4c) are easily verified. Moreover, for isotropic conductivity, the local discrete Hodge operator defined by (5.11) is diagonal and corresponds to (2.15).

On tetrahedral meshes, one possibility is to set, for all $c \in \mathrm{C}$ and all $f \in \mathrm{F}_{c}, \underline{\ell}_{f, c}^{\mathrm{WHI}}(\underline{x}):=\left.\underline{\varphi}_{f}^{\mathrm{RTN}}\right|_{c}(\underline{x})$, where $\underline{\varphi}_{f}^{\mathrm{RTN}}$ is the lowest-order Raviart-Thomas-Nédélec finite element function attached to $f$. Properties (R1c)-(R4c) are easily verified. The cell-based scheme (2.13) coincides with the classical lowest-order mixed finite element approximation on the primal mesh.

On polyhedral meshes, one possibility is to use the Discrete Geometric Approach of Codecasa et al. $[13,14]$.

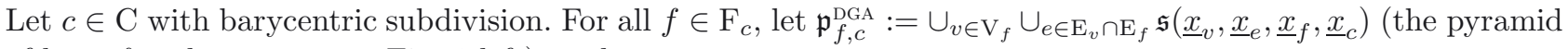
of base $f$ and apex $\underline{x}_{c}$, see Fig. 6 left), and set

$$
\forall f^{\prime} \in \mathrm{F}_{c},\left.\quad \underline{\ell}_{f, c}^{\mathrm{DGA}}\right|_{\mathfrak{p}_{f^{\prime}, c}^{\mathrm{DGA}}}:=\frac{\underline{\tilde{e}}_{c}(f)}{\underline{\tilde{e}}_{c}(f) \cdot \underline{f}} \delta_{f, f^{\prime}}+\left(\underline{\underline{\mathrm{Id}}}-\frac{\underline{\tilde{e}}_{c}\left(f^{\prime}\right) \otimes \underline{f^{\prime}}}{\underline{\tilde{e}}_{c}\left(f^{\prime}\right) \cdot \underline{f^{\prime}}}\right) \frac{\underline{\tilde{e}}_{c}(f)}{|c|},
$$

so that $\underline{\ell}_{f, c}^{\mathrm{DGA}}$ is piecewise constant on the submesh $\left\{\mathfrak{p}_{f^{\prime}, c}^{\mathrm{DGA}}\right\}_{f^{\prime} \in \mathrm{F}_{c}}$ of $c$. Properties (R1c), (R3c), and (R4c) are shown in [15], while property $(\mathbf{R} 2 \mathbf{c})$ is readily verified using mesh regularity.

\subsubsection{Global flux reconstruction and discrete Hodge operator}

The global flux reconstruction operator $\underline{\underline{L}}_{\mathcal{F}}: \mathcal{F} \rightarrow P_{\mathcal{F}}(\mathrm{C}) \subset\left[L^{2}(\Omega)\right]^{3}$ is defined such that, for all $\phi \in \mathcal{F}$, $\left.\underline{\mathrm{L}}_{\mathcal{F}}(\phi)\right|_{c}:=\underline{\mathrm{L}}_{\mathcal{F}_{c}}\left(\mathrm{P}_{\mathcal{F}, c}(\phi)\right)$ for all $c \in \mathrm{C}$. This operator maps onto the broken polynomial space $P_{\mathcal{F}}(\mathrm{C}):=\{\underline{\phi} \in$ $\left.\left[L^{2}(c)\right]^{3} ; \forall c \in \mathrm{C},\left.\phi\right|_{c} \in P_{\mathcal{F}}(c)\right\}$ which is, in general, nonconforming in $H(\operatorname{div} ; \Omega)$. Owing to (R1c), the normal component of $\underline{L}_{\mathcal{F}}(\bar{\phi})$ is single-valued across all primal faces $f \in \mathrm{F}$, so that $\mathrm{R}_{\mathcal{F}}$ can act on $\underline{\mathrm{L}}_{\mathcal{F}}(\phi)$, and there holds $\mathrm{R}_{\mathcal{F}} \underline{\mathrm{F}}_{\mathcal{F}}=\mathrm{Id}_{\mathcal{F}}$. The discrete Hodge operator is such that

$$
\forall \phi_{1}, \phi_{2} \in \mathcal{F}, \quad \llbracket \mathrm{H}_{\lambda}^{\mathcal{F} \tilde{\mathcal{E}}}\left(\phi_{1}\right), \phi_{2} \rrbracket_{\tilde{\mathcal{E}} \mathcal{F}}=\int_{\Omega} \underline{\underline{\lambda}}^{-1} \underline{\mathrm{L}}_{\mathcal{F}}\left(\phi_{1}\right) \cdot \underline{\mathrm{L}}_{\mathcal{F}}\left(\phi_{2}\right)
$$

The nonconforming approximation map $\underline{\mathrm{A}}_{\mathcal{F}}:=\underline{\mathrm{L}}_{\mathcal{F}} \mathrm{R}_{\mathcal{F}}: S_{\mathcal{F}}(\Omega) \rightarrow P_{\mathcal{F}}(\mathrm{C})$ is a projector, it satisfies, for all piecewise constant $\underline{\Phi}$ on the primal mesh, $\underline{\mathrm{A}}_{\mathcal{F}}(\underline{\Phi})=\underline{\Phi}$, and, for all $\underline{\phi} \in\left[H^{1}(\mathrm{C})\right]^{3} \cap S_{\mathcal{F}}(\Omega)$, assuming that the 
barycentric subdivision satisfies (M1), there holds

$$
\left\|\underline{\phi}-\underline{\mathrm{A}}_{\mathcal{F}}(\underline{\phi})\right\|_{L^{2}(\Omega)^{3}} \lesssim h_{\mathrm{M}}\|\underline{\phi}\|_{H^{1}(\mathrm{C})^{3}} .
$$

Corollary 5.5 (Error estimate on reconstructed gradient and flux). Let g be the exact gradient and $\phi$ the exact flux. Let $\mathbf{g}$ be the discrete gradient and $\boldsymbol{\phi}$ the discrete flux resulting from $(2.13)$. Assume $\underline{g}, \underline{\phi} \in\left[H^{1}(\mathrm{C})\right]^{3}$. Assume that the barycentric subdivision satisfies (M1). Then, letting $\underline{\underline{L}}_{\tilde{\mathcal{E}}}(\mathrm{g}):=-\underline{\underline{\lambda}}^{-1} \underline{\underline{L}}_{\mathcal{F}}(\phi)$,

$$
\left\|\underline{g}-\underline{\mathrm{L}}_{\tilde{\mathcal{E}}}(\mathrm{g})\right\|_{\lambda}=\left\|\underline{\phi}-\underline{\mathrm{L}}_{\mathcal{F}}(\phi)\right\|_{\lambda^{-1}} \lesssim h_{\mathrm{M}}\left(\lambda_{\sharp}^{1 / 2}\|\underline{g}\|_{H^{1}(\mathrm{C})^{3}}+\lambda_{\mathrm{b}}^{-1 / 2}\|\underline{\phi}\|_{H^{1}(\mathrm{C})^{3}}\right) .
$$

\subsection{4. $L^{2}$-error estimate}

For simplicity, we assume in this section that $\underline{\underline{\lambda}}$ is the identity tensor. Moreover, we assume a fully barycentric subdivision. The proof of our $L^{2}$-error estimate proceeds somewhat differently than that for the vertex-based scheme; herein, we consider a conforming flux reconstruction. Let $\mathrm{L}_{\mathcal{C}}: \mathcal{C} \rightarrow L^{2}(\Omega)$ reconstruct piecewise constant functions on the primal mesh with $\left.\mathrm{L}_{\mathcal{C}}(\mathbf{s})\right|_{\mathcal{c}}=|c|^{-1} \mathbf{s}_{\mathcal{c}}$ for all $c \in \mathrm{C}$ and for all $\mathbf{s} \in \mathcal{C}$; clearly $\mathrm{R}_{\mathcal{C}} \mathrm{L}_{\mathcal{C}}=\operatorname{ld}_{\mathcal{C}}$.

Lemma 5.6. Assume a fully barycentric subdivision satisfying (M1) and planar primal faces. Then, there exists a conforming flux reconstruction operator $\underline{\underline{L}}_{\mathcal{F}}^{\text {conf }}$ built from local operators $\underline{L}_{\mathcal{F}}^{\text {conf }}$ by setting $\left.\underline{L}_{\mathcal{F}}^{\text {conf }}\right|_{c}:=\underline{L}_{\mathcal{F}_{c}}^{\text {conf }}$ for all $c \in \mathrm{C}$ and satisfying the following properties:

(i) (Conformity) $\underline{\underline{L}}_{\mathcal{F}}^{\text {conf }}: \mathcal{F} \rightarrow H(\operatorname{div} ; \Omega), \operatorname{div}\left(\underline{\operatorname{L}}_{\mathcal{F}}^{\text {conf }}(\phi)\right)=\operatorname{L}_{\mathcal{C}}(\operatorname{DIV}(\phi))$ for all $\phi \in \mathcal{F}$;

(ii) (Unisolvence) $\mathrm{R}_{\mathcal{F}} \underline{\underline{F}}_{\mathcal{F}}^{\text {conf }}=\mathrm{Id}_{\mathcal{F}}$;

(iii) (Stability) For all $c \in \mathrm{C}$ and all $\phi \in \mathcal{F}_{c},\left\|\underline{\mathcal{L}}_{c}^{\operatorname{conf}}(\phi)\right\|_{L^{2}(c)^{3}} \lesssim\|\phi\|_{2, \mathcal{F}_{c}}$;

(iv) $\left(\mathbb{P}_{0}\right.$-consistency) For all $c \in \mathrm{C}$, the approximation map $\underline{\mathrm{A}}_{\mathcal{F}_{c}}^{\text {conf }}:=\underline{\mathrm{L}}_{\mathcal{F}_{c}}^{\text {conf }} \mathrm{R}_{\mathcal{F}_{c}}$ preserves piecewise constant vector fields, so that, setting $\underline{\mathrm{A}}_{\mathcal{F}}^{\text {conf }}:=\underline{\mathrm{L}}_{\mathcal{F}}^{\text {conf }} \mathrm{R}_{\mathcal{F}}$, there holds $\left\|\underline{\phi}-\underline{\mathrm{A}}_{\mathcal{F}}^{\text {conf }}(\underline{\phi})\right\|_{L^{2}(\Omega)^{3}} \lesssim h_{\mathrm{M}}\|\underline{\phi}\|_{H^{1}(\Omega)^{3}}$ for all $\underline{\phi} \in\left[H^{1}(\Omega)\right]^{3} \cap S_{\mathcal{F}}(\Omega) ;$

(v) (Geometric consistency) For all $\phi \in \mathcal{F}, \underline{L}_{\mathcal{F}}(\phi)$ and $\underline{L}_{\mathcal{F}}^{\text {conf }}(\phi)$ have the same mean-value on primal cells.

The proof is sketched in Appendix B. We now turn to the main result of this section; note that the $L^{2}$-error estimate is established with respect to the mean-value of the exact potential on primal cells, as is classical in mixed finite element and cell-centered finite volume schemes.

Theorem 5.7. Assume that the model problem (1.1) with $\underline{\underline{\lambda}}=\underline{\underline{\mathrm{Id}}}$ has elliptic regularity. Assume $s \in H^{1}(\Omega)$. Let $p$ be the exact potential and let $\mathbf{p}$ be the discrete potential resulting from (2.13). Assume a fully barycentric subdivision satisfying (M1) and planar primal faces. Recall $\mathrm{L}_{\tilde{\mathcal{V}}}^{0}$ from Section 5.1.3. Then,

$$
\left\|\mathrm{L}_{\mathcal{C}} \mathrm{R}_{\mathcal{C}}(p)-\mathrm{L}_{\tilde{\mathcal{V}}}^{0}(\mathbf{p})\right\|_{L^{2}(\Omega)} \lesssim h_{\mathrm{M}}^{2} .
$$

Proof. Let $\zeta \in H^{2}(\Omega) \cap H_{0}^{1}(\Omega)$ solve the model problem (1.1) with source $\mathrm{L}_{\mathcal{C}} \mathrm{R}_{\mathcal{C}}(p)-\mathrm{L}_{\tilde{\mathcal{V}}}^{0}(\mathbf{p})$. Let $\underline{\phi}$ be the exact flux, $\phi$ the discrete flux, and $\underline{\phi}^{\zeta}$ the flux of $\zeta$. Observing that $\left.\mathrm{L}_{\mathcal{C}} \mathrm{R}_{\mathcal{C}}(p)\right|_{c}=|c|^{-1} \int_{c} p$, we infer

$$
\left\|\mathrm{L}_{\mathcal{C}} \mathrm{R}_{\mathcal{C}}(p)-\mathrm{L}_{\tilde{\mathcal{V}}}^{0}(\mathbf{p})\right\|_{L^{2}(\Omega)}^{2}=\int_{\Omega}\left(p-\mathrm{L}_{\tilde{\mathcal{V}}}^{0}(\mathbf{p})\right) \mathrm{L}_{\mathcal{C}}\left(\mathrm{R}_{\mathcal{C}}\left(\operatorname{div} \underline{\phi}^{\zeta}\right)\right)=\int_{\Omega} \underline{\phi} \cdot \underline{\mathrm{A}}_{\mathcal{F}}^{\operatorname{conf}}\left(\underline{\phi}^{\zeta}\right)-\int_{\Omega} \mathrm{L}_{\mathcal{F}}(\phi) \cdot \underline{\mathrm{A}}_{\mathcal{F}}\left(\underline{\phi}^{\zeta}\right)
$$

since $\mathrm{L}_{\mathcal{C}}\left(\mathrm{R}_{\mathcal{C}}\left(\operatorname{div} \underline{\phi}^{\zeta}\right)\right)=\mathrm{L}_{\mathcal{C}}\left(\operatorname{DIV}\left(\mathrm{R}_{\mathcal{F}}\left(\underline{\phi}^{\zeta}\right)\right)\right)=\operatorname{div}\left(\operatorname{L}_{\mathcal{F}}^{\operatorname{conf}}\left(\mathrm{R}_{\mathcal{F}}\left(\underline{\phi}^{\zeta}\right)\right)\right)=\operatorname{div}\left(\underline{A}_{\mathcal{F}}^{\operatorname{conf}}\left(\underline{\phi}^{\zeta}\right)\right)$ so that $\int_{\Omega} p \mathrm{~L}_{\mathcal{C}}\left(\mathrm{R}_{\mathcal{C}}\left(\operatorname{div} \underline{\phi}^{\zeta}\right)\right)=$ $\int_{\Omega} \underline{\phi} \cdot \underline{A}_{\mathcal{F}}^{\text {conf }}\left(\underline{\phi}^{\zeta}\right)$ using integration by parts, and since, using the adjunction of GRAD and DIV, the fact that $\widehat{\operatorname{GRAD}}(\mathbf{p})=-\mathrm{H}_{\lambda^{-1}}^{\mathcal{F}}(\phi)$, and the definition (5.13) of $\mathrm{H}_{\lambda^{-1}}^{\mathcal{F}}$,

$$
\int_{\Omega} \mathrm{L}_{\tilde{\mathcal{V}}}^{0}(\mathbf{p}) \mathrm{L}_{\mathcal{C}}\left(\mathrm{R}_{\mathcal{C}}\left(\operatorname{div} \underline{\phi}^{\zeta}\right)\right)=\llbracket \mathbf{p}, \mathrm{R}_{\mathcal{C}}\left(\operatorname{div} \underline{\phi}^{\zeta}\right) \rrbracket_{\tilde{\mathcal{V}} \mathcal{C}}=\llbracket \mathbf{p}, \operatorname{DIV}\left(\mathrm{R}_{\mathcal{F}}\left(\underline{\phi}^{\zeta}\right)\right) \rrbracket_{\tilde{\mathcal{V}} \mathcal{C}}=\int_{\Omega} \mathrm{L}_{\mathcal{F}}(\phi) \cdot \underline{\mathrm{A}}_{\mathcal{F}}\left(\underline{\phi}^{\zeta}\right) .
$$




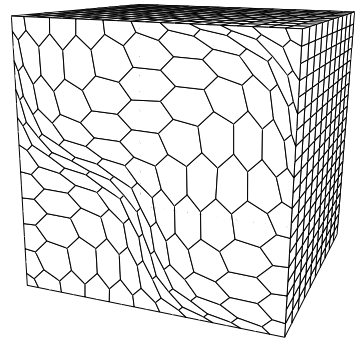

(A) $\mathrm{n}_{\mathrm{sys}}=1800$

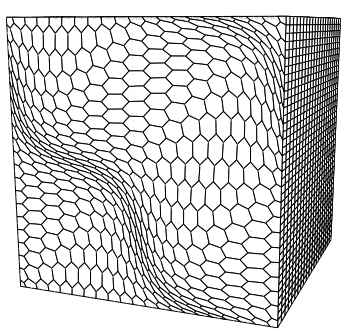

(в) $\mathrm{n}_{\mathrm{sys}}=15200$

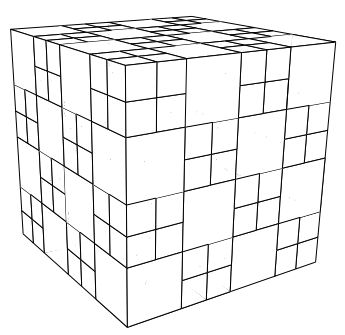

(c) $\mathrm{n}_{\mathrm{sys}}=311$

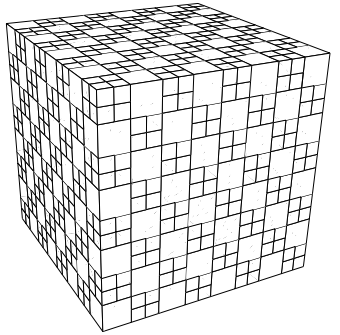

(D) $\mathrm{n}_{\mathrm{sys}}=3119$

Figure 7. Two examples of prismatic meshes with polygonal basis (left) and of checkerboard meshes (right).

As a result,

$$
\begin{aligned}
\left\|\mathrm{L}_{\mathcal{C}} \mathrm{R}_{\mathcal{C}}(p)-\mathrm{L}_{\tilde{\mathcal{V}}}^{0}(\mathbf{p})\right\|_{L^{2}(\Omega)}^{2}= & -\int_{\Omega}\left(\underline{\phi}-\underline{\mathrm{L}}_{\mathcal{F}}(\phi)\right) \cdot\left(\underline{\phi}^{\zeta}-\underline{\mathrm{A}}_{\mathcal{F}}\left(\underline{\phi}^{\zeta}\right)\right)+\int_{\Omega}\left(\underline{\phi}-\underline{\mathrm{L}}_{\mathcal{F}}^{\operatorname{conf}}(\phi)\right) \cdot \underline{\phi}^{\zeta} \\
& +\int_{\Omega}\left(\underline{\mathrm{L}}_{\mathcal{F}}^{\operatorname{conf}}-\underline{\mathrm{L}}_{\mathcal{F}}\right)(\phi) \cdot \underline{\phi}^{\zeta}+\int_{\Omega} \underline{\phi} \cdot\left(\underline{\mathrm{A}}_{\mathcal{F}}^{\operatorname{conf}}-\underline{\mathrm{A}}_{\mathcal{F}}\right)\left(\underline{\phi}^{\zeta}\right):=T_{1}+T_{2}+T_{3}+T_{4} .
\end{aligned}
$$

$T_{1}$ is bounded using (5.15) and (5.14). Using integration by parts, Lemma 5.6 (i) and the cell-based scheme (2.13),

$T_{2}=\int_{\Omega} \operatorname{div}\left(\underline{\phi}-\underline{\mathrm{L}}_{\mathcal{F}}^{\operatorname{conf}}(\phi)\right) \zeta=\int_{\Omega}\left(\operatorname{div} \underline{\phi}-\mathrm{L}_{\mathcal{C}}(\operatorname{DIV}(\phi))\right) \zeta=\int_{\Omega}\left(s-\mathrm{L}_{\mathcal{C}} \mathrm{R}_{\mathcal{C}}(s)\right) \zeta=\int_{\Omega}\left(s-\mathrm{L}_{\mathcal{C}} \mathrm{R}_{\mathcal{C}}(s)\right)\left(\zeta-\mathrm{L}_{\tilde{\mathcal{V}}}^{0} \mathrm{R}_{\widetilde{\mathcal{V}}}(\zeta)\right)$,

which is easily bounded since $s \in H^{1}(\Omega)$. Finally, $T_{3}$ and $T_{4}$ are bounded as in the proof of Theorem 4.8.

\section{Numerical Results}

We investigate numerically the vertex-based scheme (2.9). The discrete Hodge operator is built using the Discrete Geometric Approach of Section 4.3.3. We observe that on simplicial meshes, the Discrete Geometric Approach leads to the same stiffness matrix as Whitney forms. We consider the first test case of the FVCA benchmark [24]. The domain $\Omega$ is the unit cube $[0,1]^{3}$, and the exact potential and the conductivity are

$$
p(x, y, z):=1+\sin (\pi x) \sin \left(\pi\left(y+\frac{1}{2}\right)\right) \sin \left(\pi\left(z+\frac{1}{3}\right)\right), \quad \underline{\lambda}:=\left[\begin{array}{ccc}
1 & 0.5 & 0 \\
0.5 & 1 & 0.5 \\
0 & 0.5 & 1
\end{array}\right] .
$$

The source term and the Dirichlet boundary condition are set according to (6.1). The integral of the source term is computed by a quadrature rule on the elementary subsimplices of each primal cell. Since the linear system is SPD by construction, a preconditioned Conjugate Gradient method is employed.

Two sequences of 3D polyhedral meshes are tested, each family consisting of successive uniform refinements of an initial mesh. The first mesh sequence contains prismatic meshes with polygonal basis, and the second checkerboard meshes. Examples are shown in Figure 7. Checkerboard meshes constitute a classical example of so-called non-matching meshes. For each mesh in both sequences, we compute three regularity criteria

$$
\Gamma_{1}:=\min _{c \in \mathrm{C}}\left(\frac{\underline{e} \cdot \underline{\tilde{f}}_{c}(e)}{|e|\left|\tilde{f}_{c}(e)\right|}\right), \quad \Gamma_{2}:=\min _{c \in \mathrm{C}}\left(\min _{e \in \mathrm{E}_{c}}\left(\frac{\left|\tilde{f}_{c}(e)\right|}{|e| h_{c}}, \frac{|e| h_{c}}{\left|\tilde{f}_{c}(e)\right|}\right)\right), \quad \Gamma_{3}:=\min _{v \in \mathrm{V}}\left(\frac{|\tilde{c}(v)|}{\sum_{e \in \mathrm{E}_{v}}|e||\tilde{f}(e)|}\right),
$$

the latter resulting from (3.13). $\Gamma_{1}$ evaluates how non-orthogonal the mesh is; $\Gamma_{1}=1$ means an orthogonal mesh. $\Gamma_{2}$ evaluates aspect ratios of primal and dual mesh entities. The smaller the value of the regularity criteria, the weaker the mesh regularity. 
TABLE 1. Results for prismatic meshes with polygonal basis.

\begin{tabular}{lccccccc}
\hline $\mathrm{n}_{\text {sys }}$ & stencil & ercl2 & rate & ener & rate & enerd & rate \\
\hline 1800 & 32 & $8.3 \mathrm{e}-03$ & & $1.7 \mathrm{e}-01$ & & $2.6 \mathrm{e}-02$ & \\
15200 & 35 & $2.3 \mathrm{e}-03$ & 1.8 & $8.6 \mathrm{e}-02$ & 0.9 & $8.0 \mathrm{e}-03$ & 1.7 \\
52200 & 37 & $1.0 \mathrm{e}-03$ & 1.9 & $5.8 \mathrm{e}-02$ & 1.0 & $3.9 \mathrm{e}-03$ & 1.7 \\
124800 & 37 & $6.0 \mathrm{e}-04$ & 1.9 & $4.4 \mathrm{e}-02$ & 1.0 & $2.4 \mathrm{e}-03$ & 1.7 \\
\hline
\end{tabular}

TABLE 2. Results for checkerboard meshes.

\begin{tabular}{lccccccc}
\hline $\mathrm{n}_{\text {sys }}$ & stencil & ercl2 & rate & ener & rate & enerd & rate \\
\hline 23 & 11 & $1.1 \mathrm{e}-01$ & & $5.2 \mathrm{e}-01$ & & $2.3 \mathrm{e}-01$ & \\
311 & 27 & $2.9 \mathrm{e}-02$ & 1.5 & $3.0 \mathrm{e}-01$ & 0.6 & $8.7 \mathrm{e}-02$ & 1.1 \\
3119 & 36 & $7.3 \mathrm{e}-03$ & 1.8 & $1.5 \mathrm{e}-01$ & 0.9 & $4.7 \mathrm{e}-02$ & 0.8 \\
27743 & 41 & $1.8 \mathrm{e}-03$ & 1.9 & $7.5 \mathrm{e}-02$ & 0.9 & $2.5 \mathrm{e}-02$ & 0.9 \\
233663 & 47 & $4.6 \mathrm{e}-04$ & 1.9 & $3.8 \mathrm{e}-02$ & 1.0 & $1.3 \mathrm{e}-02$ & 0.9 \\
\hline
\end{tabular}

For each mesh in both sequences, we report $\mathrm{n}_{\mathrm{sys}}$, the size of the linear system (for a vertex-based scheme, the number of primal vertices $\# \mathrm{~V}$ ), the (average) stencil of the scheme (the number of non-zero entries divided by the number of rows of the linear system) and the three following norms:

$$
\operatorname{ercl} 2:=\frac{\left\|p-L_{\mathcal{V}}^{\operatorname{conf}}(\mathbf{p})\right\|_{L^{2}(\Omega)}}{\|p\|_{L^{2}(\Omega)}}, \quad \text { ener }:=\frac{\left\|\underline{g}-\underline{L}_{\mathcal{E}}(\mathbf{g})\right\|_{\lambda}}{\|\underline{g}\|_{\lambda}}, \quad \text { enerd }:=\frac{\left\|\mathrm{R}_{\mathcal{E}}(\underline{g})-\mathbf{g}\right\|_{\lambda}}{\left\|\mathrm{R}_{\mathcal{E}}(\underline{g})\right\|_{\lambda}}
$$

where $p$ and $\underline{g}$ are the exact solution and gradient of (1.1), and $\mathbf{p}$ and $\mathbf{g}$ are the discrete potential and gradient resulting from (2.9). The potential reconstruction is that of Lemma 4.6. Rates of convergence between mesh $i$ and mesh $(i-1)$ of a mesh sequence are computed by rate $:=-3 \log \left(\frac{\operatorname{norm}(i)}{\operatorname{norm}(i-1)}\right) / \log \left(\frac{\mathrm{n}_{\mathrm{sys}}(i)}{\mathrm{n}_{\mathrm{sys}}(i-1)}\right)$.

Results are reported in Tables 1 and 2 for the prismatic and checkerboard mesh sequences, respectively. For the prismatic mesh sequence, the regularity criteria are $\Gamma_{1}=0.18, \Gamma_{2}=0.14$ and $\Gamma_{3}=0.0625$ (for any mesh in the sequence). The last linear system is solved in 144 iterations for a residual of $10^{-14}$. For the checkerboard mesh sequence, the regularity criteria are $\Gamma_{1}=0.89, \Gamma_{2}=0.14$ and $\Gamma_{3}=0.16$ (for any mesh in the sequence). The last linear system is solved in 128 iterations for a residual of $10^{-14}$. Concerning convergence rates, we observe that the results are in good agreement with the theoretical analysis. Indeed, we observe second-order convergence rate for the potential $L^{2}$-error and first-order convergence for the continuous and discrete energy errors (with some super-convergence for the discrete energy error in the case of prismatic meshes).

Remark 6.1 (Alternative potential reconstruction). Let $\mathrm{L}_{\mathcal{V}}^{1}(\mathbf{p})$ be piecewise affine on the submesh $\left\{\mathfrak{p}_{e, c}^{\mathrm{DGA}}\right\}_{e \in \mathrm{E}_{c}}$ defined in Section 4.3.3 with gradient given locally by $\left.\underline{\underline{L}}_{\mathcal{E}}(\mathbf{g})\right|_{\mathfrak{p}_{e} \mathrm{DA}}$ and fix the value $\mathrm{L}_{\mathcal{V}}^{1}(\mathbf{p})(v):=\mathbf{p}_{v}$ where $v$ is one of the endpoints of $e$ (the choice of $v$ is irrelevant). Then, our numerical experiments indicate that the difference $\left(p-\mathrm{L}_{\mathcal{V}}^{1}(\mathbf{p})\right)$ also converges to second-order in the $L^{2}$-norm.

\section{Conclusions}

In this work, we have analyzed Compatible Discrete Operator schemes for elliptic problems. We have considered both vertex-based and cell-based schemes. The cornerstone is the design of the discrete Hodge operator linking gradients to fluxes, and whose key properties have been stated first under an algebraic viewpoint and then using the idea of local (nonconforming) gradient reconstruction on a dual barycentric mesh. Links between the compatible discrete operator approach and various existing schemes have been explored. The present approach distinguishes the primal and dual meshes and handles the discrete gradient and divergence operators explicitly, without recombining them with other operators. In the vertex-based (resp., cell-based) setting, the discrete 
gradient is attached to the primal (resp., dual) mesh and the discrete divergence to the dual (resp., primal) mesh. This contrasts with Mimetic Finite Difference (either nodal or cell-based) and Finite Volume schemes which handle only one discrete differential operator explicitly (as already pointed out by Hiptmair [26]), and also with Discrete Duality Finite Volume schemes (see, e.g., Domelevo and Omnes [19] and Andreianov et al. [2]) which handle simultaneously the discrete gradient and divergence operators on both primal and dual meshes, thus leading to larger discrete systems. Additional topics to be explored regarding elliptic problems include hybridization of the cell-based scheme (in the spirit of hybrid Finite Volume schemes, see Eymard et al. [22]), higher-order extensions of both schemes (e.g., by allowing more than constants in the kernel of the relevant commuting operator), and extensive benchmarking to study the computational efficiency of the approach.

\section{Appendix A. Proof of Discrete Sobolev embedding}

Let a real number $1 \leq p<+\infty$. Let $\mathbf{p} \in \mathcal{V}$ and set $\mathbf{g}:=\operatorname{GRAD}(\mathbf{p}) \in \mathcal{E}$. We preliminarily observe that it suffices to prove the bound (3.18) for $q=p^{*}:=\frac{p d}{d-p}$, since the bounds for $1 \leq q<p^{*}$ then result from the bound for $q=p^{*}$ and the Hölder inequality (3.15).

The case $p=1$. Set $1^{*}=\frac{d}{d-1}$. A classical result (see, e.g., [22]) states that, for all $v \in L^{1}\left(\mathbb{R}^{d}\right)$ with bounded $\|\cdot\|_{\mathrm{BV}}$-norm, there holds $\|v\|_{L^{1^{*}}\left(\mathbb{R}^{d}\right)} \leq(2 d)^{-1}\|v\|_{\mathrm{BV}}$. Hence, recalling the operator $\mathrm{L}_{\mathcal{V}}^{0}$ introduced in Section 3.3.2 and using Theorem 3.4, we infer

$$
\left\|\mathrm{L}_{\mathcal{V}}^{0}(\mathbf{p})\right\|_{L^{1^{*}}\left(\mathbb{R}^{d}\right)} \leq(2 d)^{-1}\left\|\mathrm{~L}_{\mathcal{V}}^{0}(\mathbf{p})\right\|_{\mathrm{BV}} \leq(2 \sqrt{d})^{-1}\|\mathbf{g}\|_{1, \mathcal{E}}
$$

Since $\|\mathbf{p}\|_{1^{*}, \mathcal{V}}=\left\|\mathbf{L}_{\mathcal{V}}^{0}(\mathbf{p})\right\|_{L^{1^{*}}\left(\mathbb{R}^{d}\right)}$, we infer the desired bound (3.18) for $q=1^{*}$.

The case $1<p<d$. Let $\mu:=\frac{p(d-1)}{d-p}>1$ and observe that $p^{*}=\mu 1^{*}$. Let $|\mathbf{p}|^{\mu}$ denote the element of $\mathcal{V}$ whose components are, for all $v \in \mathrm{V},\left|\mathbf{p}_{v}\right|^{\mu}$. Applying the above bound to $|\mathbf{p}|^{\mu}$ yields

$$
\|\mathbf{p}\|_{p^{*}, \mathcal{V}}^{\mu}=\left\||\mathbf{p}|^{\mu}\right\|_{1^{*}, \mathcal{V}} \leq(2 \sqrt{d})^{-1}\left\|\operatorname{GRAD}\left(|\mathbf{p}|^{\mu}\right)\right\|_{1, \mathcal{E}}
$$

Let $e \in \mathrm{E}$ with vertices $v_{1}$ and $v_{2}$; since $\left|\operatorname{GRAD}\left(|\mathbf{p}|^{\mu}\right)\right|_{e} \leq \mu\left(\left|\mathbf{p}_{v_{1}}\right|^{\mu-1}+\left|\mathbf{p}_{v_{2}}\right|^{\mu-1}\right)\left|\mathbf{g}_{e}\right|$, we infer

$$
\left\|\operatorname{GRAD}\left(|\mathbf{p}|^{\mu}\right)\right\|_{1, \mathcal{E}} \leq \mu \sum_{c \in \mathrm{C}} \sum_{e \in \mathrm{E}_{c}} \sum_{v \in \mathrm{V}_{e}}\left|\tilde{f}_{c}(e)\|e\| \mathbf{p}_{v}\right|^{\mu-1}\left(\frac{\left|\mathbf{g}_{e}\right|}{|e|}\right)
$$

Using the Hölder inequality leads to

$$
\left\|\operatorname{GRAD}\left(|\mathbf{p}|^{\mu}\right)\right\|{ }_{1, \mathcal{E}} \leq \mu\left(\sum_{c \in \mathrm{C}} \sum_{e \in \mathrm{E}_{c}} \sum_{v \in \mathrm{V}_{e}}\left|\tilde{f}_{c}(e)\|e\| \mathbf{p}_{v}\right|^{p^{*}}\right)^{1 / \beta} 2^{1 / p}\|\mathbf{g}\|_{p, \mathcal{E}},
$$

with $\beta=\frac{p}{p-1}$ so that $\beta(\mu-1)=p^{*}$. Rearranging the order of summations and using (3.13) yields

$$
\sum_{c \in \mathrm{C}} \sum_{e \in \mathrm{E}_{c}} \sum_{v \in \mathrm{V}_{e}}\left|\tilde{f}_{c}(e)\|e\| \mathbf{p}_{v}\right|^{p^{*}} \leq \sum_{v \in \mathrm{V}} \sum_{e \in \mathrm{E}_{v}}\left|e\|\tilde{f}(e)\| \mathbf{p}_{v}\right|^{p^{*}} \leq \gamma^{-1}\|\mathbf{p}\|_{p^{*}, \mathcal{V}}^{p^{*}}
$$

Combining the above bounds with the fact that $\mu=\frac{p^{*}}{\beta}+1$ yields the desired bound (3.18) for $q=p^{*}$ with the constant $(2 \sqrt{d})^{-1} \mu 2^{1 / p} \gamma^{-1+1 / p}$.

The case $d \leq p$. Let $q_{1}>d$ and set $p_{1}:=\frac{d q_{1}}{d+q_{1}}$. There holds $1<p_{1}<d$ and $p_{1}^{*}=q_{1}$, so that $\|\mathbf{p}\|_{q_{1}, \mathcal{V}} \leq$ $C_{p_{1}, q_{1}}\|\mathbf{g}\|_{p_{1}, \mathcal{E}}$. Since $p_{1}<d \leq p$, the right-hand side can be bounded by $\|\mathbf{g}\|_{p, \mathcal{E}}$ owing to the inequality (3.15), whence the desired bound (3.18) for $q_{1}$. 


\section{Appendix B. Conforming Reconstructions}

\section{B.1. Proof of Lemma 4.6}

The conforming potential reconstruction is built as a piecewise affine function on a simplicial submesh of each primal cell, similarly to Christiansen [11] or to Brezzi et al. [8], but the present construction is different since additional properties related to geometric consistency must be fulfilled. Let $c \in \mathrm{C}$. For all $f \in \mathrm{F}_{c}$ and all $e \in \mathrm{E}_{f} \cap \mathrm{E}_{c}$, let $\mathfrak{p}_{e, f}:=\cup_{v \in \mathrm{V}_{e}} \mathfrak{s}\left(\underline{x}_{v}, \underline{x}_{e}, \underline{x}_{f}, \underline{x}_{c}\right) ; \mathfrak{p}_{e, f}$ is a simplex composed of two elementary subsimplices (see Fig. 6 right). Let $\mathfrak{S}_{c}$ be the simplicial submesh of $c$ composed of the simplices $\mathfrak{p}_{e, f}$. Let $\mathbf{p} \in \mathcal{V}_{c}$; then, $r_{\mathbf{p}}:=\mathrm{L}_{\mathcal{V}_{c}}^{\text {conf }}(\mathbf{p})$ is built in $\mathbb{P}_{1}\left(\mathfrak{S}_{c}\right)$ by prescribing its values on the vertices of $\mathfrak{S}_{c}$. Specifically, we set $r_{\mathbf{p}}\left(\underline{x}_{v}\right)=\mathbf{p}_{v}$ for all $v \in \mathrm{V}_{c}$, and prescribe $r_{\mathbf{p}}\left(\underline{x}_{f}\right)$ for all $f \in \mathrm{F}_{c}$ and $r_{\mathbf{p}}\left(\underline{x}_{c}\right)$ as follows:

$$
\begin{aligned}
& \sum_{v \in \mathrm{V}_{f}}|f \cap \tilde{c}(v)|\left(\mathbf{p}_{v}-r_{\mathbf{p}}\left(\underline{x}_{f}\right)\right)=0, \quad \forall f \in \mathrm{F}_{c}, \\
& \sum_{v \in \mathrm{V}_{c}}|c \cap \tilde{c}(v)|\left(\mathbf{p}_{v}-r_{\mathbf{p}}\left(\underline{x}_{c}\right)\right)=0 .
\end{aligned}
$$

Thus, $\mathrm{L}_{\mathcal{V}_{c}}^{\text {conf }}(\mathbf{p})=\sum_{v \in \mathrm{V}_{c}} \mathbf{p}_{v} \ell_{v, c}^{\text {conf }}$ for basis functions $\left\{\ell_{v, c}^{\text {conf }}\right\}_{v \in \mathrm{V}_{c}}$ satisfying $\ell_{v, c}^{\text {conf }}\left(v^{\prime}\right)=\delta_{v, v^{\prime}}$ for all $v^{\prime} \in \mathrm{V}_{c}$.

Lemma 4.6 (i) and (ii) (conformity and unisolvence) hold by construction. Lemma 4.6 (iii) (stability) can be proved using mesh regularity. Lemma 4.6 (iv) $\left(\mathbb{P}_{1}\right.$-consistency) results from the fact that the barycenters of the faces and of the cell satisfy $\sum_{v \in \mathrm{V}_{f}}|f \cap \tilde{c}(v)|\left(\underline{x}_{v}-\underline{x}_{f}\right)=0$ for all $f \in \mathrm{F}_{c}$ and $\sum_{v \in \mathrm{V}_{c}}|c \cap \tilde{c}(v)|\left(\underline{x}_{v}-\underline{x}_{c}\right)=0$ (this is where the fully barycentric subdivision is used). Finally, Lemma 4.6 (v) (geometric consistency) results from the fact that

$$
\int_{f} \ell_{v, c}^{\mathrm{conf}}=|f \cap \tilde{c}(v)|, \quad \int_{c} \ell_{v, c}^{\mathrm{conf}}=|c \cap \tilde{c}(v)| .
$$

These relations are verified directly by using vertex-based quadratures on the simplicial submesh. The identity $\int_{f} \ell_{v, c}^{\text {conf }}=|f \cap \tilde{c}(v)|$ then implies that (recall that $\underline{n}_{f, c}$ is the unit normal to $f$ pointing outward $c$ )

$$
\int_{c} \underline{\operatorname{grad}}\left(\ell_{v, c}^{\mathrm{conf}}\right)=\sum_{f \in \mathrm{F}_{v} \cap \mathrm{F}_{c}}|f \cap \tilde{c}(v)| \underline{n}_{f, c}=\sum_{e \in \mathrm{E}_{v} \cap \mathrm{E}_{c}} \iota_{v, e} \underline{\tilde{f}}_{c}(e),
$$

where we have used that the primal faces are planar. As a result, we infer, for all $\mathbf{p} \in \mathcal{V}, \int_{c} \operatorname{L}_{\mathcal{V}}^{\operatorname{conf}}(\mathbf{p})=$ $\sum_{v \in \mathrm{V}_{c}} \mathbf{p}_{v} \int_{c} \ell_{v, c}^{\mathrm{conf}}=\sum_{v \in \mathrm{V}_{c}} \mathbf{p}_{v}|c \cap \tilde{c}(v)|=\int_{c} \mathrm{~L}_{\mathcal{V}}^{0}(\mathbf{p})$, and

$$
\begin{aligned}
\int_{c} \underline{\operatorname{grad}}\left(\mathrm{L}_{\mathcal{V}}^{\text {conf }}(\mathbf{p})\right) & =\sum_{v \in \mathrm{V}_{c}} \mathbf{p}_{v} \int_{c} \underline{\operatorname{grad}}\left(\ell_{v, c}^{\text {conf }}\right)=\sum_{v \in \mathrm{V}_{c}} \mathbf{p}_{v}\left(\sum_{e \in \mathrm{E}_{v} \cap \mathrm{E}_{c}} \iota_{v, e} \underline{\tilde{f}}_{c}(e)\right) \\
& =\sum_{e \in \mathrm{E}_{c}}\left(\sum_{v \in \mathrm{V}_{e}} \iota_{v, e} \mathbf{p}_{v}\right) \underline{\tilde{f}}_{c}(e)=\sum_{e \in \mathrm{E}_{c}}(\operatorname{GRAD}(\mathbf{p}))_{e} \int_{c} \underline{\ell}_{e, c}=\int_{c} \underline{\mathrm{L}}_{\mathcal{E}}(\operatorname{GRAD}(\mathbf{p})),
\end{aligned}
$$

owing to the geometric consistency $(\mathbf{R} 4 \mathbf{v})$ of the reconstruction functions $\underline{\ell}_{e, c}$.

\section{B.2. Proof of Lemma 5.6}

We take inspiration from a construction by Vohralík and Wohlmuth [34]. Let $c \in$ C. For all $f \in \mathrm{F}_{c}$ and all $e \in \mathrm{E}_{f} \cap \mathrm{E}_{c}$, let $\mathfrak{p}_{e, f}$ be as in Section B.1 and define the triangle $\mathfrak{t}_{e, f}:=\mathfrak{p}_{e, f} \cap f$. Recalling $\mathfrak{S}_{c}$ from Section B.1, let $\mathbb{R} \mathbb{N}_{0}\left(\mathfrak{S}_{c}\right)$ be the lowest-order Raviart-Thomas-Nédélec finite element space on that submesh and let $\mathbb{P}_{0}^{*}\left(\mathfrak{S}_{c}\right)$ be spanned by piecewise constant functions with zero mean-value on $c$. For all $f \in \mathrm{F}_{c}$ and all $e \in \mathrm{E}_{f} \cap \mathrm{E}_{c}$, we build the function $\underline{\ell}_{e, f, c} \in \mathbb{R} \mathbb{T} \mathbb{N}_{0}\left(\mathfrak{S}_{c}\right)$ by solving the mixed finite element problem

$$
\begin{cases}\left(\underline{\ell}_{e, f, c}, \underline{\psi}_{h}\right)_{L^{2}(c)^{3}}-\left(\pi_{e, f, c}, \operatorname{div} \underline{\psi}_{h}\right)_{L^{2}(c)}=0 & \forall \underline{\psi}_{h} \in \mathbb{R T N}_{0,0}\left(\mathfrak{S}_{c}\right), \\ \left(\operatorname{div} \underline{\ell}_{e, f, c}, q_{h}\right)_{L^{2}(c)}=\left(d_{f, c}, q_{h}\right)_{L^{2}(c)}, & \forall q_{h} \in \mathbb{P}_{0}^{*}\left(\mathfrak{S}_{c}\right),\end{cases}
$$


with prescribed normal component $\int_{\mathfrak{t}_{f^{\prime}, e^{\prime}}} \underline{\ell}_{e, f, c} \cdot \underline{\nu}_{f^{\prime}}:=\delta_{e, e^{\prime}} \delta_{f, f^{\prime}}$ and prescribed (constant) divergence $d_{f, c}:=$ $|c|^{-1} \iota_{f, c}$ (observe that $\underline{\nu}_{f}$ is constant in $f$ since $f$ is planar). In $\left(\right.$ B.2), $\mathbb{R} \mathbb{T} \mathbb{N}_{0,0}\left(\mathfrak{S}_{c}\right)$ is the subspace of $\mathbb{R T N}_{0}\left(\mathfrak{S}_{c}\right)$ spanned by functions with zero normal component on $\partial c$, and $\pi_{e, f, c} \in \mathbb{P}_{0}^{*}\left(\mathfrak{S}_{c}\right)$ is the Lagrange multiplier. Then, for all $f \in \mathrm{F}_{c}$, we set

$$
\underline{\ell}_{f, c}^{\mathrm{conf}}:=|f|^{-1} \sum_{e \in \mathrm{E}_{f} \cap \mathrm{E}_{c}}\left|\mathfrak{t}_{e, f}\right| \underline{\ell}_{e, f, c},
$$

and, for all $\phi \in \mathcal{F}_{c}, \underline{\underline{L}}_{\mathcal{F}_{c}}^{\text {conf }}(\phi):=\sum_{f \in \mathrm{F}_{c}} \phi_{f} \underline{\ell}_{f, c}^{\text {conf }}$. Finally, $\underline{\underline{L}}_{\mathcal{F}}^{\text {conf }}$ is assembled elementwise from $\underline{\mathrm{L}}_{\mathcal{F}_{c}}^{\text {conf }}$.

Lemma 5.6 (i) and (ii) are readily verified. Turning to point (v), it suffices to show that $\int_{c} \underline{\ell}_{f, c}^{\text {conf }}=\underline{\tilde{e}}_{c}(f)$. Let $\underline{G} \in \mathbb{R}^{3}$. Since $\operatorname{div}\left(\underline{\ell}_{e, f, c}\right)$ is constant in $c$ and $\underline{x}_{c}$ is the barycenter of $c$ (fully barycentric subdivision), integration by parts yields

$$
\int_{c} \underline{\ell}_{e, f, c} \cdot \underline{G}=\int_{c} \underline{\ell}_{e, f, c} \cdot \underline{\operatorname{grad}}\left(\underline{G} \cdot\left(\underline{x}-\underline{x}_{c}\right)\right)=\sum_{f^{\prime} \in \mathrm{F}_{c}} \int_{f^{\prime}}\left(\underline{\ell}_{e, f, c} \cdot \underline{\nu}_{c}\right)\left(\underline{G} \cdot\left(\underline{x}-\underline{x}_{c}\right)\right)=\iota_{f, c} \underline{G} \cdot\left(\underline{x}_{e, f}-\underline{x}_{c}\right),
$$

where $\underline{x}_{e, f}$ is the barycenter of $\mathfrak{t}_{e, f}$. Summing over $e \in \mathrm{E}_{f} \cap \mathrm{E}_{c}$ using (B.3) yields the desired result since $\sum_{e \in \mathrm{E}_{f} \cap \mathrm{E}_{c}}\left|\mathfrak{t}_{e, f}\right| \underline{x}_{e, f}=|f| \underline{x}_{f}$. Let now $\phi \in \mathcal{F}_{c}$. Then, by linearity, $\underline{r}_{\phi}:=\underline{\mathfrak{L}}_{\mathcal{F}_{c}}^{\text {conf }}(\phi)$ is in $\mathbb{R} \mathbb{T} \mathbb{N}_{0}\left(\mathfrak{S}_{c}\right)$ and solves

$$
\begin{cases}\left(\underline{r}_{\phi}, \underline{\psi}_{h}\right)_{L^{2}(c)^{3}}-\left(\pi_{\boldsymbol{\phi}}, \operatorname{div} \underline{\psi}_{h}\right)_{L^{2}(c)}=0 & \forall \underline{\psi}_{h} \in \mathbb{R} \mathbb{T N}_{0,0}\left(\mathfrak{S}_{c}\right), \\ \left(\operatorname{div} \underline{\underline{r}}_{\boldsymbol{\phi}}, q_{h}\right)_{L^{2}(c)}=\left(d_{\boldsymbol{\phi}}, q_{h}\right)_{L^{2}(c)}, & \forall q_{h} \in \mathbb{P}_{0}^{*}\left(\mathfrak{S}_{c}\right),\end{cases}
$$

with prescribed normal component $\int_{\mathfrak{t}_{f, e}} \underline{r}_{\phi} \cdot \underline{\nu}_{f}:=\phi_{f}\left(\left|\mathfrak{t}_{e, f}\right| /|f|\right)$ and prescribed (constant) divergence $d_{\phi}:=$ $|c|^{-1} \sum_{f \in \mathrm{F}_{c}} \iota_{f, c} \boldsymbol{\phi}_{f}$. Classical stability estimates yield $\left\|\underline{r}_{\phi}\right\|_{L^{2}(c)^{3}} \lesssim\|\phi\|_{2, \mathcal{F}_{c}}$ and, hence, point (iii). Finally, to prove point (iv), it suffices to show that, if $\underline{\Phi}$ is constant in $c$, letting $\phi:=\mathrm{R}_{\mathcal{F}_{c}}(\underline{\Phi})$, there holds $\underline{r}_{\phi}=\underline{\Phi}$. First, using point (v) and the property of dual barycentric meshes that $\sum_{f \in \mathrm{F}_{c}} \underline{\tilde{e}}_{c}(f) \otimes \underline{f}=|c| \underline{\underline{\mathrm{d}}}$, we infer $|c|^{-1} \int_{c} \underline{r}_{\phi}=\underline{\Phi}$ so that $\|\underline{\Phi}\|_{L^{2}(c)^{3}} \leq\left\|\underline{r}_{\phi}\right\|_{L^{2}(c)^{3}}$. However, $\underline{\Phi}$ is in $\mathbb{R T N}_{0}\left(\mathfrak{S}_{c}\right)$ and satisfies the prescriptions on the normal component and (constant) divergence. Since $\underline{\underline{r}}_{\phi}$ minimizes the $L^{2}$-norm in this space under these constraints, we infer $\underline{r}_{\phi}=\underline{\Phi}$. The proof is complete.

Acknowledgements. The authors are thankful to Robert Eymard (University Marne-la-Vallée) and Martin Vohralík (INRIA Rocquencourt) for stimulating discussions.

\section{REFERENCES}

[1] C. Amrouche, C. Bernardi, M. Dauge and V. Girault, Vector potentials in three-dimensional non-smooth domains. Math. Meth. Appl. Sci. 21 (1998) 823-864.

[2] B. Andreianov, F. Boyer and F. Hubert, Discrete duality finite volume schemes for Leray-Lions-type elliptic problems on general 2D meshes. Numer. Methods Partial Differ. Eqs. 23 (2007) 145-195.

[3] D.N. Arnold, R.S. Falk and R. Winther, Finite element exterior calculus, homological techniques, and applications. Acta Numerica 15 (2006) 1-155.

[4] A. Back, Étude théorique et numérique des équations de Vlasov-Maxwell dans le formalisme covariant. Ph.D. thesis, University of Strasbourg (2011).

[5] P. Bochev and J.M. Hyman, Principles of mimetic discretizations of differential operators, Compatible Spatial Discretization. In vol. 142 of The IMA Volumes Math. Appl., edited by D. Arnold, P. Bochev, R. Lehoucq, R.A. Nicolaides and M. Shashkov (2005) 89-120.

[6] A. Bossavit, On the geometry of electromagnetism. J. Japan Soc. Appl. Electromagn. Mech. 6 (1998) (no 1) 17-28, (no 2) 114-23, (no 3) 233-40, (no 4) 318-26.

[7] A. Bossavit, Computational electromagnetism and geometry. J. Japan Soc. Appl. Electromagn. Mech. 7-8 (1999-2000) (no 1) 150-9, (no 2) 294-301, (no 3) 401-8, (no 4) 102-9, (no 5) 203-9, (no 6) 372-7.

[8] F. Brezzi, A. Buffa and K. Lipnikov, Mimetic finite difference for elliptic problem. Math. Model. Numer. Anal. 43 (2009) $277-295$.

[9] F. Brezzi, K. Lipnikov and M. Shashkov, Convergence of the mimetic finite difference method for diffusion problems on polyhedral meshes. SIAM J. Numer. Anal. 43 (2005) 1872-1896. 
[10] A. Buffa and S.H. Christiansen, A dual finite element complex on the barycentric refinement. Math. Comput. 76 (2007) 1743-1769.

[11] S.H. Christiansen, A construction of spaces of compatible differential forms on cellular complexes. Math. Models Methods Appl. Sci. 18 (2008) 739-757.

[12] S.H. Christiansen, H.Z. Munthe-Kaas and B. Owren, Topics in structure-preserving discretization. Acta Numer. 20 (2011) $1-119$.

[13] L. Codecasa, R. Specogna and F. Trevisan, Base functions and discrete constitutive relations for staggered polyhedral grids. Comput. Methods Appl. Mech. Engrg. 198 (2009) 1117-1123.

[14] L. Codecasa, R. Specogna and F. Trevisan, A new set of basis functions for the discrete geometric approach. J. Comput. Phys. 229 (2010) 7401-7410.

[15] L. Codecasa and F. Trevisan, Convergence of electromagnetic problems modelled by discrete geometric approach. CMES $\mathbf{5 8}$ (2010) 15-44.

[16] M. Desbrun, A.N. Hirani, M. Leok and J.E. Marsden, Discrete Exterior Calculus. Technical report (2005).

[17] D.A. Di Pietro and A. Ern, Mathematical Aspects of Discontinuous Galerkin Methods, in vol. 69 of SMAI Math. Appl. Springer (2012).

[18] J. Dodziuk, Finite-difference approach to the Hodge theory of harmonic forms. Amer. J. Math. 98 (1976) 79-104.

[19] K. Domelevo and P. Omnes, A finite volume method for the Laplace equation on almost arbitrary two-dimensional grids. ESAIM: M2AN 39 (2005) 1203-1249.

[20] J. Droniou and R. Eymard, A mixed finite volume scheme for anisotropic diffusion problems on any grid. Numer. Math. 105 (2006) 35-71.

[21] J. Droniou, R. Eymard, T. Gallouët and R. Herbin, A Unified Approach to Mimetic Finite Difference, Hybrid Finite Volume and Mixed Finite Volume Methods. Math. Models and Methods Appl. Sci. 20 (2010) 265-295.

[22] R. Eymard, T. Gallouët and R. Herbin, Discretization of heterogeneous and anisotropic diffusion problems on general nonconforming meshes SUSHI: a scheme using stabilization and hybrid interfaces. IMA J. Numer. Anal. 30 (2010) 1009-1043.

[23] R. Eymard, C. Guichard and R. Herbin, Small stencil 3d schemes for diffusive flows in porious media. ESAIM: M2AN 46 (2012) 265-290.

[24] R. Eymard, G. Henry, R. Herbin, F. Hubert, R. Klöfkorn and G. Manzini, 3d benchmark on discretization schemes for anisotropic diffusion problems on general grids, in vol. 2 of Finite Volumes for Complex Applic. VI - Problems Perspectives. Springer (2011) 95-130.

[25] A. Gillette, Stability of dual discretization methods for partial differential equations. Ph.D. thesis, University of Texas at Austin (2011).

[26] R. Hiptmair, Discrete hodge operators: An algebraic perspective. Progress In Electromagnetics Research 32 (2001) 247-269.

[27] Xiao Hua Hu and R.A. Nicolaides, Covolume techniques for anisotropic media. Numer. Math. 61 (1992) $215-234$.

[28] J. Hyman and J. Scovel, Deriving mimetic difference approximations to differential operators using algebraic topology. Los Alamos National Laboratory (1988).

[29] J. Kreeft, A. Palha and M. Gerritsma, Mimetic framework on curvilinear quadrilaterals of arbitrary order. Technical Report, Delft University (2011) ArXiv: 1111.4304v1.

[30] C. Mattiussi, The finite volume, finite element, and finite difference methods as numerical methods for physical field problems. In vol. 113 of Advances in Imaging and Electron Phys. Elsevier (2000) 1-146.

[31] J.B. Perot and V. Subramanian, Discrete calculus methods for diffusion. J. Comput. Phys. 224 (2007) 59-81.

[32] T. Tarhasaari, L. Kettunen and A. Bossavit, Some realizations of a discrete hodge operator: A reinterpretation of finite element techniques. IEEE Transactions on Magnetics 35 (1999) 1494-1497.

[33] E. Tonti, On the formal structure of physical theories. Instituto di matematica, Politecnico, Milano (1975).

[34] M. Vohralík and B. Wohlmuth, Mixed finite element methods: implementation with one unknown per element, local flux expressions, positivity, polygonal meshes, and relations to other methods. Math. Models Methods Appl. Sci. 23 (2013) 803838.

[35] H. Whitney, Geometric integration theory. Princeton University Press, Princeton, N.J. (1957).

[36] S. Zaglmayr, High order finite element methods for electromagnetic field computation. Ph.D. thesis, Johannes Kepler Universität Linz (2006). 